\title{
Characterizing K2 Candidate Planetary Systems Orbiting Low-mass Stars. II. Planetary Systems Observed During Campaigns 1-7
}

Courtney D. Dressing ${ }^{1,2,15}$ (1) Andrew Vanderburg $^{3,16}$ (1) , Joshua E. Schlieder ${ }^{4,5}$ (i), Ian J. M. Crossfield ${ }^{6,7,15}$, Heather A. Knutson ${ }^{1}$, Elisabeth R. Newton ${ }^{7,17}$ (1), David R. Ciardi ${ }^{4}$, Benjamin J. Fulton ${ }^{8,9}$ (i), Erica J. Gonzales ${ }^{10,16,18}$, Andrew W. Howard ${ }^{9}$ (i),

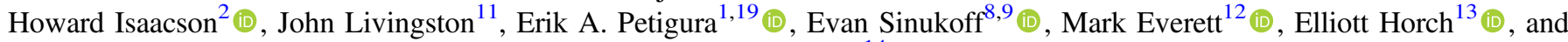
Steve B. Howell ${ }^{14}$ (1)

${ }^{1}$ Division of Geological \& Planetary Sciences, California Institute of Technology, Pasadena, CA 91125, USA; dressing @ berkeley.edu

${ }_{3}^{2}$ Astronomy Department, University of California, Berkeley, CA 94720, USA

${ }^{3}$ Harvard-Smithsonian Center for Astrophysics, Cambridge, MA 02138, USA

${ }^{4}$ IPAC-NExScI, Mail Code 100-22, Caltech, 1200 E. California Boulevard, Pasadena, CA 91125, USA

${ }^{5}$ NASA Goddard Space Flight Center, Greenbelt, MD 20771, USA

${ }^{6}$ Department of Astronomy and Astrophysics, University of California, Santa Cruz, CA 95064, USA

7 Department of Physics, Massachusetts Institute of Technology, Cambridge, MA 02139, USA

${ }^{8}$ Institute for Astronomy, University of Hawai'i at Mānoa, Honolulu, HI 96822, USA

${ }^{9}$ Cahill Center for Astrophysics, California Institute of Technology, Pasadena, CA 91125, USA

${ }^{10}$ Department of Physics, University of Notre Dame, Notre Dame, IN 46556, USA

${ }^{11}$ The University of Tokyo, 7-3-1 Bunkyo-ku, Tokyo 113-0033, Japan

${ }^{12}$ National Optical Astronomy Observatory, 950 North Cherry Avenue, Tucson, AZ 85719, USA

${ }^{13}$ Department of Physics, Southern Connecticut State University, 501 Crescent Street, New Haven, CT 06515, USA

${ }^{14}$ Space Science and Astrobiology Division, NASA Ames Research Center, Moffett Field, CA 94035, USA

Received 2017 March 21; revised 2017 August 8; accepted 2017 August 24; published 2017 November 3

\begin{abstract}
We recently used near-infrared spectroscopy to improve the characterization of 76 low-mass stars around which K2 had detected 79 candidate transiting planets. 29 of these worlds were new discoveries that had not previously been published. We calculate the false positive probabilities that the transit-like signals are actually caused by nonplanetary astrophysical phenomena and reject five new transit-like events and three previously reported events as false positives. We also statistically validate 17 planets ( 7 of which were previously unpublished), confirm the earlier validation of 22 planets, and announce 17 newly discovered planet candidates. Revising the properties of the associated planet candidates based on the updated host star characteristics and refitting the transit photometry, we find that our sample contains 21 planets or planet candidates with radii smaller than $1.25 R_{\oplus}, 18$ super-Earths $\left(1.25-2 R_{\oplus}\right), 21$ small Neptunes $\left(2-4 R_{\oplus}\right)$, three large Neptunes $\left(4-6 R_{\oplus}\right)$, and eight giant planets $\left(>6 R_{\oplus}\right)$. Most of these planets are highly irradiated, but EPIC 206209135.04 (K2-72e, $1.29_{-0.13}^{+0.14} R_{\oplus}$ ), EPIC 211988320.01 $\left(R_{p}=2.86_{-0.15}^{+0.16} R_{\oplus}\right)$, and EPIC $212690867.01\left(2.20_{-0.18}^{+0.19} R_{\oplus}\right)$ orbit within optimistic habitable zone boundaries set by the "recent Venus" inner limit and the "early Mars" outer limit. In total, our planet sample includes eight moderately irradiated $1.5-3 R_{\oplus}$ planet candidates $\left(F_{p} \lesssim 20 F_{\oplus}\right)$ orbiting brighter stars $(K s<11)$ that are well-suited for atmospheric investigations with the Hubble, Spitzer, and/or James Webb Space Telescopes. Five validated planets orbit relatively bright stars $(K p<12.5)$ and are expected to yield radial velocity semi-amplitudes of at least $2 \mathrm{~m} \mathrm{~s}^{-1}$. Accordingly, they are possible targets for radial velocity mass measurement with current facilities or the upcoming generation of red optical and near-infrared high-precision RV spectrographs.
\end{abstract}

Key words: planetary systems - planets and satellites: fundamental parameters - stars: fundamental parameters stars: late-type - stars: low-mass - techniques: spectroscopic

Supporting material: machine-readable table

\section{Introduction}

Since 2014, the NASA K2 mission has been using the Kepler spacecraft to search for transiting planets in 100 sq. deg. fields along the ecliptic plane. K2 observes 10,000-30,000 stars in each field for roughly 80 days before moving onto the next field. The placement of each field is driven by the three primary Requirements of the extended mission design: (1) K2

\footnotetext{
15 NASA Sagan Fellow.

16 National Science Foundation Graduate Research Fellow.

17 National Science Foundation Astronomy \& Astrophysics Postdoctoral Fellow.

18 Deans' Graduate Fellow.

19 NASA Hubble Fellow.
}

must look along the ecliptic plane so that the torque from solar radiation pressure is balanced, (2) sunlight must illuminate the solar panels, and (3) K2 cannot look so close to the Sun that sunlight illuminates the detectors (Howell et al. 2014; Van Cleve et al. 2016).

During the main Kepler mission, the planet search targets were primarily selected by the Kepler Science Office with a small contribution from Guest Observer proposals. In contrast, all K2 targets are nominated by members of the community. Although some of the selected target stars are well-characterized, many have poorly estimated properties constrained by only photometry, proper motion, and (when lucky) parallax. The problem of inadequate stellar characterization is particularly dire for the smallest, coolest target stars around which K2 
has the highest sensitivity to transiting planets. In order to improve the characterization of low-mass K2 target stars, ${ }^{20}$ we are conducting an extensive spectroscopic survey of potentially low-mass stars. In the first paper in this series (Dressing et al. 2017, hereafter D17), we presented near-infrared (NIR) spectra and determined stellar parameters for 144 potentially low-mass stars observed by K2 during Campaigns 0-7 (2014 May 30-2015 December 26).

In this paper, we use our previously determined stellar parameters combined with planet candidate lists to generate a catalog of K2 planetary systems orbiting low-mass stars. The structure of the paper is as follows.

In Section 2, we explain the origins of our planet candidate sample and describe the $\mathrm{K} 2$ planet candidate lists from which we selected our targets. We then consult the catalog of stellar parameters presented in D17 and update the host star parameters accordingly in Section 3. Next, we fit the K2 photometry in Section 4 to determine the transit parameters for each candidate. In Section 5, we use the open-source VESPA software (Morton 2015b) to run a false positive analysis to statistically validate planets and identify likely false positives. In Section 6, we combine the information from our various analyses to revise parameters for $\mathrm{K} 2$ planet candidates and validated planets orbiting low-mass dwarfs. We highlight particularly noteworthy individual systems in Section 7 before concluding in Section 8.

\section{Sources of Planet Candidates}

We obtained NIR spectroscopy of all stars in our sample because they were initially believed to host transiting planets or because they were close enough to the candidate host star that they might have been responsible for the transit-like signal observed in the light curve. In some cases, subsequent detailed analyses of the K2 photometry or ground-based follow-up observations revealed that the putative transit signals were actually due to false positives.

The majority of the 74 systems in the cool dwarf sample were selected from unpublished candidate lists provided by A. Vanderburg (53 stars hosting 59 candidates) or the K2 California Consortium (K2C2; 43 stars hosting 54 candidates); several stars appear on both lists and many of the $\mathrm{K} 2 \mathrm{C} 2$ targets were later published in Crossfield et al. (2016). The cool dwarf sample also includes 20 single-planet systems from Barros et al. (2016), 16 singles from Pope et al. (2016), 16 stars hosting 18 candidates reported by Vanderburg et al. (2016b), three singles from Adams et al. (2016), two singles from Mann et al. (2017), and five singles from Montet et al. (2015), who refined the properties of the planet candidates reported by Foreman-Mackey et al. (2015). Two of the stars in the sample (EPIC 212773309 and EPIC 211694226) are in close proximity to other stars and EPIC 212773309 also displays a clear secondary eclipse.

The 74 systems in our cool dwarf sample host 79 unique K2 Objects of Interest (K2OIs). As of 2016 September 7, 24 of those K2OIs had been confirmed as bona fide planets using the VESPA false positive probability tool (Morton 2012, 2015b, see Section 5), 23 had been previously published as planet candidates, two were classified as false positives, and 30 were new detections. Twenty seven of the new detections were identified by A. Vanderburg,

\footnotetext{
${ }^{20}$ In this series we define "low-mass stars" as $\mathrm{M}$ and $\mathrm{K}$ dwarfs with $M_{\star} \lesssim 0.75 M_{\odot}$.
}

eight were found by $\mathrm{K} 2 \mathrm{C} 2$, and five were discovered by both collaborations. In total, our cool dwarf planet sample consists of 60 single-planet systems, six double-planet systems $\quad($ EPIC $201549860=\mathrm{K} 2-35, \quad$ EPIC $206011691=\mathrm{K} 2-$ 21, EPIC $210508766=\mathrm{K} 2-83$, EPIC $210968143=\mathrm{K} 2-90$, EPIC 211305568, and EPIC 211331236 = K2-117), one tripleplanet system (EPIC 211428897) and one quadruple-planet system (EPIC $206209135=\mathrm{K} 2-72$ ).

\section{Updates to Planet Host Star Characterization}

In D17, we applied empirical relations to NIR spectra acquired at IRTF/SpeX and Palomar/TSPEC to revise the classifications of putative low-mass stars harboring potential K2 planet candidates. Of the $144 \mathrm{~K} 2$ targets we observed, $49 \%$ were actually contaminating giant stars or hotter dwarfs (typically reddened by interstellar extinction) and $74(51 \%)$ were bona fide low-mass dwarfs. For the cool dwarfs, we measured a series of equivalent widths and spectral indices and applied empirically based relations from Newton et al. (2015) and Mann et al. (2013a, 2015) to estimate effective temperatures, radii, masses, metallicities, and luminosities. Our results agree well with those of Martinez et al. (2017), who used lower-resolution NIR spectra from NTT/SOFI to improve the characterization of late-type dwarfs hosting $\mathrm{K} 2$ planet candidates.

In general, we found that our new radii were typically $0.13 R_{\odot}(39 \%)$ larger than the original estimates provided in the Ecliptic Plane Input Catalog (EPIC, Huber et al. 2016). These changes are unsurprising because, as noted by Huber et al. (2016), the EPIC values were determined by comparing photometry to stellar models that have been shown to systematically underestimate the temperatures and radii of cool stars (e.g., Kraus et al. 2011; Boyajian et al. 2012; Feiden \& Chaboyer 2012; Spada et al. 2013; von Braun et al. 2014; Newton et al. 2015). In addition, our quest to find potentially habitable planets likely biased our stellar sample toward stars with underestimated radii at the expense of stars with overestimated radii. We adopt the revised stellar classifications from D17 in this paper.

Although the planet candidate catalogs we consulted typically provided their own estimates of stellar properties, we found that the original stellar classifications provided therein also tended to underestimate the radii and temperatures of the cool dwarfs. For most candidates, the amplitude of the suggested radius change is similar to the $15 \%$ radius increase found by Newton et al. (2015) for Kepler planet candidates orbiting $\mathrm{M}$ dwarfs with previous radius estimates based on fits to stellar models (e.g., Muirhead et al. 2012, 2014; Dressing \& Charbonneau 2013; Mann et al. 2013b; Huber et al. 2014).

The exceptions to the general trend of underestimated stellar radii are the parameters provided in Vanderburg et al. (2016b) and the associated unpublished Vanderburg lists. Indeed, their estimates are based on empirical relations (Casagrande et al. 2008; González Hernández \& Bonifacio 2009; Boyajian et al. 2013; Pecaut \& Mamajek 2013). For those two sources, the discrepancies between our revised values and their initial values are likely due to the fact that spectroscopic observations help break the degeneracy between stars that are intrinsically red and stars that appear red due to interstellar extinction. 


\section{Improving Transit Fits}

Some of the planets in our target list have well-determined properties because they were previously published in other catalogs, but others are new. In order to provide a uniform catalog of properties for all of the planets in our sample, we perform our own fits to the $\mathrm{K} 2$ photometry to determine updated properties and errors for the full planet sample.

We began by downloading the K2 Self Flat Fielding (K2SFF) photometry provided by A. Vanderburg. ${ }^{21}$ The K2SFF pipeline processes $\mathrm{K} 2$ photometry by recording the roll angle of the spacecraft during each cadence and removing the correlation between flux and roll angle (Vanderburg \& Johnson 2014; Vanderburg et al. 2016b). This procedure yields precision within $60 \%$ of that achieved during the baseline Kepler mission for faint stars $(K p>12.5)$ and Kepler-like performance for brighter stars. Prior to fitting the transits, we re-processed the K2SFF data by simultaneously fitting for the transit light curves, long-term variability, and $\mathrm{K} 2$ pointing systematics using the procedure described by Vanderburg et al. (2016b).

Next, we ran a Markov chain Monte Carlo (MCMC) analysis to constrain the time of transit $T_{0}$, orbital period $P$, planet/star radius ratio $R_{p} / R_{*}$, semimajor axis/stellar radius ratio $a / R_{*}$, inclination $i$, quadratic limb darkening, eccentricity $e$, and longitude of periastron $\omega$. We ran our fits in Python and used the emcee package (Foreman-Mackey et al. 2013) to determine the errors on transit parameters. We assessed convergence by measuring the integrated autocorrelation time of our chains and requiring that the MCMC ran for at least ten times longer than the estimated autocorrelation time.

In order to efficiently sample the full allowed parameter space, we fit the limb darkening parameters using the $q_{1}, q_{2}$ coordinatespace defined by Kipping (2013a). We also assumed that the eccentricity distribution of transiting planets followed the Beta distribution found by Kipping (2013b) for short-period planets $\left(P<382\right.$ days, $P_{\beta}(e ; \alpha, \beta)=\frac{\Gamma(\alpha+\beta)}{\Gamma(\alpha) \Gamma(\beta)} e^{\alpha-1}(1-e)^{\beta-1}$ where $\alpha=0.697, \beta=3.27$, and $\Gamma$ is the Gamma function) and fit for $\sqrt{e} \cos \omega$ and $\sqrt{e} \sin \omega$ to enable more efficient sampling of loweccentricity orbits (e.g., Eastman et al. 2013).

At each point in the analysis, we computed the likelihood of our transit model by using the BATMAN package written by Kreidberg (2015) to solve the equations of Mandel \& Agol (2002) and generate a model transit lightcurve. Our K2 light curves were obtained in "long-cadence" mode using $30 \mathrm{~min}$ integration times, which is relatively long compared to total durations of the transits we model. Accordingly, we employed the "supersample" feature of BATMAN to generate sample long-cadence light curves by modeling the brightness of the star at $1 \mathrm{~min}$ cadence and recording the average of 30 consecutive modeled fluxes.

We restricted our fits to consider $70^{\circ}<i<90^{\circ}, 0<R_{p} / R_{*}$ $<1, a / R_{*}>1,0 \leqslant q_{1} \leqslant 1,0 \leqslant q_{2} \leqslant 1,0<\sqrt{e} \cos \omega<1$, and $0<\sqrt{e} \sin \omega<1$. We further required that K2OIs transit their host stars (i.e., impact parameter $b \leqslant 1+R_{P} / R_{\star}$ ) and imposed priors on the limb-darkening coefficients by interpolating the tables produced by Claret et al. (2012) at the temperatures and surface gravities of the host stars. ${ }^{22}$ Specifically, we assumed that $u_{1}$ and $u_{2}$ were drawn from Gaussian distributions with dispersions set by propagating the errors in our stellar parameter

\footnotetext{
21 https://archive.stsci.edu/prepds/k2sff/; https://doi.org/10.17909/T9BC75

${ }^{22}$ Claret et al. (2012) consider multiple methods for computing limbdarkening coefficients; we adopt the coefficients found using the least-squares method.
}

estimates. Despite our attention to limb darkening, we note that the dominant contribution to the shape of ingress and egress is the smearing due to the lengthy exposure times used for long-cadence $K 2$ data.

All of our target stars were spectroscopically characterized by D17. We incorporated our knowledge of the host stars into our transit fits by using Kepler's third law and the estimated host star masses to determine the orbital semimajor axes of planets with the observed orbital periods. We then compared the resulting distances to the estimated stellar radii and set Gaussian priors on the expected $a / R_{*}$ ratio for each planet (Seager \& Mallén-Ornelas 2003; Sozzetti et al. 2007; Torres et al. 2008). The widths of these priors reflect the uncertainties in the stellar radii and masses, ${ }^{23}$ but by imposing this prior we implicitly assume that the candidate transit event is indeed caused by the transit of a planet across the target star rather than a blended transit or eclipse of a contaminating star. The VESPA false positive analysis discussed in Section 5 should reveal such scenarios.

For a handful of targets, the transit depths were shallow enough that the MCMC sometimes wandered away from the transit signal under consideration. Accordingly, we required that the transit center must be within $6 \%$ of the initial guess (up to a maximum difference of $6 \mathrm{hr}$ ) and that the orbital period must be within 0.025 days $(36 \mathrm{~min})$ of the initial guess. As discussed in Section 6.3, we later repeated the transit fitting process using k2phot and $\mathrm{K} 2 \mathrm{SC}$ photometry to check for systematic offsets in planet parameters.

\section{Determining K2OI Dispositions}

Early in the Kepler mission, transiting planet candidates were "confirmed" by conducting radial velocity or transit timing variation studies to measure planet masses. However, mass measurements are expensive and not feasible for all systems. As a result, tools like BLENDER (e.g., Fressin et al. 2011; Torres et al. 2011), VESPA (Morton 2012, 2015b), and PASTIS (Díaz et al. 2014) have been adopted to "validate" planet candidates in a statistical sense. These tools simulate the range of astrophysical configurations that could generate the observed lightcurve subject to the constraints placed by indepth analyses of the transit photometry (e.g., shifts in the photocenter during transit, presence or absence of secondary eclipse, variations in the depths of odd and even transits) and subsequent follow-up investigations (e.g., high-contrast imaging, radial velocity searches for additional stars, achromaticity of transit events, analyses of archival observations).

\subsection{Identifying Clear False Positives}

Four of the K2OIs in our sample displayed clear secondary eclipses when we phase-folded the light curve of the host star to the assumed orbital period: EPIC 212679798.01, 212773272.01, 212773309.01 (also identified as an EB by Barros et al. 2016), and 213951550.01. Consulting the

\footnotetext{
${ }^{23}$ As discussed in D17, we estimated the stellar radius errors by running Monte Carlo simulations in which we simulated multiple realizations of the noise in our NIR spectra and recomputed the stellar parameters using the H-band relations from Newton et al. (2015). Our radius errors include a $0.027 R_{\odot}$ intrinsic scatter term added in quadrature to the $1 \sigma$ errors determined from the Monte Carlo simulations. We determined the masses and associated errors by employing the stellar effective temperature-mass relation from Mann et al. (2013b). In this paper, we enforce a minimum mass error of $10 \%$ based on the fractional errors displayed in Figure 5 of Mann et al. (2013b).
} 
ExoFOP-K2 follow-up website, ${ }^{24}$ we found that three of these stars were flagged by $\mathrm{D}$. LaCourse as possible eclipsing binaries (212773309 and 212679798) or false positives (212773272). K2OIs 212773309.01 and 212773272.01 were identified at exactly the same ephemeris, suggesting that the transit-like events detected in the light curve of EPIC 212773272 are actually due to the eclipses of EPIC 212773309.

In addition to classifying EPIC 212679798.01, 2127732 $72.01,212773309.01$, and 213951550.01 as false positives due to the presence of secondary eclipses, we also rejected EPIC 211831378.01, 211970234.01, and 212572452.01 due to blended photometry or inconsistent transit depths when fitting data processed by different $\mathrm{K} 2$ pipelines. For instance, the K2SFF photometry of EPIC 211831378 displays 650 ppm transits while that from k2phot and K2SC reveals events with depths of $9000 \mathrm{ppm}$ and $12 \%$, respectively. We attribute this discrepancy in event depth to the use of different apertures for each pipeline. As mentioned on the ExoFOP website, archival photometry from DSS, SDSS, 2MASS, and WISE reveals a brighter star 12". 3 away from EPIC 211831378; the K2SFF pipeline incorrectly placed the aperture around this star (EPIC 211831539) rather than around the target star.

EPIC 211970234 is also in a crowded field and the assigned K2SFF aperture includes contributions from multiple stars, one of which is much brighter than the target star. Similarly, the photometry of EPIC 212572452 is contaminated by light from EPIC 212572439 (2MASS J13374562-1111331), which is 1 magnitude brighter than the target star and 5".96 away.

Finally, we classified EPIC 212628098.01 as a false positive due to the large implied planet radius of $14-18 R_{\oplus}$ and the presence of a neighboring star only 1 ". 25 from the target star. The nearby star is only 3.8 magnitudes fainter than the target star and was detected both in Gemini-N/NIRI AO images and in speckle images acquired with DSSI at Gemini-S and WIYN.

\subsection{Assessing False Positive Probabilities}

For the more promising K2OIs, we used the VESPA framework to assess the probabilities that each was truly a transiting planet. We first fit the transit photometry as described in Section 4 and rescaled the photometric errors so that the adopted transit model had a reduced chi squared of 1 for the segment of the light curve centered on the transit event. Prior to rescaling the errors, we clipped the light curves and kept only the points within six durations of the transit center. We then searched for secondary eclipses by phase-folding the full data set to the orbital period of the planet in question and measuring the "eclipse depth" at multiple points in the light curve. We assumed that the eclipse has the same duration as the primary transit, but we allowed the phase of secondary eclipse to vary from phase $=0.3$ to phase $=0.7$ (i.e., we did not assume that the orbit is circular). We recorded the depth of the deepest event as the maximum allowed secondary eclipse depth ("secthresh" in the VESPA fpp. ini file).

Next, we referred back to the K2SFF photometry and recorded the radius of the selected photometric aperture as the maximum allowed separation between the target and the source of the transit event ("maxrad"). We then consulted previously published K2 papers and the Exo-FOP K2 follow-up website to check whether there are extant speckle or high-contrast images

\footnotetext{
${ }^{24}$ https://exofop.ipac.caltech.edu/k2/
}

placing limits on the allowed brightness of nearby stars. If so, we included those contrast curves as additional constraints. For reference, we list the adaptive optics observations used in our false positive analysis in Table 1 and display the composite set of contrast curves in Figure 1. These observations were obtained with NIRC2 on the $10 \mathrm{~m}$ Keck II telescope, NIRI (Hodapp et al. 2003) on the $8 \mathrm{~m}$ Gemini-N telescope, PHARO (Hayward et al. 2001) on the 200 inch Palomar Hale telescope, and DSSI (Howell et al. 2011; Horch et al. 2012) on the $8 \mathrm{~m}$ Gemini-N and Gemini-S telescopes and the $3.5 \mathrm{~m} \mathrm{WIYN}^{25}$ telescope.

Finally, we filled in the host star properties (coordinates, magnitudes, and spectroscopic fits from D17) and ran VESPA to compute the false positive probability (FPP). Recognizing that VESPA FPPs are statistical and depend on the assumed planet radius, we ran the analysis twice for each planet using $R_{p} / R_{\star}$ ratios set to the 16 th and 84 th percentiles of the posterior distribution. As in Crossfield et al. (2016), we adopted a threshold of FPP $<1 \%$ for validation, but we adopted a less forgiving FPP cut of FPP $>90 \%$. When classifying K2OIs, we required that both FPP estimates were below $1 \%$ or above $90 \%$ in order to label K2OIs as validated planets or false positives, respectively. We classified the remaining systems as planet candidates.

We summarize our new K2OI dispositions in Table 2. In total, 44 K2OIs met the formal criteria for validation, but seven orbit stars with nearby companions and therefore cannot be validated with VESPA. Of the remaining 37 K2OIs with low FPPs, 20 were previously validated by Crossfield et al. (2016), eight are new detections, and nine were previously classified as planet candidates. As discussed in Section 5.2, we rejected eight K2OIs as false positives based on visual inspection of their light curves. No additional K2OIs were classified as false positives based on VESPA FPPs alone. The remaining 27 K2OIs had ambiguous FPPs between $1 \%$ and $90 \%$. The ambiguous sample included three previously confirmed planets, nine previously announced planet candidates, and 15 new detections. The three confirmed planets that failed to meet our 1\% FPP threshold are EPIC 201345483.01 $\left(R_{p}=10.4 R_{\oplus}\right.$, FPP $\left.=15 \%\right)$, EPIC 20163 $5569.01\left(R_{p}=7.5 R_{\oplus}, \mathrm{FPP}=4 \%-7 \%\right)$, and EPIC 210508766.02 $\left(R_{p}=2.1 R_{\oplus}, \mathrm{FPP}=1.9 \%\right)$. One other previously confirmed planet (EPIC 201637175.01 = K2-22b, Sanchis-Ojeda et al. 2015) met our FPP cut for validation, but is listed as a planet candidate in Table 2 due to the presence of a nearby star. We do not dispute the previous validation of $\mathrm{K} 2-22 \mathrm{~b}$, but our VESPA analysis is not sufficiently sophisticated to validate planets orbiting stars with nearby companions.

The most likely explanation for why we were unable to validate EPIC 201345483.01 is that our estimates of the stellar radius $\left(0.69_{-0.04}^{+0.06} R_{\odot}, \mathrm{D} 17\right)$ and planet radius $\left(10.4_{-0.7}^{+0.9} R_{\oplus}\right)$ are much larger than the values of $0.445 \pm 0.066 R_{\odot}$ and $6.71 R_{\oplus}$ assumed by Crossfield et al. (2016) when validating the system. In contrast, our estimates agree well with those adopted by Vanderburg et al. (2016b) in their discovery paper $\left(0.66 R_{\odot}\right.$, $\left.11 R_{\oplus}\right)$. Because larger planets are rarer than smaller planets, our larger planet radius estimate led us to adopt a smaller prior probability of planethood than Crossfield et al. (2016) based on their smaller planet radius estimate. In the future, including AO or speckle imaging would be useful for discriminating between

\footnotetext{
25 The WIYN Observatory is a joint facility of the University of WisconsinMadison, Indiana University, the National Optical Astronomy Observatory and the University of Missouri and hosts the NASA-NSF NN-EXPLORE program.
} 
Table 1

Speckle and AO Observations Used in VESPA Analysis

\begin{tabular}{|c|c|c|c|c|c|c|c|c|c|c|c|}
\hline \multirow[b]{2}{*}{ EPIC } & \multirow[b]{2}{*}{ Telescope } & \multirow[b]{2}{*}{ Instrument } & \multirow[b]{2}{*}{ Filter } & \multirow{2}{*}{$\begin{array}{l}\text { Pixel } \\
\text { Scale }\end{array}$} & \multirow{2}{*}{$\begin{array}{l}\text { PSF } \\
\left({ }^{\prime \prime}\right)\end{array}$} & \multicolumn{3}{|c|}{ Nearby Star $^{\mathrm{a}}$} & \multirow{2}{*}{$\begin{array}{l}\text { Contrast } \\
\text { Achieved }^{\mathrm{b}}\end{array}$} & \multirow{2}{*}{$\begin{array}{c}\text { Observation } \\
\text { Date }\end{array}$} & \multirow{2}{*}{$\begin{array}{l}\text { Uploaded } \\
\text { By }\end{array}$} \\
\hline & & & & & & $\overline{\text { Det? }}$ & $\Delta \mathrm{mag}$ & $\overline{\operatorname{Sep}\left({ }^{\prime \prime}\right)}$ & & & \\
\hline 201205469 & Keck2_10m & NIRC2 & $\mathrm{K}$ & 0.009942 & 0.066557 & no & $\ldots$ & $\ldots$ & $\Delta 7.91 \mathrm{mag}$ at 0.5 & $4 / 7 / 15$ & Ciardi \\
\hline 201208431 & Keck2_10m & NIRC2 & $\mathrm{K}$ & 0.009942 & 0.079626 & no & $\ldots$ & $\cdots$ & $\Delta 5.86 \mathrm{mag}$ at 0.5 & $2 / 19 / 16$ & Ciardi \\
\hline 201549860 & Keck2_10m & NIRC2 & $\mathrm{K}$ & 0.009942 & 0.060571 & no & $\cdots$ & $\cdots$ & $\Delta 8.10 \mathrm{mag}$ at $0 . " 5$ & $4 / 1 / 15$ & Ciardi \\
\hline 201617985 & Keck2_10m & NIRC2 & $\mathrm{K}$ & 0.009942 & 0.091839 & no & $\cdots$ & $\cdots$ & $\Delta 7.84 \mathrm{mag}$ at $0 . \prime 5$ & $4 / 1 / 15$ & Ciardi \\
\hline 201637175 & GeminiN_8m & DSSI & $692 \mathrm{~nm}$ & 0.011410 & 0.020000 & no ${ }^{c}$ & $\cdots$ & $\cdots$ & $\Delta 4.977 \mathrm{mag}$ at $0 . " 5$ & $1 / 15 / 16$ & Ciardi \\
\hline 201637175 & GeminiN_8m & DSSI & $880 \mathrm{~nm}$ & 0.011410 & 0.020000 & $\mathrm{no}^{\mathrm{c}}$ & $\cdots$ & $\cdots$ & $\Delta 4.666 \mathrm{mag}$ at 0.5 & $1 / 15 / 16$ & Ciardi \\
\hline 201855371 & Keck2_10m & NIRC2 & $\mathrm{K}$ & 0.009942 & 0.053694 & no & $\cdots$ & $\cdots$ & $\Delta 8.49 \mathrm{mag}$ at $0 . " 5$ & $4 / 7 / 15$ & Ciardi \\
\hline 205924614 & Keck2_10m & NIRC2 & $\mathrm{K}$ & 0.009942 & 0.058868 & no & $\cdots$ & $\cdots$ & $\Delta 8.17 \mathrm{mag}$ at $0 . " 5$ & $8 / 7 / 15$ & Ciardi \\
\hline 206011691 & Keck2_10m & NIRC2 & $\mathrm{K}$ & 0.009942 & 0.050896 & no & $\cdots$ & $\cdots$ & $\Delta 7.45 \mathrm{mag}$ at $0 . " 5$ & $7 / 25 / 15$ & Ciardi \\
\hline 206209135 & Keck2_10m & NIRC2 & $\mathrm{K}$ & 0.009942 & 0.062750 & no & $\cdots$ & $\cdots$ & $\Delta 7.74 \mathrm{mag}$ at 0.15 & $8 / 21 / 15$ & Ciardi \\
\hline 210448987 & GeminiN_8m & NIRI & $\mathrm{K}$ & 0.021400 & 0.102616 & no & $\cdots$ & $\cdots$ & $\Delta 6.86 \mathrm{mag}$ at $0 . " 5$ & $12 / 14 / 15$ & Ciardi \\
\hline 210489231 & Keck2_10m & NIRC2 & $\mathrm{K}$ & 0.009942 & 0.101436 & no & $\cdots$ & $\cdots$ & $\Delta 5.78 \mathrm{mag}$ at $0 . " 5$ & $10 / 28 / 15$ & Ciardi \\
\hline 210508766 & Keck2_10m & NIRC2 & $\mathrm{K}$ & 0.009942 & 0.054223 & no & $\cdots$ & $\cdots$ & $\Delta 7.40 \mathrm{mag}$ at $0 . " 5$ & $10 / 28 / 15$ & Ciardi \\
\hline 210508766 & GeminiN_8m & DSSI & $692 \mathrm{~nm}$ & 0.011410 & 0.020000 & no & $\cdots$ & $\cdots$ & $\Delta 4.994 \mathrm{mag}$ at $0 . " 5$ & $1 / 13 / 16$ & Ciardi \\
\hline 210508766 & GeminiN_8m & DSSI & $880 \mathrm{~nm}$ & 0.011410 & 0.020000 & no & $\cdots$ & $\cdots$ & $\Delta 5.248 \mathrm{mag}$ at 0.5 & $1 / 13 / 16$ & Ciardi \\
\hline 210508766 & Palomar-5 m & PHARO-AO & K_short & 0.025000 & 0.159000 & no & $\cdots$ & $\cdots$ & $\Delta 4.90 \mathrm{mag}$ at 0.5 & $10 / 20 / 16$ & Ciardi \\
\hline 210558622 & Keck2_10m & NIRC2 & $\mathrm{K}$ & 0.009942 & 0.049441 & $\mathrm{no}^{\mathrm{d}}$ & $\cdots$ & $\ldots$ & $\Delta 7.86 \mathrm{mag}$ at 0.5 & $10 / 28 / 15$ & Ciardi \\
\hline 210558622 & Keck2_10m & NIRC2 & $\mathrm{K}$ & 0.009942 & 0.050778 & no ${ }^{\mathrm{d}}$ & $\cdots$ & $\cdots$ & $\Delta 8.24 \mathrm{mag}$ at 0.5 & $2 / 17 / 16$ & Ciardi \\
\hline 210558622 & GeminiN_8m & DSSI & $692 \mathrm{~nm}$ & 0.011410 & 0.020000 & no $^{\mathrm{d}}$ & $\cdots$ & $\cdots$ & $\Delta 5.286 \mathrm{mag}$ at $0 . " 5$ & $1 / 13 / 16$ & Ciardi \\
\hline 210558622 & GeminiN_8m & DSSI & $880 \mathrm{~nm}$ & 0.011410 & 0.020000 & no ${ }^{\mathrm{d}}$ & $\cdots$ & $\cdots$ & $\Delta 5.248 \mathrm{mag}$ at $0 . " 5$ & $1 / 13 / 16$ & Ciardi \\
\hline 210558622 & WIYN_3.5 m & DSSI & $692 \mathrm{~nm}$ & 0.022000 & 0.050000 & no ${ }^{\mathrm{d}}$ & $\ldots$ & $\cdots$ & $\Delta 3.5 \mathrm{mag}$ at $0 .{ }^{\prime \prime} 2$ & $10 / 24 / 15$ & Everett \\
\hline 210558622 & WIYN_3.5 m & DSSI & $880 \mathrm{~nm}$ & 0.022000 & 0.063000 & $\mathrm{no}^{\mathrm{d}}$ & $\cdots$ & $\cdots$ & $\Delta 3.5 \mathrm{mag}$ at $0 . \prime 2$ & $10 / 24 / 15$ & Everett \\
\hline 210707130 & Keck2_10m & NIRC2 & $\mathrm{K}$ & 0.009942 & 0.051244 & no & $\cdots$ & $\cdots$ & $\Delta 7.92 \mathrm{mag}$ at 0.15 & $10 / 28 / 15$ & Ciardi \\
\hline 210707130 & Keck2_10m & NIRC2 & $\mathrm{K}$ & 0.009942 & 0.053366 & no & $\cdots$ & $\cdots$ & $\Delta 6.62 \mathrm{mag}$ at $0 . " 5$ & $2 / 19 / 16$ & Ciardi \\
\hline 210707130 & GeminiN_8m & DSSI & $692 \mathrm{~nm}$ & 0.011410 & 0.020000 & no & $\ldots$ & $\cdots$ & $\Delta 5.650 \mathrm{mag}$ at 0.5 & $1 / 13 / 16$ & Ciardi \\
\hline 210707130 & GeminiN_8m & DSSI & $880 \mathrm{~nm}$ & 0.011410 & 0.020000 & no & $\cdots$ & $\cdots$ & $\Delta 5.719 \mathrm{mag}$ at $0 . " 5$ & $1 / 13 / 16$ & Ciardi \\
\hline 210750726 & Keck2_10m & NIRC2 & $\mathrm{K}$ & 0.009942 & 0.111601 & no & $\cdots$ & $\cdots$ & $\Delta 5.36 \mathrm{mag}$ at $0 . " 5$ & $10 / 28 / 15$ & Ciardi \\
\hline 210750726 & Keck2_10m & NIRC2 & $\mathrm{K}$ & 0.009942 & 0.080981 & no & $\cdots$ & $\cdots$ & $\Delta 6.06 \mathrm{mag}$ at $0 . " 5$ & $2 / 19 / 16$ & Ciardi \\
\hline 210750726 & GeminiN_8m & DSSI & $692 \mathrm{~nm}$ & 0.011410 & 0.020000 & no & $\cdots$ & $\cdots$ & $\Delta 4.901 \mathrm{mag}$ at $0 . \prime 5$ & $1 / 15 / 16$ & Ciardi \\
\hline 210750726 & GeminiN_8m & DSSI & $880 \mathrm{~nm}$ & 0.011410 & 0.020000 & no & $\cdots$ & $\cdots$ & $\Delta 5.002 \mathrm{mag}$ at $0 . " 5$ & $1 / 15 / 16$ & Ciardi \\
\hline 210838726 & GeminiN_8m & NIRI & $\mathrm{K}$ & 0.021400 & 0.109663 & no & $\cdots$ & $\cdots$ & $\Delta 6.12 \mathrm{mag}$ at $0 . " 5$ & $11 / 2 / 15$ & Ciardi \\
\hline 210838726 & GeminiN_8m & NIRI & $\mathrm{K}$ & 0.021400 & 0.099683 & no & $\cdots$ & $\cdots$ & $\Delta 6.83 \mathrm{mag}$ at $0 . " 5$ & $12 / 14 / 15$ & Ciardi \\
\hline 210968143 & Keck2_10m & NIRC2 & $\mathrm{K}$ & 0.009942 & 0.053846 & no & $\cdots$ & $\cdots$ & $\Delta 7.59 \mathrm{mag}$ at $0 . " 5$ & $10 / 28 / 15$ & Ciardi \\
\hline 210968143 & Keck2_10m & NIRC2 & $\mathrm{K}$ & 0.009942 & 0.051648 & no & $\ldots$ & $\cdots$ & $\Delta 7.91 \mathrm{mag}$ at $0 . " 5$ & $2 / 17 / 16$ & Ciardi \\
\hline 210968143 & GeminiN_8m & DSSI & $692 \mathrm{~nm}$ & 0.011410 & 0.020000 & no & $\cdots$ & $\cdots$ & $\Delta 5.279 \mathrm{mag}$ at $0 . " 5$ & $1 / 13 / 16$ & Ciardi \\
\hline 210968143 & GeminiN_8m & DSSI & $880 \mathrm{~nm}$ & 0.011410 & 0.020000 & no & $\cdots$ & $\cdots$ & $\Delta 5.089 \mathrm{mag}$ at $0 . \prime 5$ & $1 / 13 / 16$ & Ciardi \\
\hline 210968143 & Palomar-5 m & PHARO-AO & K_short & 0.025000 & 0.165000 & no & $\cdots$ & $\cdots$ & $\Delta 4.73 \mathrm{mag}$ at $0 . \prime 5$ & $10 / 20 / 16$ & Ciardi \\
\hline 211077024 & Keck2_10m & NIRC2 & K & 0.009942 & 0.061843 & no & $\cdots$ & $\cdots$ & $\Delta 7.41 \mathrm{mag}$ at 0.5 & $2 / 19 / 16$ & Ciardi \\
\hline 211077024 & GeminiN_8m & DSSI & $692 \mathrm{~nm}$ & 0.011410 & 0.020000 & no & $\cdots$ & $\cdots$ & $\Delta 4.924 \mathrm{mag}$ at $0 . " 5$ & $1 / 15 / 16$ & Ciardi \\
\hline 211077024 & GeminiN_8m & DSSI & $880 \mathrm{~nm}$ & 0.011410 & 0.020000 & no & $\cdots$ & $\cdots$ & $\Delta 5.371 \mathrm{mag}$ at $0 . " 5$ & $1 / 15 / 16$ & Ciardi \\
\hline 211331236 & Keck2_10m & NIRC2 & $\mathrm{K}$ & 0.009942 & 0.079162 & no & $\cdots$ & $\cdots$ & $\Delta 6.62 \mathrm{mag}$ at $0 . \prime 5$ & $1 / 21 / 16$ & Ciardi \\
\hline 211428897 & Keck2_10m & NIRC2 & $\mathrm{J}$ & 0.009942 & 0.114165 & yes & $\cdots$ & $\cdots$ & $\Delta 5.41 \mathrm{mag}$ at $0 . " 5$ & $1 / 21 / 16$ & Ciardi \\
\hline 211428897 & Keck2_10m & NIRC2 & $\mathrm{K}$ & 0.009942 & 0.060653 & yes & $\cdots$ & $\cdots$ & $\Delta 6.86 \mathrm{mag}$ at 0.5 & $1 / 21 / 16$ & Ciardi \\
\hline 211428897 & GeminiN_8m & DSSI & $692 \mathrm{~nm}$ & 0.011410 & 0.020000 & yes & 1.8 & 1.1 & $\Delta 4.442 \mathrm{mag}$ at $0 . " 5$ & $1 / 15 / 16$ & Ciardi \\
\hline 211428897 & GeminiN_8m & DSSI & $880 \mathrm{~nm}$ & 0.011410 & 0.020000 & yes & 1.2 & 1.1 & $\Delta 4.869 \mathrm{mag}$ at $0 . " 5$ & $1 / 15 / 16$ & Ciardi \\
\hline
\end{tabular}


Table 1

(Continued)

\begin{tabular}{|c|c|c|c|c|c|c|c|c|c|c|c|}
\hline \multirow[b]{2}{*}{ EPIC } & \multirow[b]{2}{*}{ Telescope } & \multirow[b]{2}{*}{ Instrument } & \multirow[b]{2}{*}{ Filter } & \multirow{2}{*}{$\begin{array}{l}\text { Pixel } \\
\text { Scale }\end{array}$} & \multirow{2}{*}{$\begin{array}{c}\text { PSF } \\
\left({ }^{\prime \prime}\right)\end{array}$} & \multicolumn{3}{|c|}{ Nearby $\operatorname{Star}^{\mathrm{a}}$} & \multirow{2}{*}{$\begin{array}{l}\text { Contrast } \\
\text { Achieved }^{\mathrm{b}}\end{array}$} & \multirow{2}{*}{$\begin{array}{c}\text { Observation } \\
\text { Date }\end{array}$} & \multirow{2}{*}{$\begin{array}{l}\text { Uploaded } \\
\text { By }\end{array}$} \\
\hline & & & & & & Det? & $\Delta \mathrm{mag}$ & Sep $\left({ }^{\prime \prime}\right)$ & & & \\
\hline 211509553 & GeminiN-8 m & NIRI & open & 0.021400 & 0.098000 & yes & 3.3 & 1.9 & $\Delta 5.84 \mathrm{mag}$ at $0 . " 5$ & $2 / 20 / 16$ & Ciardi \\
\hline 211770795 & Keck2_10m & NIRC2 & $\mathrm{K}$ & 0.009942 & 0.065018 & no & $\ldots$ & $\ldots$ & $\Delta 7.36 \mathrm{mag}$ at $0 . " 5$ & $2 / 19 / 16$ & Ciardi \\
\hline 211770795 & GeminiN-8 m & NIRI & open & 0.021400 & 0.112000 & no & $\cdots$ & $\cdots$ & $\Delta 5.57 \mathrm{mag}$ at $0 . " 5$ & $2 / 20 / 16$ & Ciardi \\
\hline 211799258 & GeminiN-8 m & NIRI & open & 0.021400 & 0.107000 & no & $\cdots$ & $\cdots$ & $\Delta 6.61 \mathrm{mag}$ at $0 . " 5$ & $2 / 20 / 16$ & Ciardi \\
\hline 211818569 & Palomar-5 m & PHARO-AO & Ks & 0.025000 & 0.122000 & no & $\cdots$ & $\cdots$ & $\Delta 5.22 \mathrm{mag}$ at $0 . " 5$ & $10 / 20 / 16$ & Ciardi \\
\hline 211831378 & GeminiN_8m & NIRI & $\mathrm{K}$ & 0.021400 & 0.125410 & no & $\cdots$ & $\cdots$ & $\Delta 6.59 \mathrm{mag}$ at $0 . " 5$ & $1 / 28 / 16$ & Ciardi \\
\hline 211924657 & GeminiN-8 m & NIRI & Br-gamma & 0.021400 & 0.101000 & no & $\cdots$ & $\cdots$ & $\Delta 6.26 \mathrm{mag}$ at $0 . " 5$ & $2 / 20 / 16$ & Ciardi \\
\hline 211970234 & GeminiN-8 m & NIRI & open & 0.021400 & 0.113000 & no & $\cdots$ & $\cdots$ & $\Delta 5.60 \mathrm{mag}$ at $0 . " 5$ & $2 / 20 / 16$ & Ciardi \\
\hline 212006344 & Keck2_10m & NIRC2 & $\mathrm{K}$ & 0.009942 & 0.053540 & no & $\cdots$ & $\cdots$ & $\Delta 8.06 \mathrm{mag}$ at $0 . " 5$ & $1 / 21 / 16$ & Ciardi \\
\hline 212006344 & GeminiN_8m & DSSI & $692 \mathrm{~nm}$ & 0.011410 & 0.020000 & no & $\cdots$ & $\cdots$ & $\Delta 5.281 \mathrm{mag}$ at $0 . .5$ & $1 / 14 / 16$ & Ciardi \\
\hline 212006344 & GeminiN_8m & DSSI & $880 \mathrm{~nm}$ & 0.011410 & 0.020000 & no & $\cdots$ & $\cdots$ & $\Delta 5.800 \mathrm{mag}$ at $0 . " 5$ & $1 / 14 / 16$ & Ciardi \\
\hline 212069861 & Keck2_10m & NIRC2 & $\mathrm{J}$ & 0.009942 & 0.092989 & no & $\cdots$ & $\cdots$ & $\Delta 6.17 \mathrm{mag}$ at $0 . " 5$ & $2 / 17 / 16$ & Ciardi \\
\hline 212069861 & Keck2_10m & NIRC2 & K & 0.009942 & 0.096097 & no & $\cdots$ & $\cdots$ & $\Delta 7.63 \mathrm{mag}$ at $0 . " 5$ & $2 / 17 / 16$ & Ciardi \\
\hline 212069861 & GeminiN-8 m & NIRI & Br-gamma & 0.021400 & 0.131000 & no & $\cdots$ & $\cdots$ & $\Delta 4.93 \mathrm{mag}$ at $0 . " 5$ & $2 / 20 / 16$ & Ciardi \\
\hline 212154564 & Keck2_10m & NIRC2 & $\mathrm{K}$ & 0.009942 & 0.111359 & no & $\cdots$ & $\cdots$ & $\Delta 6.06 \mathrm{mag}$ at $0 . " 5$ & $2 / 19 / 16$ & Ciardi \\
\hline 212154564 & GeminiN-8 m & NIRI & open & 0.021400 & 0.106000 & no & $\cdots$ & $\cdots$ & $\Delta 5.52 \mathrm{mag}$ at $0 . " 5$ & $2 / 20 / 16$ & Ciardi \\
\hline 212354731 & GeminiS_8m & DSSI & $692 \mathrm{~nm}$ & 0.011000 & 0.021000 & no & $\cdots$ & $\cdots$ & $\Delta 5 \mathrm{mag}$ at $0 . \prime 2$ & $6 / 29 / 16$ & Everett \\
\hline 212354731 & GeminiS_8m & DSSI & $880 \mathrm{~nm}$ & 0.011000 & 0.027000 & no & $\cdots$ & $\cdots$ & $\Delta 5 \mathrm{mag}$ at $0 ! 2$ & $6 / 29 / 16$ & Everett \\
\hline 212460519 & WIYN_3.5 m & DSSI & $692 \mathrm{~nm}$ & 0.022000 & 0.050000 & no & $\cdots$ & $\cdots$ & $\Delta 3.5 \mathrm{mag}$ at $0 . \prime 2$ & $4 / 21 / 16$ & Everett \\
\hline 212460519 & WIYN_3.5 m & DSSI & $880 \mathrm{~nm}$ & 0.022000 & 0.063000 & no & $\cdots$ & $\cdots$ & $\Delta 3.5 \mathrm{mag}$ at 0.2 & $4 / 21 / 16$ & Everett \\
\hline 212554013 & GeminiS_8m & DSSI & $692 \mathrm{~nm}$ & 0.011000 & 0.021000 & no & $\cdots$ & $\cdots$ & $\Delta 5 \mathrm{mag}$ at $0 . ! 2$ & $6 / 22 / 16$ & Everett \\
\hline 212554013 & GeminiS_8m & DSSI & $880 \mathrm{~nm}$ & 0.011000 & 0.027000 & no & $\ldots$ & $\cdots$ & $\Delta 5 \mathrm{mag}$ at $0 . ! 2$ & $6 / 22 / 16$ & Everett \\
\hline 212565386 & GeminiS_8m & DSSI & $692 \mathrm{~nm}$ & 0.011000 & 0.021000 & no & $\cdots$ & $\cdots$ & $\Delta 5 \mathrm{mag}$ at $0 . ! 2$ & $6 / 22 / 16$ & Everett \\
\hline 212565386 & GeminiS_8m & DSSI & $880 \mathrm{~nm}$ & 0.011000 & 0.027000 & no & $\cdots$ & $\cdots$ & $\Delta 5 \mathrm{mag}$ at $0 . ! 2$ & $6 / 22 / 16$ & Everett \\
\hline 212572452 & GeminiS_8m & DSSI & $692 \mathrm{~nm}$ & 0.011000 & 0.021000 & no & $\cdots$ & $\cdots$ & $\Delta 5 \mathrm{mag}$ at $0 . \prime 2$ & $6 / 22 / 16$ & Everett \\
\hline 212572452 & GeminiS_8m & DSSI & $880 \mathrm{~nm}$ & 0.011000 & 0.027000 & no & $\cdots$ & $\cdots$ & $\Delta 5 \mathrm{mag}$ at $0 ! ! 2$ & $6 / 22 / 16$ & Everett \\
\hline 212628098 & GeminiS_8m & DSSI & $692 \mathrm{~nm}$ & 0.011000 & 0.021000 & yes & $\cdots$ & $\cdots$ & $\Delta 5 \mathrm{mag}$ at $0 ! 2$ & $6 / 22 / 16$ & Everett \\
\hline 212628098 & GeminiS_8m & DSSI & $880 \mathrm{~nm}$ & 0.011000 & 0.027000 & yes & 3.8 & 1.3 & $\Delta 5 \mathrm{mag}$ at $0 . " 2$ & $6 / 22 / 16$ & Everett \\
\hline 212628098 & WIYN_3.5 m & DSSI & $692 \mathrm{~nm}$ & 0.022000 & 0.050000 & yes & $\cdots$ & $\cdots$ & $\Delta 3.5 \mathrm{mag}$ at $0 . \prime 2$ & $4 / 20 / 16$ & Everett \\
\hline 212628098 & WIYN_3.5 m & DSSI & $880 \mathrm{~nm}$ & 0.022000 & 0.063000 & yes & $\cdots$ & $\cdots$ & $\Delta 3.5 \mathrm{mag}$ at $0 . " 2$ & $4 / 20 / 16$ & Everett \\
\hline 212628098 & GeminiN-8 m & NIRI & Br-gamma & 0.021400 & 0.107000 & yes & $\cdots$ & $\cdots$ & $\Delta 7.01 \mathrm{mag}$ at 0.5 & $6 / 20 / 16$ & Ciardi \\
\hline 212679181 & GeminiS_8m & DSSI & $692 \mathrm{~nm}$ & 0.011000 & 0.021000 & yes & 1.1 & 1.2 & $\Delta 5 \mathrm{mag}$ at $0 . \prime 2$ & $6 / 21 / 16$ & Everett \\
\hline 212679181 & GeminiS_8m & DSSI & $880 \mathrm{~nm}$ & 0.011000 & 0.027000 & yes & 1.1 & 1.3 & $\Delta 5 \mathrm{mag}$ at $0 .{ }^{\prime \prime} 2$ & $6 / 21 / 16$ & Everett \\
\hline 212679181 & WIYN_3.5 m & DSSI & $692 \mathrm{~nm}$ & 0.022000 & 0.050000 & yes & 1.5 & 1.5 & $\Delta 3.5 \mathrm{mag}$ at 0.2 & $4 / 17 / 16$ & Everett \\
\hline 212679181 & WIYN_3.5 m & DSSI & $880 \mathrm{~nm}$ & 0.022000 & 0.063000 & yes & 1.2 & 1.5 & $\Delta 3.5 \mathrm{mag}$ at $0 . " 2$ & $4 / 17 / 16$ & Everett \\
\hline 212679798 & GeminiS_8m & DSSI & $692 \mathrm{~nm}$ & 0.011000 & 0.021000 & yes & $\cdots$ & $\cdots$ & $\Delta 5 \mathrm{mag}$ at $0 . \prime 2$ & $6 / 22 / 16$ & Everett \\
\hline 212679798 & GeminiS_8m & DSSI & $880 \mathrm{~nm}$ & 0.011000 & 0.027000 & yes & 2.6 & 0.1 & $\Delta 5 \mathrm{mag}$ at $0 . \prime 2$ & $6 / 22 / 16$ & Everett \\
\hline 212679798 & GeminiN-8 m & NIRI & open & 0.021400 & 0.110000 & yes & $\cdots$ & $\cdots$ & $\Delta 6.62 \mathrm{mag}$ at $0 . " 5$ & $6 / 20 / 16$ & Ciardi \\
\hline 212686205 & WIYN_3.5 m & DSSI & $692 \mathrm{~nm}$ & 0.022000 & 0.050000 & no & $\cdots$ & $\ldots$ & $\Delta 3.5 \mathrm{mag}$ at 0.2 & $4 / 20 / 16$ & Everett \\
\hline 212686205 & WIYN_3.5 m & DSSI & $880 \mathrm{~nm}$ & 0.022000 & 0.063000 & no & $\cdots$ & $\cdots$ & $\Delta 3.5 \mathrm{mag}$ at $0 . \prime 2$ & $4 / 20 / 16$ & Everett \\
\hline 212773272 & GeminiS_8m & DSSI & $692 \mathrm{~nm}$ & 0.011000 & 0.021000 & no & $\cdots$ & $\cdots$ & $\Delta 5 \mathrm{mag}$ at 0.2 & $6 / 21 / 16$ & Everett \\
\hline 212773272 & GeminiS_8m & DSSI & $880 \mathrm{~nm}$ & 0.011000 & 0.027000 & no & $\cdots$ & $\cdots$ & $\Delta 5 \mathrm{mag}$ at $0 . ! 2$ & $6 / 21 / 16$ & Everett \\
\hline 212773272 & GeminiN-8 m & NIRI & Br-gamma & 0.021400 & 0.109000 & no & $\cdots$ & $\cdots$ & $\Delta 7.09 \mathrm{mag}$ at $0 . " 5$ & $6 / 20 / 16$ & Ciardi \\
\hline 212773309 & GeminiS_8m & DSSI & $692 \mathrm{~nm}$ & 0.011000 & 0.021000 & yes & 2.8 & 1.0 & $\Delta 5 \mathrm{mag}$ at $0 . " 2$ & $6 / 21 / 16$ & Everett \\
\hline
\end{tabular}


Table 1

(Continued)

\begin{tabular}{|c|c|c|c|c|c|c|c|c|c|c|c|}
\hline \multirow[b]{2}{*}{ EPIC } & \multirow[b]{2}{*}{ Telescope } & \multirow[b]{2}{*}{ Instrument } & \multirow[b]{2}{*}{ Filter } & \multirow{2}{*}{$\begin{array}{l}\text { Pixel } \\
\text { Scale }\end{array}$} & \multirow{2}{*}{$\begin{array}{l}\text { PSF } \\
\left({ }^{\prime \prime}\right)\end{array}$} & \multicolumn{3}{|c|}{ Nearby $S a^{a}$} & \multirow{2}{*}{$\begin{array}{l}\text { Contrast } \\
\text { Achieved }^{\mathrm{b}}\end{array}$} & \multirow{2}{*}{$\begin{array}{l}\text { Observation } \\
\text { Date }\end{array}$} & \multirow{2}{*}{$\begin{array}{l}\text { Uploaded } \\
\text { By }\end{array}$} \\
\hline & & & & & & $\overline{\text { Det? }}$ & $\Delta \mathrm{mag}$ & Sep $\left(^{(\prime)}\right.$ & & & \\
\hline 212773309 & GeminiS_8m & DSSI & $880 \mathrm{~nm}$ & 0.011000 & 0.027000 & yes & 2.0 & 1.0 & $\Delta 5 \mathrm{mag}$ at $0 . \prime 2$ & $6 / 21 / 16$ & Everett \\
\hline 212773309 & WIYN_3.5 m & DSSI & $692 \mathrm{~nm}$ & 0.022000 & 0.050000 & yes & 2.8 & 1.2 & $\Delta 3.5 \mathrm{mag}$ at $0 . ! 2$ & $4 / 24 / 16$ & Everett \\
\hline 212773309 & WIYN_3.5 m & DSSI & $880 \mathrm{~nm}$ & 0.022000 & 0.063000 & no & 2.0 & 1.2 & $\Delta 3.5 \mathrm{mag}$ at $0 .{ }^{\prime \prime} 2$ & $4 / 24 / 16$ & Everett \\
\hline 213951550 & GeminiN-8 m & NIRI & open & 0.021400 & 0.116000 & yes & $\cdots$ & 0.2 & $\Delta 6.41 \mathrm{mag}$ at $0 . " 5$ & $7 / 15 / 16$ & Ciardi \\
\hline 216892056 & GeminiS_8m & DSSI & $692 \mathrm{~nm}$ & 0.011000 & 0.021000 & no & $\cdots$ & $\cdots$ & $\Delta 5 \mathrm{mag}$ at $0 . ! 2$ & $6 / 29 / 16$ & Everett \\
\hline 216892056 & GeminiS_8m & DSSI & $880 \mathrm{~nm}$ & 0.011000 & 0.027000 & no & $\cdots$ & $\cdots$ & $\Delta 5 \mathrm{mag}$ at $0 . \prime 2$ & $6 / 29 / 16$ & Everett \\
\hline 216892056 & GeminiN-8 m & NIRI & Br-gamma & 0.021400 & 0.110000 & no & $\ldots$ & $\ldots$ & $\Delta 6.86 \mathrm{mag}$ at 0.15 & $6 / 15 / 16$ & Ciardi \\
\hline 217941732 & GeminiS_8m & DSSI & $692 \mathrm{~nm}$ & 0.011000 & 0.021000 & no & $\ldots$ & $\ldots$ & $\Delta 5 \mathrm{mag}$ at $0 . " 2$ & $6 / 19 / 16$ & Everett \\
\hline 217941732 & GeminiS_8m & DSSI & $880 \mathrm{~nm}$ & 0.011000 & 0.027000 & no & $\ldots$ & $\ldots$ & $\Delta 5 \mathrm{mag}$ at $0 . " 2$ & $6 / 19 / 16$ & Everett \\
\hline
\end{tabular}

Notes.

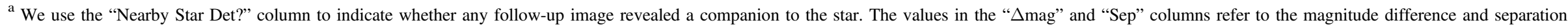
measured in the specific image described on the corresponding line of the table.

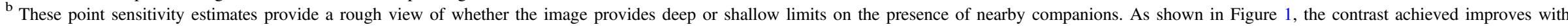
increasing separation from the target star. We use the full separation-dependent contrast curves for our false positive probability estimates.

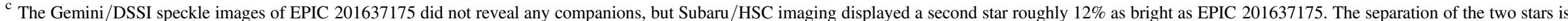
approximately $2^{\prime \prime}$ (Sanchis-Ojeda et al. 2015).

${ }^{\mathrm{d}}$ The follow-up images of EPIC 210558622 did not reveal any companions, but a second set of spectral lines was detected in the Keck/HIRES reconnaissance spectrum (Crossfield et al. 2016). 


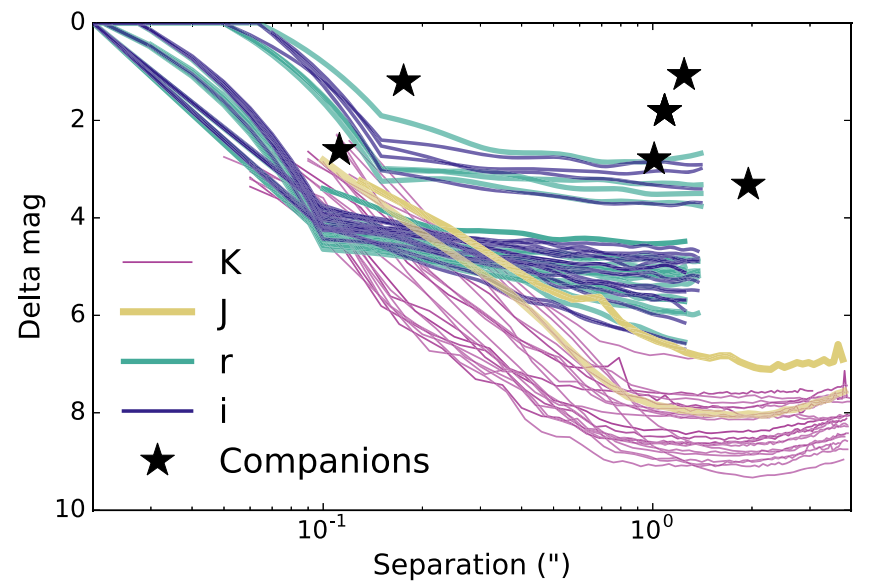

Figure 1. Sensitivity of our follow-up imaging observations as a function of separation. The purple and yellow contrast curves mark the limits achieved for our adaptive optics observations at near-infrared wavelengths, while the teal and navy curves display the limits for our speckle imaging at optical wavelengths. The stars indicate the magnitude difference between the K2 target stars and detected nearby "companion" stars that may or may not be physically associated. The magnitude differences shown here are those in the band used for the follow-up observations; the flux ratio in the $\mathrm{K} 2$ bandpass may be different.

Table 2

Breakdown of K2OI Dispositions

\begin{tabular}{lcccc}
\hline \hline \multirow{2}{*}{$\begin{array}{l}\text { Previous } \\
\text { Disposition }\end{array}$} & \multicolumn{4}{c}{ Updated Disposition $^{\mathrm{a}}$} \\
\cline { 2 - 5 } & CP & PC & FP & All \\
\hline CP & 22 & $3^{\text {b }}$ & 0 & $\mathbf{2 5}$ \\
PC & 9 & 13 & $1^{\text {c }}$ & $\mathbf{2 3}$ \\
FP & 0 & 0 & 2 & $\mathbf{2}$ \\
UK & 7 & 17 & 5 & $\mathbf{2 9}$ \\
\hline All & $\mathbf{3 8}^{\text {d }}$ & $\mathbf{3 3}$ & $\mathbf{8}$ & $\mathbf{7 9}$ \\
\hline
\end{tabular}

Notes.

${ }^{\mathrm{a}} \mathrm{CP}=$ Confirmed Planet, $\mathrm{PC}=$ Planet Candidate, $\mathrm{FP}=$ False Positive, $\mathrm{UK}=$ Unknown.

${ }^{\mathrm{b}}$ The previously confirmed planets that we cannot validate with VESPA are EPIC 201345483.01 (FPP = 15\%), EPIC 201635569.01 $(\mathrm{FPP}=4 \%-7 \%)$, and EPIC 201637175.01 (=K2-22b, nearby star detected). See Section 5.2 for details.

${ }^{\mathrm{c}}$ The planet candidate rejected as a false positive is EPIC 212572452.01, which was announced by Pope et al. (2016) as a candidate with $R_{p} / R_{\star}=0.174$ and an orbital period of 2.6 days. As discussed in Section 5.2, the K2 photometry for this target is contaminated by light from the nearby, brighter star EPIC 212572439.

${ }^{\mathrm{d}}$ Bold values indicate summations.

the planetary interpretation and remaining false positive scenarios.

EPIC 201635569.01 was previously validated by Montet et al. (2015) as a $4.81 \pm 0.42 R_{\oplus}$ planet with FPP $=4.9 \times 10^{-3}$ and by Crossfield et al. (2016) as a $4.48 \pm 0.52 R_{\oplus}$ planet with FPP $=0.6 \%$. Our inability to confirm the validation of this planet may be due to our larger estimate of the planet radius, which is in turn caused by the larger stellar radius found by D17. We estimated a revised radius of $0.62 \pm 0.03 R_{\odot}$, which is significantly larger than the values of $0.45 \pm 0.01 R_{\odot}$ and $0.39 \pm 0.04 R_{\odot}$ assumed by Montet et al. (2015) and Crossfield et al. (2016), respectively. Our new FPP estimate of $4 \%-7 \%$ is still consistent with the planetary interpretation of the transit-like event, but indicates that additional observations such as $\mathrm{AO}$ imaging would be useful to rule out the remaining false positive scenarios.

For EPIC 210508766.02, our initial FPP estimate of $1.9 \%$ is only slightly above our validation threshold of $1 \%$ and does not consider the fact that transit-like events detected in candidate multiple-planet systems are more likely to be bona fide planets (Lissauer et al. 2012). Given that EPIC 210508766 also hosts 210508766.01, we can apply a multiplicity boost to reduce the FPP for 210508766.02 by a factor of 30 (Sinukoff et al. 2016; Vanderburg et al. 2016a). We therefore support the previous validation of EPIC 210508766.02 (K2-83c) by Crossfield et al. (2016) and classify that K2OI as a validated planet while categorizing all of the other K2OIs with FPP between $1 \%$ and $90 \%$ as planet candidates. The final disposition breakdown for our K2OI sample is 38 validated planets, eight false positives, and 33 planet candidates.

We list the estimated FPPs and resulting dispositions for individual K2OIs in Table 3. For conciseness, Table 3 also includes estimates of the orbital periods and mid-points of transit-like events. We present the remaining transit parameters and the corresponding physical parameters in Table 4. As part of our classification and transit fitting process, we produced an array of vetting plots for each candidate. We have uploaded all of these plots to the ExoFOP-K2 website and provide examples in the Appendix.

As indicated in Figure 1 and Table 3, six of the planet candidates are associated with $\mathrm{K} 2$ targets for which our followup imaging observations revealed nearby companions and Keck/HIRES observations revealed an additional set of stellar lines in the spectrum of EPIC 210558622. The imaged companions might be physically associated with the target star or are simply background stars that fall along the same line of sight. Regardless, the close proximity of additional stars dilutes the depths of the transit-like events in the K2 light curves and causes the planets to appear smaller than their true size. In general, the radii of planet candidates orbiting stars with stellar companions are underestimated by a few percent if they orbit the target stars and by a factor of three if they orbit the companion stars (Furlan et al. 2017). Assessing whether the companion stars are bound to the target stars and determining the source of the transit events will require additional scrutiny of the K2 photometry and follow-up imagery (see Furlan et al. 2017, and references therein). We will discuss these systems in more detail in E. J. González et al. (2017, in preparation), an upcoming catalog paper describing the results of our follow-up images of candidate K2 planet host stars of all spectral types.

\section{Revised Planet Properties}

After refitting the transit photometry and calculating false positive probabilities, we combined our new transit parameters with updated stellar characterizations from D17 to determine the physical properties of each K2OI. We display the revised planet properties in Figure 2. The panels include the full population of K2 planet candidate, validated planets, and false positives as well as planet candidates and confirmed or validated planets identified during the original Kepler mission. In general, the left panel demonstrates that the planet size and orbital period distribution of our $\mathrm{K} 2$ planet candidates and validated planets is similar to the distribution of short-period Kepler planet candidates. The majority of planets and planet 
Table 3

K2OI False Positive Probabilities and Dispositions

\begin{tabular}{|c|c|c|c|c|c|c|c|c|c|c|c|c|c|c|c|}
\hline \multirow[b]{2}{*}{ EPIC } & \multirow[b]{2}{*}{$\mathrm{K} 2 \mathrm{OI}$} & \multicolumn{2}{|c|}{ Disposition } & \multicolumn{2}{|c|}{ VESPA FPP ${ }^{\mathrm{a}}$} & \multirow{2}{*}{$\begin{array}{l}\text { Nearby } \\
\text { Star? }\end{array}$} & \multicolumn{3}{|c|}{$\bar{R} R_{p} / R_{\star}$} & \multicolumn{3}{|c|}{$P$ (days) $^{\mathrm{c}}$} & \multicolumn{3}{|c|}{ t0 (BKJD) ${ }^{\mathrm{c}}$} \\
\hline & & Old & New & Small $R_{p}$ & $\operatorname{Big} R_{p}$ & & K2SFF & k2phot & $\overline{\mathrm{K} 2 \mathrm{SC}}$ & Val & - Err & + Err & Val & - Err & $+\mathrm{En}$ \\
\hline 201205469 & 1 & $\mathrm{CP}$ & $\mathrm{CP}$ & $3.6 \mathrm{e}-08$ & $3.6 \mathrm{e}-08$ & no & 0.074 & 0.072 & $\ldots$ & 3.47134 & 0.00016 & 0.00016 & 1976.881 & 0.002 & 0.002 \\
\hline 201208431 & 1 & $\mathrm{CP}$ & $\mathrm{CP}$ & $3.7 \mathrm{e}-07$ & $2.0 \mathrm{e}-06$ & no & 0.034 & 0.035 & & 10.00342 & 0.00100 & 0.00102 & 1982.524 & 0.004 & 0.004 \\
\hline 201345483 & 1 & $\mathrm{CP}$ & $\mathrm{PC}$ & $1.5 \mathrm{e}-01$ & $1.5 \mathrm{e}-01$ & $\ldots$ & 0.140 & 0.136 & $\ldots$ & 1.72926 & 0.00001 & 0.00001 & 1976.526 & 0.000 & 0.000 \\
\hline 201549860 & 1 & $\mathrm{CP}$ & $\mathrm{CP}$ & $2.3 \mathrm{e}-05$ & $3.4 \mathrm{e}-05$ & no & 0.028 & 0.028 & $\ldots$ & 5.60835 & 0.00034 & 0.00034 & 1979.119 & 0.002 & 0.002 \\
\hline 201549860 & 2 & $\mathrm{CP}$ & $\mathrm{CP}$ & $1.5 \mathrm{e}-09$ & $6.9 \mathrm{e}-08$ & no & 0.019 & 0.019 & $\ldots$ & 2.39996 & 0.00020 & 0.00020 & 1977.584 & 0.004 & 0.004 \\
\hline 201617985 & 1 & $\mathrm{PC}$ & $\mathrm{PC}$ & $5.0 \mathrm{e}-02$ & $8.3 \mathrm{e}-02$ & no & 0.033 & 0.033 & & 7.28118 & 0.00057 & 0.00055 & 1979.641 & 0.004 & 0.004 \\
\hline 201635569 & 1 & $\mathrm{CP}$ & $\mathrm{PC}$ & $3.6 \mathrm{e}-02$ & $6.7 \mathrm{e}-02$ & $\ldots$ & 0.110 & 0.104 & $\ldots$ & 8.36879 & 0.00019 & 0.00019 & 1978.447 & 0.001 & 0.001 \\
\hline 201637175 & 1 & $\mathrm{CP}$ & $\mathrm{PC}$ & $2.4 \mathrm{e}-03$ & $9.1 \mathrm{e}-03$ & yes & 0.075 & 0.074 & $\ldots$ & 0.38108 & 0.00000 & 0.00000 & 2034.139 & 0.000 & 0.000 \\
\hline 201717274 & 1 & $\mathrm{PC}$ & $\mathrm{PC}$ & $1.1 \mathrm{e}-01$ & $9.3 \mathrm{e}-02$ & $\ldots$ & 0.038 & 0.039 & $\ldots$ & 3.52675 & 0.00030 & 0.00030 & 1976.915 & 0.004 & 0.004 \\
\hline 201855371 & 1 & $\mathrm{CP}$ & $\mathrm{CP}$ & $6.2 \mathrm{e}-06$ & $1.4 \mathrm{e}-05$ & no & 0.030 & 0.030 & $\ldots$ & 17.96903 & 0.00142 & 0.00138 & 1984.944 & 0.003 & 0.003 \\
\hline 205924614 & 1 & $\mathrm{CP}$ & $\mathrm{CP}$ & $6.0 \mathrm{e}-11$ & $7.9 \mathrm{e}-11$ & no & 0.056 & 0.055 & 0.056 & 2.84927 & 0.00003 & 0.00003 & 2150.423 & 0.000 & 0.000 \\
\hline 206011691 & 1 & $\mathrm{CP}$ & $\mathrm{CP}$ & $4.1 \mathrm{e}-07$ & $1.0 \mathrm{e}-08$ & no & 0.026 & 0.026 & 0.026 & 9.32504 & 0.00040 & 0.00038 & 2156.422 & 0.001 & 0.001 \\
\hline 206011691 & 2 & $\mathrm{CP}$ & $\mathrm{CP}$ & $2.5 \mathrm{e}-08$ & $8.0 \mathrm{e}-13$ & no & 0.035 & 0.033 & 0.034 & 15.50192 & 0.00093 & 0.00092 & 2155.471 & 0.002 & 0.002 \\
\hline $206119924^{\mathrm{d}}$ & 1 & PC & $\mathrm{CP}$ & $2.6 \mathrm{e}-03$ & $2.2 \mathrm{e}-03$ & $\ldots$ & 0.009 & 0.009 & 0.009 & 4.65541 & 0.00047 & 0.00049 & 2146.948 & 0.004 & 0.00 \\
\hline 206209135 & 1 & $\mathrm{CP}$ & $\mathrm{CP}$ & $1.2 \mathrm{e}-04$ & $1.2 \mathrm{e}-05$ & no & 0.030 & 0.030 & 0.030 & 5.57721 & 0.00042 & 0.00042 & 2177.376 & 0.002 & 0.002 \\
\hline 206209135 & 2 & $\mathrm{CP}$ & $\mathrm{CP}$ & $8.4 \mathrm{e}-05$ & $1.1 \mathrm{e}-04$ & no & 0.032 & 0.031 & 0.029 & 15.18903 & 0.00315 & 0.00313 & 2156.465 & 0.005 & 0.00 \\
\hline 206209135 & 3 & $\mathrm{CP}$ & $\mathrm{CP}$ & $7.2 \mathrm{e}-04$ & $5.1 \mathrm{e}-04$ & no & 0.028 & 0.026 & 0.030 & 7.76018 & 0.00150 & 0.00150 & 2151.788 & 0.007 & 0.008 \\
\hline 206209135 & 4 & $\mathrm{CP}$ & $\mathrm{CP}$ & $8.4 \mathrm{e}-05$ & $1.1 \mathrm{e}-05$ & no & 0.036 & 0.038 & 0.034 & 24.15887 & 0.00385 & 0.00373 & 2154.054 & 0.005 & 0.005 \\
\hline 206312951 & 1 & $\mathrm{PC}$ & $\mathrm{PC}$ & $2.3 \mathrm{e}-02$ & $2.1 \mathrm{e}-02$ & $\ldots$ & 0.022 & 0.021 & 0.396 & 1.53402 & 0.00017 & 0.00018 & 2147.160 & 0.005 & 0.005 \\
\hline 206318379 & 1 & $\mathrm{PC}$ & $\mathrm{PC}$ & $1.0 \mathrm{e}-01$ & $4.6 \mathrm{e}-02$ & $\ldots$ & 0.075 & 0.076 & 0.076 & 2.26044 & 0.00003 & 0.00003 & 2147.250 & 0.000 & 0.000 \\
\hline 210448987 & 1 & $\mathrm{CP}$ & $\mathrm{CP}$ & $2.8 \mathrm{e}-07$ & $7.5 \mathrm{e}-08$ & no & 0.029 & 0.028 & 0.027 & 6.10233 & 0.00041 & 0.00039 & 2237.440 & 0.002 & 0.002 \\
\hline 210508766 & 1 & $\mathrm{CP}$ & $\mathrm{CP}$ & $5.1 \mathrm{e}-04$ & $3.9 \mathrm{e}-04$ & no & 0.029 & 0.028 & 0.028 & 2.74723 & 0.00013 & 0.00013 & 2234.060 & 0.002 & 0.002 \\
\hline 210508766 & 2 & $\mathrm{CP}$ & $\mathrm{CP}$ & $1.8 \mathrm{e}-02$ & $1.9 \mathrm{e}-02$ & no & 0.035 & 0.034 & 0.034 & 9.99744 & 0.00061 & 0.00062 & 2233.271 & 0.002 & 0.002 \\
\hline 210558622 & 1 & $\mathrm{PC}$ & $\mathrm{PC}$ & $2.2 \mathrm{e}-06$ & $2.1 \mathrm{e}-10$ & no & 0.035 & 0.033 & 0.033 & 19.56324 & 0.00237 & 0.00255 & 2250.779 & 0.003 & 0.003 \\
\hline 210564155 & 1 & UK & $\mathrm{PC}$ & $2.0 \mathrm{e}-02$ & $1.5 \mathrm{e}-02$ & $\ldots$ & 0.035 & 0.034 & 0.034 & 4.86407 & 0.00027 & 0.00028 & 2263.759 & 0.001 & 0.001 \\
\hline 210707130 & 1 & $\mathrm{CP}$ & $\mathrm{CP}$ & $1.4 \mathrm{e}-05$ & $3.4 \mathrm{e}-05$ & no & 0.019 & 0.019 & 0.019 & 0.68456 & 0.00002 & 0.00002 & 2232.367 & 0.001 & 0.001 \\
\hline 210750726 & 1 & $\mathrm{CP}$ & $\mathrm{CP}$ & $5.6 \mathrm{e}-04$ & $3.8 \mathrm{e}-04$ & no & 0.046 & 0.045 & 0.046 & 4.61228 & 0.00017 & 0.00017 & 2233.218 & 0.001 & 0.001 \\
\hline 210838726 & 1 & $\mathrm{CP}$ & $\mathrm{CP}$ & $9.8 \mathrm{e}-04$ & $8.1 \mathrm{e}-04$ & no & 0.020 & 0.018 & 0.020 & 1.09598 & 0.00006 & 0.00006 & 2233.008 & 0.002 & 0.002 \\
\hline 210968143 & 1 & $\mathrm{CP}$ & $\mathrm{CP}$ & $2.8 \mathrm{e}-13$ & $9.8 \mathrm{e}-12$ & no & 0.037 & 0.036 & 0.037 & 13.73491 & 0.00080 & 0.00080 & 2245.659 & 0.001 & 0.001 \\
\hline 210968143 & 2 & $\mathrm{CP}$ & $\mathrm{CP}$ & $2.6 \mathrm{e}-04$ & $1.5 \mathrm{e}-04$ & no & 0.019 & 0.017 & 0.017 & 2.90071 & 0.00032 & 0.00033 & 2233.740 & 0.004 & 0.004 \\
\hline 211077024 & 1 & $\mathrm{CP}$ & $\mathrm{CP}$ & $1.9 \mathrm{e}-05$ & $4.1 \mathrm{e}-06$ & no & 0.035 & 0.032 & 0.032 & 1.41960 & 0.00006 & 0.00006 & 2232.430 & 0.002 & 0.002 \\
\hline 211305568 & 1 & UK & $\mathrm{PC}$ & $5.6 \mathrm{e}-01$ & $1.0 \mathrm{e}+00$ & $\ldots$ & 0.041 & 0.041 & 0.038 & 11.55063 & 0.00112 & 0.00116 & 2336.344 & 0.002 & 0.002 \\
\hline $211305568^{\mathrm{e}}$ & 2 & UK & $\mathrm{PC}$ & $\ldots$ & $\ldots$ & $\ldots$ & 0.015 & 0.016 & 0.017 & 0.19785 & 0.00001 & 0.00001 & 2343.970 & 0.002 & 0.002 \\
\hline $211331236^{\mathrm{f}}$ & 1 & $\mathrm{PC}$ & $\mathrm{CP}$ & $4.7 \mathrm{e}-08$ & $2.8 \mathrm{e}-08$ & no & 0.037 & 0.037 & 0.036 & 1.29151 & 0.00004 & 0.00004 & 2309.776 & 0.001 & 0.001 \\
\hline $211331236^{\mathrm{g}}$ & 2 & UK & $\mathrm{CP}$ & $2.2 \mathrm{e}-06$ & $3.5 \mathrm{e}-06$ & no & 0.038 & 0.037 & 0.037 & 5.44482 & 0.00042 & 0.00040 & 2310.560 & 0.003 & 0.00 \\
\hline 211336288 & 1 & UK & $\mathrm{PC}$ & $1.9 \mathrm{e}-01$ & $1.2 \mathrm{e}-01$ & $\ldots$ & 0.018 & 0.015 & 0.017 & 0.22181 & 0.00002 & 0.00002 & 2344.090 & 0.002 & 0.002 \\
\hline 211357309 & 1 & $\mathrm{PC}$ & $\mathrm{PC}$ & $7.6 \mathrm{e}-02$ & $6.7 \mathrm{e}-02$ & $\ldots$ & 0.017 & 0.018 & 0.018 & 0.46394 & 0.00002 & 0.00002 & 2368.458 & 0.001 & 0.001 \\
\hline 211428897 & 1 & $\mathrm{PC}$ & $\mathrm{PC}$ & $7.0 \mathrm{e}-06$ & $5.5 \mathrm{e}-08$ & yes & 0.024 & 0.025 & 0.023 & 1.61093 & 0.00006 & 0.00006 & 2309.275 & 0.001 & 0.001 \\
\hline 211428897 & 2 & UK & $\mathrm{PC}$ & $7.4 \mathrm{e}-04$ & $2.5 \mathrm{e}-05$ & yes & 0.021 & 0.019 & 0.021 & 2.17807 & 0.00012 & 0.00012 & 2310.647 & 0.002 & 0.002 \\
\hline 211428897 & 3 & UK & $\mathrm{PC}$ & $1.2 \mathrm{e}-03$ & $5.2 \mathrm{e}-04$ & yes & 0.021 & 0.021 & 0.020 & 4.96883 & 0.00037 & 0.00037 & 2340.523 & 0.002 & 0.002 \\
\hline 211509553 & 1 & $\mathrm{PC}$ & $\mathrm{PC}$ & $1.0 \mathrm{e}-03$ & $1.3 \mathrm{e}-03$ & yes & 0.181 & 0.176 & 0.177 & 20.35954 & 0.00032 & 0.00033 & 2318.412 & 0.001 & 0.001 \\
\hline $211680698^{\mathrm{h}}$ & 1 & UK & $\mathrm{CP}$ & $5.6 \mathrm{e}-03$ & $7.6 \mathrm{e}-03$ & $\ldots$ & 0.032 & 0.029 & 0.031 & 50.92092 & 0.00525 & 0.00558 & 2327.473 & 0.004 & 0.004 \\
\hline 211694226 & 1 & UK & $\mathrm{PC}$ & $2.9 \mathrm{e}-01$ & $6.2 \mathrm{e}-01$ & yes & 0.020 & 0.017 & 0.368 & 1.91828 & 0.00017 & 0.00019 & 2342.946 & 0.002 & 0.002 \\
\hline 211762841 & 1 & UK & $\mathrm{PC}$ & $9.8 \mathrm{e}-02$ & $1.2 \mathrm{e}-01$ & $\ldots$ & 0.031 & 0.223 & 0.185 & 1.56494 & 0.00009 & 0.00009 & 2343.261 & 0.001 & 0.001 \\
\hline $211770795^{\mathrm{i}}$ & 1 & $\mathrm{PC}$ & $\mathrm{CP}$ & $1.2 \mathrm{e}-05$ & $7.6 \mathrm{e}-05$ & no & 0.031 & 0.031 & 0.029 & 7.72858 & 0.00070 & 0.00073 & 2315.826 & 0.003 & 0.00 \\
\hline $211791178^{j}$ & 1 & UK & $\mathrm{CP}$ & $1.7 \mathrm{e}-03$ & $1.5 \mathrm{e}-03$ & $\ldots$ & 0.028 & 0.028 & 0.029 & 9.56274 & 0.00067 & 0.00067 & 2342.604 & 0.002 & 0.002 \\
\hline 211799258 & 1 & UK & $\mathrm{PC}$ & $9.0 \mathrm{e}-01$ & $9.4 \mathrm{e}-01$ & no & 0.264 & 0.258 & 0.348 & 19.53406 & 0.00077 & 0.00079 & 2320.146 & 0.001 & 0.001 \\
\hline 211817229 & 1 & UK & $\mathrm{PC}$ & $3.9 \mathrm{e}-01$ & $9.5 \mathrm{e}-01$ & $\ldots$ & 0.276 & 0.266 & 0.176 & 2.17693 & 0.00005 & 0.00005 & 2342.525 & 0.001 & 0.001 \\
\hline $211818569^{\mathrm{k}}$ & 1 & $\mathrm{PC}$ & $\mathrm{CP}$ & $1.4 \mathrm{e}-04$ & $6.1 \mathrm{e}-04$ & no & 0.109 & 0.108 & 0.108 & 5.18575 & 0.00020 & 0.00020 & 2310.561 & 0.001 & 0.001 \\
\hline 211822797 & 1 & $\mathrm{CP}$ & $\mathrm{CP}$ & $6.7 \mathrm{e}-04$ & $7.6 \mathrm{e}-04$ & $\ldots$ & 0.032 & 0.030 & 0.029 & 21.16986 & 0.00169 & 0.00172 & 2332.577 & 0.002 & 0.002 \\
\hline 211826814 & 1 & UK & $\mathrm{PC}$ & $5.6 \mathrm{e}-01$ & $9.6 \mathrm{e}-01$ & $\ldots$ & 0.082 & 0.260 & 0.028 & 1.53453 & 0.00012 & 0.00013 & 2343.198 & 0.002 & 0.002 \\
\hline 211831378 & 1 & UK & FP & $2.4 \mathrm{e}-05$ & $1.9 \mathrm{e}-06$ & no & 0.015 & 0.074 & 0.579 & 3.48929 & 0.00050 & 0.00048 & 2310.755 & 0.005 & 0.005 \\
\hline $211924657^{1}$ & 1 & PC & $\mathrm{PC}$ & $5.3 \mathrm{e}-01$ & $6.4 \mathrm{e}-01$ & no & 0.052 & 0.051 & 0.051 & 2.64484 & 0.00011 & 0.00011 & 2311.641 & 0.001 & 0.001 \\
\hline 211965883 & 1 & $\mathrm{PC}$ & $\mathrm{PC}$ & $3.4 \mathrm{e}-01$ & $3.8 \mathrm{e}-01$ & $\ldots$ & 0.043 & 0.039 & 0.039 & 10.55630 & 0.00066 & 0.00064 & 2334.605 & 0.001 & 0.001 \\
\hline 211969807 & 1 & $\mathrm{PC}$ & $\mathrm{PC}$ & $8.6 \mathrm{e}-02$ & $9.5 \mathrm{e}-02$ & $\ldots$ & 0.037 & 0.036 & 0.037 & 1.97424 & 0.00011 & 0.00011 & 2342.915 & 0.001 & 0.001 \\
\hline
\end{tabular}


Table 3

(Continued)

\begin{tabular}{|c|c|c|c|c|c|c|c|c|c|c|c|c|c|c|c|}
\hline \multirow[b]{2}{*}{ EPIC } & \multirow[b]{2}{*}{ K2OI } & \multicolumn{2}{|c|}{ Disposition } & \multicolumn{2}{|c|}{ VESPA FPP ${ }^{a}$} & \multirow{2}{*}{$\begin{array}{c}\text { Nearby } \\
\text { Star? }^{b}\end{array}$} & \multicolumn{3}{|c|}{$R_{p} / R_{\star}$} & \multicolumn{3}{|c|}{$P$ (days) $)^{c}$} & \multicolumn{3}{|c|}{$t 0(\mathrm{BKJD})^{\mathrm{c}}$} \\
\hline & & Old & New & Small $R_{p}$ & $\operatorname{Big} R_{p}$ & & K2SFF & k2phot & $\mathrm{K} 2 \mathrm{SC}$ & Val & - Err & + Err & Val & -Err & + Err \\
\hline 211970234 & 1 & UK & FP & $1.6 \mathrm{e}-01$ & $1.9 \mathrm{e}-01$ & no & 0.069 & 0.104 & 0.212 & 1.48350 & 0.00004 & 0.00003 & 2310.371 & 0.001 & 0.001 \\
\hline 211988320 & 1 & UK & PC & $7.5 \mathrm{e}-02$ & $7.2 \mathrm{e}-02$ & $\ldots$ & 0.041 & 0.036 & 0.034 & 63.84808 & 0.00588 & 0.00560 & 2309.721 & 0.004 & 0.005 \\
\hline $212006344^{\mathrm{m}}$ & 1 & $\mathrm{PC}$ & $\mathrm{CP}$ & $1.5 \mathrm{e}-04$ & $1.7 \mathrm{e}-04$ & no & 0.020 & 0.019 & 0.020 & 2.21940 & 0.00007 & 0.00007 & 2311.048 & 0.001 & 0.001 \\
\hline $212069861^{\mathrm{n}}$ & 1 & $\mathrm{PC}$ & $\mathrm{CP}$ & $1.1 \mathrm{e}-04$ & $2.9 \mathrm{e}-06$ & no & 0.043 & 0.042 & 0.040 & 30.95676 & 0.00272 & 0.00259 & 2314.492 & 0.003 & 0.003 \\
\hline $212154564^{\circ}$ & 1 & $\mathrm{PC}$ & $\mathrm{CP}$ & $6.8 \mathrm{e}-07$ & $9.7 \mathrm{e}-07$ & no & 0.070 & 0.067 & 0.069 & 6.41354 & 0.00026 & 0.00025 & 2309.183 & 0.002 & 0.002 \\
\hline $212398486^{\mathrm{P}}$ & 1 & UK & $\mathrm{CP}$ & $7.5 \mathrm{e}-03$ & $1.7 \mathrm{e}-03$ & $\ldots$ & 0.050 & 0.050 & 0.045 & 21.75026 & 0.00196 & 0.00199 & 2410.088 & 0.002 & 0.002 \\
\hline 212443973 & 1 & $\mathrm{PC}$ & $\mathrm{PC}$ & $1.6 \mathrm{e}-01$ & $8.9 \mathrm{e}-01$ & $\ldots$ & 0.024 & 0.295 & 0.096 & 0.77923 & 0.00005 & 0.00006 & 2423.710 & 0.002 & 0.002 \\
\hline $212460519^{\mathrm{q}}$ & 1 & $\mathrm{PC}$ & $\mathrm{CP}$ & $4.2 \mathrm{e}-13$ & $1.1 \mathrm{e}-11$ & no & 0.027 & 0.028 & 0.027 & 7.38707 & 0.00025 & 0.00025 & 2390.794 & 0.001 & 0.001 \\
\hline $212554013^{\mathrm{r}}$ & 1 & $\mathrm{PC}$ & $\mathrm{CP}$ & $1.0 \mathrm{e}-03$ & $1.6 \mathrm{e}-03$ & no & 0.117 & 0.115 & 0.117 & 3.58816 & 0.00002 & 0.00002 & 2390.926 & 0.000 & 0.000 \\
\hline 212572452 & 1 & $\mathrm{PC}$ & FP & $3.3 \mathrm{e}-07$ & $4.3 \mathrm{e}-08$ & no & 0.072 & 0.067 & 0.176 & 2.58148 & 0.00001 & 0.00001 & 2390.028 & 0.000 & 0.000 \\
\hline 212628098 & 1 & FP & FP & $9.2 \mathrm{e}-02$ & 3.0e- -02 & yes & 0.228 & 0.278 & 0.286 & 4.35244 & 0.00003 & 0.00003 & 2390.348 & 0.000 & 0.000 \\
\hline 212634172 & 1 & UK & PC & $3.0 \mathrm{e}-01$ & $1.0 \mathrm{e}+00$ & $\ldots$ & 0.093 & 0.081 & 0.069 & 2.85177 & 0.00009 & 0.00010 & 2421.669 & 0.001 & 0.001 \\
\hline 212679181 & 1 & $\mathrm{PC}$ & PC & $4.2 \mathrm{e}-04$ & $6.4 \mathrm{e}-04$ & yes & 0.026 & 0.024 & 0.026 & 1.05459 & 0.00002 & 0.00001 & 2423.570 & 0.000 & 0.000 \\
\hline 212679798 & 1 & UK & FP & $2.1 \mathrm{e}-01$ & $9.5 \mathrm{e}-01$ & yes & 0.482 & 0.678 & 0.158 & 1.83473 & 0.00002 & 0.00002 & 2389.389 & 0.002 & 0.002 \\
\hline $212686205^{\mathrm{s}}$ & 1 & UK & $\mathrm{CP}$ & $1.1 \mathrm{e}-07$ & $4.2 \mathrm{e}-06$ & no & 0.017 & 0.016 & 0.014 & 5.67582 & 0.00041 & 0.00041 & 2422.503 & 0.002 & 0.002 \\
\hline 212690867 & 1 & UK & $\mathrm{PC}$ & $2.9 \mathrm{e}-02$ & $3.5 \mathrm{e}-02$ & $\ldots$ & 0.049 & 0.047 & 0.049 & 25.86125 & 0.00309 & 0.00298 & 2422.464 & 0.003 & 0.003 \\
\hline 212773272 & 1 & UK & FP & $9.4 \mathrm{e}-01$ & $9.8 \mathrm{e}-01$ & no & 0.623 & 0.203 & 0.205 & 4.68189 & 0.00005 & 0.00005 & 2389.666 & 0.001 & 0.001 \\
\hline 212773309 & 1 & FP & FP & $8.5 \mathrm{e}-01$ & $9.4 \mathrm{e}-01$ & yes & 0.732 & 0.738 & 0.728 & 4.68199 & 0.00007 & 0.00006 & 2389.665 & 0.001 & 0.001 \\
\hline 213951550 & 1 & UK & FP & $1.0 \mathrm{e}+00$ & $1.0 \mathrm{e}+00$ & yes & 0.683 & 0.625 & $\ldots$ & 1.11704 & 0.00002 & 0.00002 & 2478.230 & 0.001 & 0.001 \\
\hline 214254518 & 1 & UK & $\mathrm{PC}$ & $9.8 \mathrm{e}-03$ & $1.2 \mathrm{e}-02$ & $\ldots$ & 0.015 & 0.015 & $\ldots$ & 5.05899 & 0.00054 & 0.00053 & 2506.630 & 0.003 & 0.003 \\
\hline 214522613 & 1 & UK & $\mathrm{PC}$ & $5.1 \mathrm{e}-02$ & $4.9 \mathrm{e}-02$ & $\ldots$ & 0.024 & 0.022 & $\ldots$ & 10.99041 & 0.00126 & 0.00124 & 2506.314 & 0.002 & 0.002 \\
\hline $214787262^{t}$ & 1 & UK & $\mathrm{CP}$ & $6.1 \mathrm{e}-04$ & $1.4 \mathrm{e}-03$ & $\ldots$ & 0.027 & 0.026 & $\ldots$ & 8.23949 & 0.00029 & 0.00029 & 2502.009 & 0.001 & 0.001 \\
\hline 216892056 & 1 & UK & $\mathrm{PC}$ & $6.1 \mathrm{e}-01$ & $9.8 \mathrm{e}-01$ & no & 0.279 & 0.083 & $\ldots$ & 2.78592 & 0.00005 & 0.00005 & 2478.406 & 0.001 & 0.001 \\
\hline $217941732^{\mathrm{u}}$ & 1 & UK & $\mathrm{CP}$ & $1.2 \mathrm{e}-06$ & $2.9 \mathrm{e}-07$ & no & 0.015 & 0.015 & $\ldots$ & 2.49412 & 0.00013 & 0.00013 & 2507.612 & 0.001 & 0.001 \\
\hline
\end{tabular}

Notes.

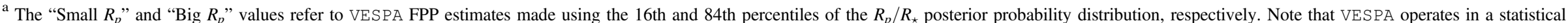
fashion and that the reported FPP may occasionally be higher for the small $R_{p}$ case than for the big $R_{p}$ case due to changes in the simulated population of stars.

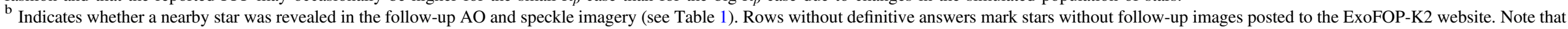
planet radius estimates are not corrected for flux dilution due to the presence of nearby stars.

${ }^{c}$ Values and errors for orbital period and transit center are from fits using K2SFF photometry.

d Now validated as K2-116b.

e VESPA was unable to calculate an FPP for EPIC 211305568.02. See Section 7.2.5.

${ }^{\mathrm{f}}$ Now validated as K2-117b.

g Now validated as $\mathrm{K} 2-117 \mathrm{c}$

${ }^{\mathrm{h}}$ Now validated as K2-118b.

${ }^{\mathrm{i}}$ Now validated as K2-119b.

${ }_{\mathrm{j}}^{\mathrm{j}}$ Now validated as K2-120b

${ }^{\mathrm{k}}$ Now validated as K2-121b.

${ }^{1}$ This K2OI displays transit timing variations, but our fits assumed a linear ephemeris.

$\mathrm{m}$ Now validated as $\mathrm{K} 2-122 \mathrm{~b}$.

${ }^{\mathrm{n}}$ Now validated as $\mathrm{K} 2-123 \mathrm{~b}$

${ }^{\mathrm{o}}$ Now validated as K2-124b.

${ }^{\mathrm{P}}$ Now validated as $\mathrm{K} 2-125 \mathrm{~b}$

${ }^{\mathrm{q}}$ Now validated as K2-126b.

${ }^{\mathrm{r}}$ Now validated as K2-127b.

${ }^{s}$ Now validated as K2-128b.

${ }^{\mathrm{t}}$ Now validated as K2-129b.

${ }^{\mathrm{u}}$ Now validated as K2-130b. 
Table 4

\begin{tabular}{|c|c|c|c|c|c|c|c|c|c|c|c|c|c|c|c|c|c|c|}
\hline \multirow[b]{2}{*}{ EPIC } & \multirow[b]{2}{*}{ K2OI } & \multirow{2}{*}{$\begin{array}{c}P^{\mathrm{b}} \\
\text { (days) }\end{array}$} & \multicolumn{3}{|c|}{$R_{p} / R_{\star}$} & \multirow[b]{2}{*}{$a / \mathrm{R}_{\star}$} & \multirow[b]{2}{*}{$i$} & \multicolumn{2}{|c|}{$\begin{array}{c}\text { Limb } \\
\text { Darkening }\end{array}$} & \multirow[b]{2}{*}{$e$} & \multirow[b]{2}{*}{$\omega$} & \multicolumn{3}{|c|}{$R_{p}\left(R_{\oplus}\right)$} & \multirow{2}{*}{$\begin{array}{c}a \\
\text { (au) }\end{array}$} & \multicolumn{3}{|c|}{$F_{p}\left(F_{\oplus}\right)$} \\
\hline & & & Val & - Err & + Err & & & $q_{1}$ & $q_{2}$ & & & Val & - Err & + Err & & Val & - Err & + Err \\
\hline 201205469 & 1 & 3.47134 & 0.074 & 0.002 & 0.002 & 13.51 & 88.90 & 0.48 & 0.30 & 0.07 & -25.11 & 4.75 & 0.33 & 0.34 & 0.038 & 46.4 & 16.3 & 23.0 \\
\hline 201208431 & 1 & 10.00342 & 0.034 & 0.001 & 0.001 & 27.68 & 89.54 & 0.45 & 0.33 & 0.08 & -41.44 & 2.10 & 0.19 & 0.20 & 0.078 & 15.6 & 6.2 & 9.2 \\
\hline 201345483 & 1 & 1.72926 & 0.140 & 0.002 & 0.003 & 8.02 & 87.75 & 0.45 & 0.43 & 0.10 & 59.61 & 10.44 & 0.70 & 0.90 & 0.025 & 380.5 & 150.1 & 219.7 \\
\hline 201549860 & 1 & 5.60835 & 0.028 & 0.001 & 0.001 & 19.10 & 88.58 & 0.55 & 0.44 & 0.12 & 41.16 & 1.93 & 0.11 & 0.13 & 0.055 & 68.0 & 10.5 & 12.1 \\
\hline 201549860 & 2 & 2.39996 & 0.019 & 0.001 & 0.001 & 10.53 & 88.98 & 0.54 & 0.45 & 0.13 & -74.55 & 1.32 & 0.08 & 0.08 & 0.031 & 211.0 & 32.5 & 37.4 \\
\hline 201617985 & 1 & 7.28118 & 0.033 & 0.002 & 0.003 & 26.16 & 88.30 & 0.47 & 0.27 & 0.23 & 62.91 & 1.77 & 0.17 & 0.21 & 0.060 & 9.2 & 2.6 & 3.3 \\
\hline 201635569 & 1 & 8.36879 & 0.110 & 0.004 & 0.004 & 22.94 & 88.19 & 0.50 & 0.33 & 0.14 & -70.72 & 7.49 & 0.47 & 0.48 & 0.069 & 5.5 & 3.2 & 6.1 \\
\hline 201637175 & 1 & 0.38108 & 0.075 & 0.004 & 0.004 & 3.19 & 77.48 & 0.48 & 0.30 & 0.19 & 46.70 & 4.75 & 0.36 & 0.35 & 0.009 & 737.9 & 196.7 & 242.8 \\
\hline 201717274 & 1 & 3.52675 & 0.038 & 0.002 & 0.004 & 15.20 & 88.23 & 0.59 & 0.29 & 0.13 & 23.29 & 1.32 & 0.25 & 0.26 & 0.026 & 15.0 & 3.3 & 4.1 \\
\hline 201855371 & 1 & 17.96903 & 0.030 & 0.002 & 0.002 & 40.17 & 89.08 & 0.51 & 0.37 & 0.18 & 52.06 & 2.03 & 0.16 & 0.18 & 0.117 & 10.5 & 2.9 & 3.7 \\
\hline 205924614 & 1 & 2.84927 & 0.056 & 0.001 & 0.002 & 10.46 & 88.21 & 0.53 & 0.44 & 0.07 & 23.94 & 4.38 & 0.25 & 0.29 & 0.035 & 141.3 & 23.5 & 28.8 \\
\hline 206011691 & 1 & 9.32504 & 0.026 & 0.001 & 0.001 & 25.37 & 88.98 & 0.52 & 0.41 & 0.10 & 34.48 & 1.84 & 0.10 & 0.10 & 0.076 & 10.0 & 1.7 & 2.1 \\
\hline 206011691 & 2 & 15.50192 & 0.035 & 0.002 & 0.002 & 35.50 & 88.85 & 0.50 & 0.41 & 0.21 & 59.96 & 2.49 & 0.19 & 0.17 & 0.107 & 5.1 & 0.9 & 1.1 \\
\hline 206119924 & 1 & 4.65541 & 0.009 & 0.000 & 0.000 & 15.37 & 89.09 & 0.54 & 0.44 & 0.06 & -23.44 & 0.69 & 0.04 & 0.04 & 0.048 & 78.6 & 10.5 & 12.2 \\
\hline 206209135 & 1 & 5.57721 & 0.030 & 0.001 & 0.002 & 24.16 & 89.15 & 0.54 & 0.27 & 0.11 & 7.49 & 1.08 & 0.11 & 0.11 & 0.040 & 8.5 & 1.1 & 1.2 \\
\hline 206209135 & 2 & 15.18903 & 0.032 & 0.002 & 0.002 & 47.82 & 89.54 & 0.54 & 0.27 & 0.11 & 16.83 & 1.16 & 0.13 & 0.13 & 0.078 & 2.2 & 0.3 & 0.3 \\
\hline 206209135 & 3 & 7.76018 & 0.028 & 0.002 & 0.002 & 29.91 & 89.26 & 0.54 & 0.27 & 0.11 & 14.28 & 1.01 & 0.12 & 0.12 & 0.050 & 5.4 & 0.7 & 0.8 \\
\hline 206209135 & 4 & 24.15887 & 0.036 & 0.002 & 0.002 & 64.53 & 89.68 & 0.54 & 0.27 & 0.11 & 11.39 & 1.29 & 0.13 & 0.14 & 0.106 & 1.2 & 0.2 & 0.2 \\
\hline 206312951 & 1 & 1.53402 & 0.022 & 0.002 & 0.002 & 9.41 & 87.23 & 0.47 & 0.27 & 0.11 & 32.40 & 1.13 & 0.10 & 0.11 & 0.021 & 120.2 & 16.8 & 19.0 \\
\hline 206318379 & 1 & 2.26044 & 0.075 & 0.002 & 0.004 & 16.21 & 88.35 & 0.54 & 0.27 & 0.13 & 42.80 & 2.30 & 0.26 & 0.28 & 0.020 & 30.1 & 3.8 & 4.5 \\
\hline 210448987 & 1 & 6.10233 & 0.029 & 0.001 & 0.001 & 19.77 & 89.29 & 0.54 & 0.45 & 0.06 & -27.89 & 1.97 & 0.11 & 0.13 & 0.059 & 62.9 & 8.4 & 9.2 \\
\hline 210508766 & 1 & 2.74723 & 0.029 & 0.001 & 0.001 & 12.59 & 88.72 & 0.46 & 0.29 & 0.07 & -5.43 & 1.71 & 0.10 & 0.10 & 0.032 & 38.8 & 5.9 & 6.3 \\
\hline 210508766 & 2 & 9.99744 & 0.035 & 0.001 & 0.001 & 29.67 & 89.51 & 0.47 & 0.29 & 0.06 & -19.35 & 2.11 & 0.12 & 0.12 & 0.076 & 6.9 & 1.0 & 1.1 \\
\hline 210558622 & 1 & 19.56324 & 0.035 & 0.001 & 0.001 & 38.50 & 89.75 & 0.53 & 0.43 & 0.48 & -90.15 & 2.59 & 0.15 & 0.17 & 0.125 & 13.2 & 2.1 & 2.3 \\
\hline 210564155 & 1 & 4.86407 & 0.035 & 0.002 & 0.002 & 26.74 & 89.00 & 0.53 & 0.26 & 0.13 & 36.10 & 1.09 & 0.13 & 0.13 & 0.036 & 7.7 & 1.0 & 1.2 \\
\hline 210707130 & 1 & 0.68456 & 0.019 & 0.001 & 0.001 & 4.30 & 80.21 & 0.56 & 0.45 & 0.24 & 67.59 & 1.37 & 0.11 & 0.11 & 0.013 & 1069.1 & 144.7 & 163.5 \\
\hline 210750726 & 1 & 4.61228 & 0.046 & 0.003 & 0.003 & 19.98 & 87.75 & 0.49 & 0.27 & 0.26 & 72.02 & 2.30 & 0.21 & 0.23 & 0.042 & 16.4 & 2.0 & 2.2 \\
\hline 210838726 & 1 & 1.09598 & 0.020 & 0.001 & 0.001 & 7.36 & 85.77 & 0.47 & 0.28 & 0.15 & 51.29 & 1.08 & 0.08 & 0.09 & 0.017 & 144.4 & 18.0 & 20.2 \\
\hline 210968143 & 1 & 13.73491 & 0.037 & 0.001 & 0.001 & 33.90 & 89.40 & 0.54 & 0.45 & 0.07 & 16.40 & 2.53 & 0.13 & 0.14 & 0.100 & 10.2 & 1.4 & 1.7 \\
\hline 210968143 & 2 & 2.90071 & 0.019 & 0.001 & 0.002 & 12.02 & 86.94 & 0.55 & 0.45 & 0.19 & 56.76 & 1.30 & 0.11 & 0.13 & 0.035 & 80.8 & 11.1 & 13.2 \\
\hline 211077024 & 1 & 1.41960 & 0.035 & 0.001 & 0.001 & 11.19 & 88.62 & 0.43 & 0.25 & 0.09 & -23.36 & 1.22 & 0.12 & 0.12 & 0.018 & 56.1 & 6.6 & 7.4 \\
\hline 211305568 & 1 & 11.55063 & 0.041 & 0.004 & 0.805 & 36.12 & 88.64 & 0.49 & 0.26 & 0.35 & 10.33 & 1.99 & 0.25 & 39.17 & 0.078 & 5.7 & 0.7 & 0.8 \\
\hline 211305568 & 2 & 0.19785 & 0.015 & 0.001 & 0.002 & 2.50 & 79.26 & 0.48 & 0.26 & 0.13 & 33.20 & 0.72 & 0.08 & 0.11 & 0.005 & 1292.5 & 158.3 & 178.2 \\
\hline 211331236 & 1 & 1.29151 & 0.037 & 0.001 & 0.001 & 8.32 & 88.36 & 0.46 & 0.28 & 0.06 & -22.63 & 1.96 & 0.12 & 0.12 & 0.019 & 145.8 & 18.9 & 21.5 \\
\hline 211331236 & 2 & 5.44482 & 0.038 & 0.001 & 0.001 & 20.93 & 89.49 & 0.46 & 0.28 & 0.15 & -77.06 & 2.03 & 0.13 & 0.13 & 0.051 & 21.4 & 2.8 & 3.2 \\
\hline 211336288 & 1 & 0.22181 & 0.018 & 0.002 & 0.002 & 2.28 & 77.48 & 0.47 & 0.33 & 0.17 & 54.63 & 1.14 & 0.13 & 0.15 & 0.006 & 1140.9 & 151.6 & 172.3 \\
\hline 211357309 & 1 & 0.46394 & 0.017 & 0.001 & 0.001 & 4.28 & 86.80 & 0.47 & 0.27 & 0.08 & -36.30 & 0.84 & 0.06 & 0.06 & 0.010 & 437.3 & 56.8 & 63.9 \\
\hline 211428897 & 1 & 1.61093 & 0.024 & 0.001 & 0.001 & 12.64 & 89.08 & 0.47 & 0.25 & 0.15 & -71.99 & 0.75 & 0.08 & 0.08 & 0.021 & 48.0 & 5.9 & 6.8 \\
\hline 211428897 & 2 & 2.17807 & 0.021 & 0.001 & 0.001 & 16.36 & 89.21 & 0.47 & 0.25 & 0.11 & -54.04 & 0.65 & 0.07 & 0.07 & 0.025 & 32.1 & 3.9 & 4.6 \\
\hline 211428897 & 3 & 4.96883 & 0.021 & 0.001 & 0.001 & 29.12 & 89.52 & 0.47 & 0.25 & 0.10 & -40.14 & 0.67 & 0.08 & 0.08 & 0.044 & 10.7 & 1.3 & 1.5 \\
\hline 211509553 & 1 & 20.35954 & 0.181 & 0.002 & 0.003 & 47.46 & 89.61 & 0.48 & 0.28 & 0.09 & 43.86 & 10.82 & 0.58 & 0.59 & 0.119 & 1.8 & 0.3 & 0.4 \\
\hline 211680698 & 1 & 50.92092 & 0.032 & 0.002 & 0.002 & 71.82 & 89.50 & 0.54 & 0.45 & 0.20 & 58.67 & 2.54 & 0.20 & 0.23 & 0.245 & 4.3 & 0.6 & 0.6 \\
\hline 211694226 & 1 & 1.91828 & 0.020 & 0.002 & 0.002 & 10.51 & 86.33 & 0.49 & 0.26 & 0.19 & 59.28 & 0.99 & 0.11 & 0.13 & 0.021 & 75.8 & 12.1 & 13.8 \\
\hline 211762841 & 1 & 1.56494 & 0.031 & 0.004 & 0.007 & 7.91 & 83.60 & 0.51 & 0.27 & 0.17 & 55.97 & 2.14 & 0.30 & 0.49 & 0.023 & 157.6 & 25.9 & 29.7 \\
\hline 211770795 & 1 & 7.72858 & 0.031 & 0.001 & 0.001 & 21.58 & 89.40 & 0.51 & 0.27 & 0.07 & -37.71 & 2.29 & 0.13 & 0.15 & 0.070 & 54.9 & 8.8 & 9.6 \\
\hline 211791178 & 1 & 9.56274 & 0.028 & 0.001 & 0.001 & 24.05 & 89.57 & 0.49 & 0.38 & 0.14 & -81.19 & 2.01 & 0.12 & 0.13 & 0.078 & 35.1 & 5.1 & 5.9 \\
\hline
\end{tabular}


Table 4

Continued)

\begin{tabular}{|c|c|c|c|c|c|c|c|c|c|c|c|c|c|c|c|c|c|c|}
\hline \multirow[b]{2}{*}{ EPIC } & \multirow[b]{2}{*}{ K2OI } & \multirow{2}{*}{$\begin{array}{c}P^{\mathrm{b}} \\
\text { (days) }\end{array}$} & \multicolumn{3}{|c|}{$R_{p} / R_{\star}$} & \multirow[b]{2}{*}{$a / \mathrm{R}_{\star}$} & \multirow[b]{2}{*}{$i$} & \multicolumn{2}{|c|}{$\begin{array}{c}\text { Limb } \\
\text { Darkening }\end{array}$} & \multirow[b]{2}{*}{$e$} & \multirow[b]{2}{*}{$\omega$} & \multicolumn{3}{|c|}{$R_{p}\left(R_{\oplus}\right)$} & \multirow{2}{*}{$\begin{array}{c}a \\
(\mathrm{au})\end{array}$} & \multicolumn{3}{|c|}{$F_{p}\left(F_{\oplus}\right)$} \\
\hline & & & Val & - Err & + Err & & & $q_{1}$ & $q_{2}$ & & & Val & - Err & + Err & & Val & - Err & + Err \\
\hline 211799258 & 1 & 19.53406 & 0.264 & 0.021 & 0.168 & 77.55 & 89.26 & 0.54 & 0.45 & 0.48 & 89.44 & 9.46 & 1.93 & 6.33 & 0.087 & 1.0 & 0.6 & 1.4 \\
\hline 211817229 & 1 & 2.17693 & 0.276 & 0.208 & 0.494 & 17.03 & 85.82 & 0.55 & 0.44 & 0.08 & -4.12 & 7.13 & 5.51 & 12.83 & 0.019 & 15.1 & 11.9 & 56.6 \\
\hline 211818569 & 1 & 5.18575 & 0.109 & 0.005 & 0.005 & 14.71 & 87.25 & 0.50 & 0.27 & 0.22 & 72.97 & 9.12 & 0.68 & 0.66 & 0.052 & 89.5 & 11.2 & 12.5 \\
\hline 211822797 & 1 & 21.16986 & 0.032 & 0.001 & 0.001 & 48.67 & 89.73 & 0.45 & 0.25 & 0.06 & -21.51 & 1.98 & 0.11 & 0.11 & 0.131 & 3.5 & 0.5 & 0.5 \\
\hline 211826814 & 1 & 1.53453 & 0.082 & 0.056 & 0.520 & 12.15 & 85.00 & 0.56 & 0.47 & 0.09 & -8.50 & 2.34 & 1.65 & 14.85 & 0.015 & 26.0 & 18.5 & 63.9 \\
\hline 211831378 & 1 & 3.48929 & 0.015 & 0.001 & 0.001 & 14.01 & 88.89 & 0.49 & 0.37 & 0.08 & -22.01 & 0.91 & 0.07 & 0.07 & 0.037 & 24.6 & 7.1 & 10.5 \\
\hline 211924657 & $1^{\mathrm{c}}$ & 2.64484 & 0.052 & 0.001 & 0.002 & 13.85 & 88.77 & 0.48 & 0.28 & 0.10 & -26.38 & 1.83 & 0.21 & 0.24 & 0.026 & 18.8 & 2.6 & 2.9 \\
\hline 211965883 & 1 & 10.55630 & 0.043 & 0.004 & 0.004 & 29.73 & 88.32 & 0.43 & 0.25 & 0.23 & 62.02 & 2.79 & 0.30 & 0.27 & 0.083 & 11.4 & 1.5 & 1.7 \\
\hline 211969807 & 1 & 1.97424 & 0.037 & 0.001 & 0.002 & 9.93 & 88.00 & 0.51 & 0.26 & 0.09 & 6.24 & 1.96 & 0.15 & 0.15 & 0.023 & 62.1 & 13.8 & 16.0 \\
\hline 211970234 & 1 & 1.48350 & 0.069 & 0.003 & 0.015 & 12.68 & 87.70 & 0.51 & 0.40 & 0.12 & 10.96 & 1.42 & 0.30 & 0.41 & 0.015 & 19.2 & 4.3 & 5.0 \\
\hline 211988320 & 1 & 63.84808 & 0.041 & 0.001 & 0.001 & 92.33 & 89.80 & 0.50 & 0.27 & 0.07 & 3.10 & 2.86 & 0.15 & 0.16 & 0.276 & 0.9 & 0.1 & 0.1 \\
\hline 212006344 & 1 & 2.21940 & 0.020 & 0.001 & 0.001 & 10.42 & 86.39 & 0.46 & 0.25 & 0.21 & 60.80 & 1.28 & 0.08 & 0.08 & 0.029 & 79.9 & 11.1 & 13.1 \\
\hline 212069861 & 1 & 30.95676 & 0.043 & 0.001 & 0.001 & 61.89 & 89.79 & 0.53 & 0.42 & 0.06 & -33.53 & 2.65 & 0.15 & 0.15 & 0.167 & 2.9 & 0.4 & 0.5 \\
\hline 212154564 & 1 & 6.41354 & 0.070 & 0.002 & 0.002 & 30.09 & 89.51 & 0.47 & 0.33 & 0.08 & -26.80 & 2.64 & 0.24 & 0.24 & 0.051 & 8.7 & 1.1 & 1.2 \\
\hline 212398486 & 1 & 21.75026 & 0.050 & 0.002 & 0.002 & 63.31 & 89.72 & 0.45 & 0.25 & 0.08 & -3.74 & 2.18 & 0.18 & 0.19 & 0.121 & 2.0 & 0.3 & 0.3 \\
\hline 212443973 & 1 & 0.77923 & 0.024 & 0.005 & 0.183 & 7.18 & 83.37 & 0.48 & 0.26 & 0.12 & 23.59 & 0.90 & 0.19 & 6.84 & 0.011 & 98.7 & 11.5 & 13.0 \\
\hline 212460519 & 1 & 7.38707 & 0.027 & 0.000 & 0.000 & 22.38 & 89.40 & 0.47 & 0.26 & 0.06 & -33.72 & 1.84 & 0.10 & 0.11 & 0.066 & 35.3 & 5.9 & 6.6 \\
\hline 212554013 & 1 & 3.58816 & 0.117 & 0.002 & 0.002 & 12.02 & 87.22 & 0.55 & 0.27 & 0.12 & -65.74 & 8.66 & 0.59 & 0.68 & 0.041 & 105.6 & 17.8 & 20.7 \\
\hline 212572452 & 1 & 2.58148 & 0.072 & 0.003 & 0.003 & 9.92 & 86.28 & 0.58 & 0.45 & 0.15 & -76.47 & 5.30 & 0.37 & 0.40 & 0.033 & 132.0 & 33.7 & 41.8 \\
\hline 212628098 & 1 & 4.35244 & 0.228 & 0.006 & 0.006 & 17.15 & 87.29 & 0.55 & 0.42 & 0.18 & 91.75 & 14.06 & 0.78 & 0.78 & 0.044 & 81.2 & 11.7 & 13.1 \\
\hline 212634172 & 1 & 2.85177 & 0.093 & 0.028 & 0.528 & 14.47 & 86.15 & 0.56 & 0.44 & 0.12 & 6.67 & 3.51 & 1.12 & 20.03 & 0.027 & 18.8 & 2.6 & 2.9 \\
\hline 212679181 & 1 & 1.05459 & 0.026 & 0.003 & 0.003 & 8.02 & 83.74 & 0.47 & 0.31 & 0.18 & 64.17 & 1.25 & 0.18 & 0.15 & 0.016 & 114.7 & 13.9 & 16.5 \\
\hline 212679798 & 1 & 1.83473 & 0.482 & 0.231 & 0.335 & 8.98 & 84.65 & 0.53 & 0.26 & 0.52 & -91.97 & 29.52 & 14.20 & 20.58 & 0.024 & 164.7 & 28.0 & 35.0 \\
\hline 212686205 & 1 & 5.67582 & 0.017 & 0.001 & 0.001 & 15.46 & 87.40 & 0.48 & 0.26 & 0.23 & 62.55 & 1.42 & 0.13 & 0.16 & 0.056 & 68.8 & 9.7 & 11.1 \\
\hline 212690867 & 1 & 25.86125 & 0.049 & 0.001 & 0.002 & 64.68 & 89.76 & 0.48 & 0.29 & 0.09 & -26.75 & 2.20 & 0.18 & 0.19 & 0.133 & 1.4 & 0.2 & 0.3 \\
\hline 212773272 & 1 & 4.68189 & 0.623 & 0.281 & 0.259 & 15.60 & 85.55 & 0.56 & 0.47 & 0.07 & -36.21 & 29.05 & 13.26 & 12.25 & 0.036 & 13.8 & 2.0 & 2.4 \\
\hline 212773309 & 1 & 4.68199 & 0.732 & 0.348 & 0.201 & 16.74 & 85.66 & 0.48 & 0.26 & 0.14 & -79.60 & 46.96 & 22.42 & 13.10 & 0.048 & 69.5 & 8.4 & 9.8 \\
\hline 213951550 & 1 & 1.11704 & 0.683 & 0.314 & 0.229 & 6.71 & 79.52 & 0.57 & 0.28 & 0.08 & -44.84 & 35.08 & 16.27 & 11.98 & 0.016 & 166.0 & 26.5 & 31.7 \\
\hline 214254518 & 1 & 5.05899 & 0.015 & 0.001 & 0.001 & 16.18 & 89.17 & 0.50 & 0.38 & 0.07 & -30.08 & 1.12 & 0.07 & 0.08 & 0.051 & 56.1 & 7.9 & 9.2 \\
\hline 214522613 & 1 & 10.99041 & 0.024 & 0.002 & 0.002 & 36.38 & 88.90 & 0.49 & 0.26 & 0.22 & 63.16 & 1.15 & 0.12 & 0.13 & 0.075 & 6.9 & 1.2 & 1.4 \\
\hline 214787262 & 1 & 8.23949 & 0.027 & 0.001 & 0.001 & 34.37 & 89.21 & 0.49 & 0.27 & 0.13 & 39.89 & 1.04 & 0.09 & 0.10 & 0.057 & 4.5 & 0.5 & 0.6 \\
\hline 216892056 & 1 & 2.78592 & 0.279 & 0.232 & 0.412 & 15.87 & 85.50 & 0.55 & 0.44 & 0.06 & 4.45 & 12.11 & 10.13 & 17.91 & 0.028 & 25.5 & 3.1 & 3.5 \\
\hline 217941732 & 1 & 2.49412 & 0.015 & 0.001 & 0.001 & 9.26 & 86.53 & 0.49 & 0.26 & 0.17 & 49.80 & 1.25 & 0.14 & 0.20 & 0.032 & 136.4 & 35.8 & 45.8 \\
\hline
\end{tabular}

Notes.

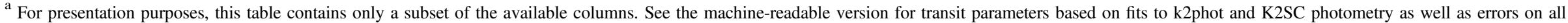
parameters.

${ }^{b}$ See Table 3 for errors on $P$ and estimates of the transit center.

${ }^{\mathrm{c}}$ This K2OI displays transit timing variations, but our fits assumed a linear ephemeris.

(This table is available in its entirety in machine-readable form.) 

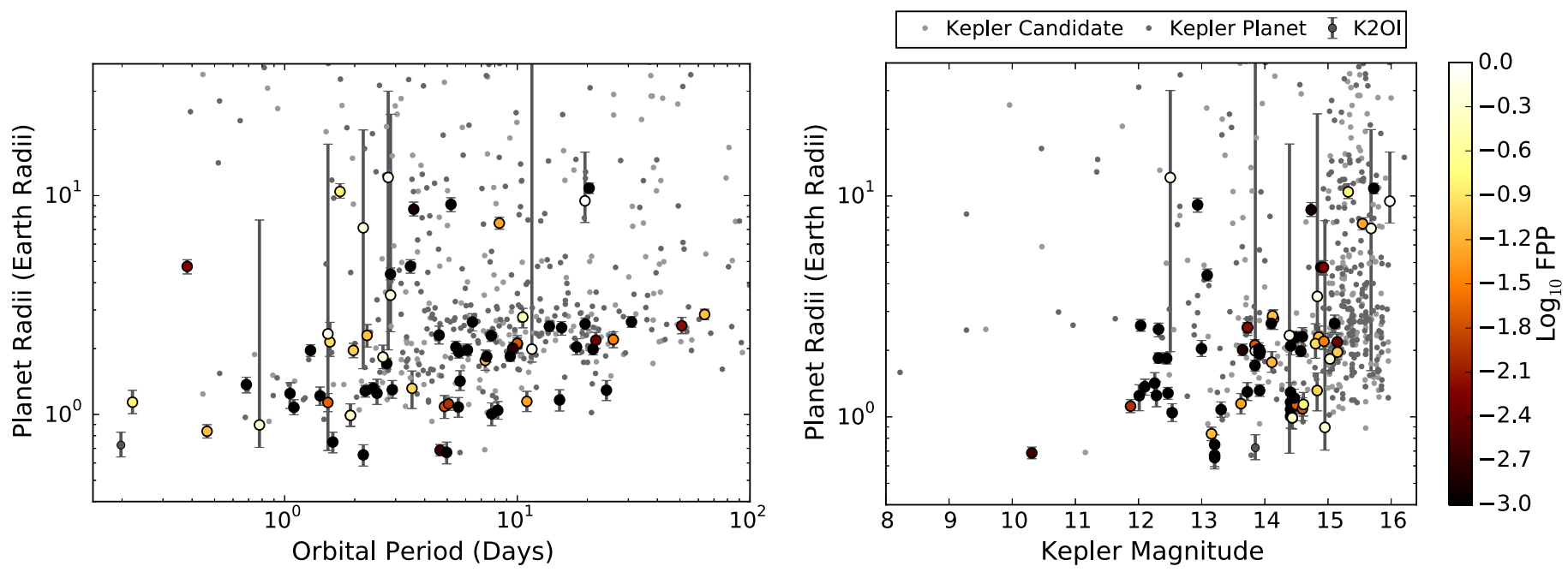

Figure 2. Revised radii vs. orbital period (left) or $K p$ (right) for all K2OIs observed in this work that were not classified as false positives. The colors indicate the average of the two FPP estimates for each K2OI as shown in the legend. For reference, the smaller circles mark planet candidates (light gray) and validated/confirmed planets (dark gray) detected during the prime Kepler mission. We obtained the properties of Kepler planets from the NASA Exoplanet Archive (Akeson et al. 2013).

candidates have radii $\lesssim 3 R_{\oplus}$, but both the Kepler and $\mathrm{K} 2$ radius distributions have tails extending to larger planet radii. As expected, Figure 2 also reveals that K2OIs with high FPPs tend to be larger than those with lower FPPs. Martinez et al. (2017) noted a similar size difference between the radii of their planet candidates and validated planets (see their Figure 9) and remarked that the radius estimates for candidates tend to be more uncertain.

The primary difference between our K2 planet candidates and the Kepler sample is that the $\mathrm{K} 2$ sample is biased toward brighter host stars. Considering only stars hosting planet candidates or validated planets, the median $K p$ of our cool dwarf sample is 14.1. As shown in the right panel of Figure 2, $77 \%$ of Kepler planets and planet candidates orbiting stars cooler than $4800 \mathrm{~K}$ have fainter host stars. The Kepler cool dwarf sample has a median host star brightness of $K p=15.2$, fainter than $93 \%$ of our K2 host stars. Interestingly, we note that our false positive sample is biased toward fainter host stars relative to the overall sample: the median $K p$ of our false positive sample is 14.8. A possible explanation for this trend is that the reduced photon counts for these faint stars cause the signal-to-noise ratio of the putative transit events to be below the threshold required to distinguish between bona fide transits and grazing eclipsing binaries.

In both the Kepler and K2 samples, we note a deficit of large planets at short orbital periods. The sole K2 planet with a period shorter than one day and a plotted radius larger than $1.5 R_{\oplus}$ is EPIC 201637175.01 (K2-22b), which is reported to be in the process of evaporating (Sanchis-Ojeda et al. 2015). Although our best-fit transit model uses a radius of $4.75_{-0.36}^{+0.35} R_{\oplus}$, Sanchis-Ojeda et al. (2015) argued that the planet itself is likely significantly smaller and surrounded by large dust clouds. In that interpretation, the deep transits are caused by the clouds of debris and do not reflect the underlying radius of the planet (Sanchis-Ojeda et al. 2015).

In general, the relative lack of larger short-period planets is consistent with results from the Kepler mission: Sanchis-Ojeda et al. (2014) observed that all ultra-short-period planets orbiting G, $\mathrm{K}$, or M dwarfs have radii $\lesssim 2 R_{\oplus}$. The lack of large planets on ultra-short orbital periods may indicate that the envelopes of highly irradiated planets are highly vulnerable to photoevaporation (e.g., Watson et al. 1981; Lammer et al. 2003; Baraffe et al. 2004; Murray-Clay et al. 2009; Valencia et al. 2010; Sanz-Forcada et al. 2011; Lopez et al. 2012; Kurokawa \& Kaltenegger 2013; Lopez \& Fortney 2013; Owen \& Wu 2013). At longer orbital periods, our sample includes planet candidates and validated planets with estimated radii between $0.7 R_{\oplus}$ and $12.1 R_{\oplus}$.

\subsection{Biases in the Planet Sample}

Adopting the same planet size categories as Fressin et al. (2013), our sample contains 21 Earths $\left(<1.25 R_{\oplus} ; 8\right.$ validated), 18 super-Earths $\left(1.25-2 R_{\oplus} ; 13\right.$ validated), 21 small Neptunes (2-4 $R_{\oplus} ; \quad 13$ validated), three large Neptunes (4-6 $R_{\oplus}$; two validated), and eight giant planets ( $>6 R_{\oplus}$; two validated). The size distribution depicted in Figure 3 is not representative of the larger population of planets orbiting low-mass stars. Rather, our sample, like most K2 planet catalogs, is shaped by strong selection biases due to the increased detectability of planets on short-period orbits compared to planets with longer periods and our interest in identifying compelling small planets orbiting bright stars for future follow-up observations.

Looking at Figure 4, the smallest planets are predominantly detected around the coolest target stars (median $T_{\text {eff }}=3595 \mathrm{~K}$ for $R_{p}<1.25 R_{\oplus}$ compared to $T_{\text {eff }}=3842 \mathrm{~K}$ for the full sample of planet candidates and validated planets), but the observed correlation of planet radius and stellar effective temperature is likely a selection effect due to the $1 /\left(R_{*}\right)^{2}$ scaling of transit depth with stellar radius rather than a reflection of the true underlying occurrence rate of small planets. We further investigate the role of selection biases in Figure 5 by comparing the host star magnitudes and radii for different sizes of planets.

As would be expected if the minimum planet radius were a function of search sensitivity, we find that our smallest planet candidates and validated planets are preferentially associated with the brightest and smallest host stars. We also note that the Neptunes and giant planets in the sample fall along the lower right edge of the distribution, indicating that they tend to orbit stars that are fainter and/or larger than the majority of the stars in our target sample. We interpret both of these results as evidence that our planet sample contains significant selection effects and 


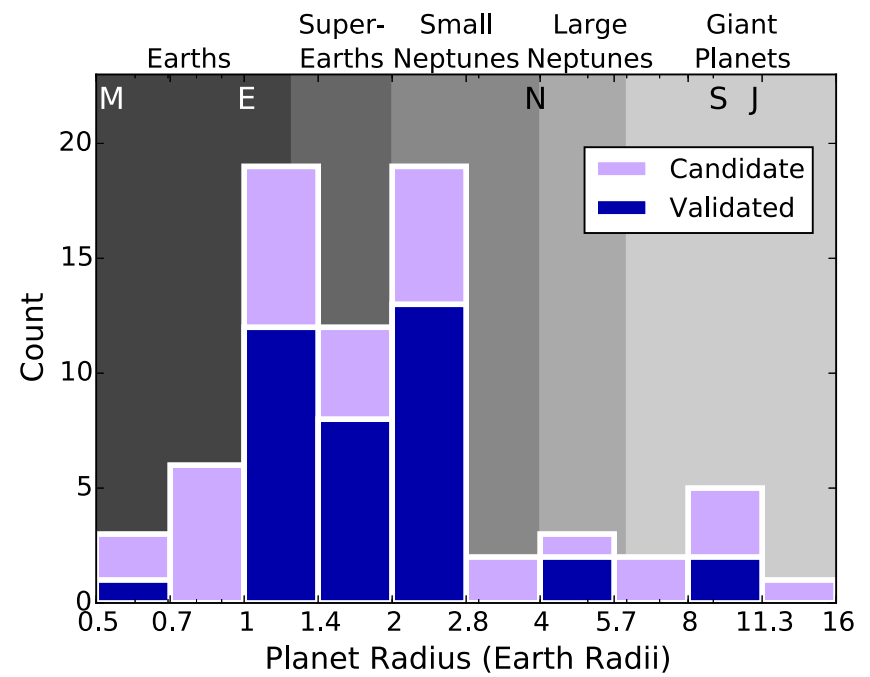

Figure 3. Stacked histogram displaying the size distribution for the population of K2 planet candidates (lilac) and validated planets (navy) characterized in this paper. The background shading denotes the planet size ranges defined by Fressin et al. (2013) and adopted in this paper. Letters mark the radii of Mars, Earth, Neptune, Saturn, and Jupiter.

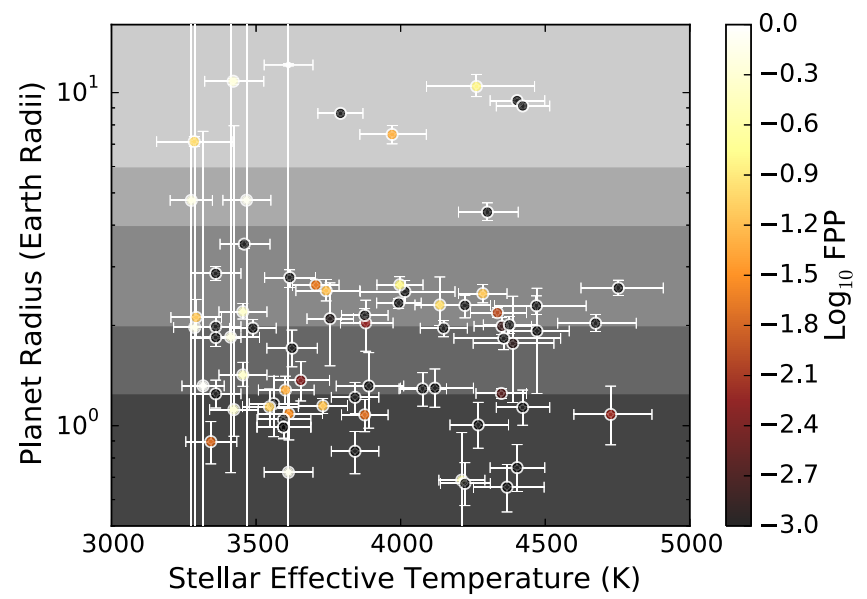

Figure 4. Revised radii of $\mathrm{K} 2$ planet candidates and validated planets vs. the effective temperature of their host stars. As in Figure 2, the planets are colorcoded according to their FPPs. The shaded regions are the same as in Figure 3 and denote Earths (darkest region; at bottom), super-Earths (second from bottom), small Neptunes (middle), large Neptunes (second from top), and giant planets (lightest region; at top).

therefore cannot be used to estimate planet occurrence rates unless the detection biases are considered as part of the analysis.

\subsection{Planet Radii versus Insolation Flux: Photoevaporation and Potentially Habitable Systems}

In Figure 6 we plot the insolation flux received by the planet candidates and validated planets in our sample as a function of the effective temperatures of their host stars. We calculate the insolation flux from the stellar luminosities and the orbital semimajor axes, which we derive from the orbital periods and the stellar masses.

Due to the relatively short, 80 day durations of K2 campaigns and the bias of the transit method toward shortperiod planets, the majority of the planets have very short orbital periods and are therefore highly irradiated. The most heavily irradiated planets in our sample receive over

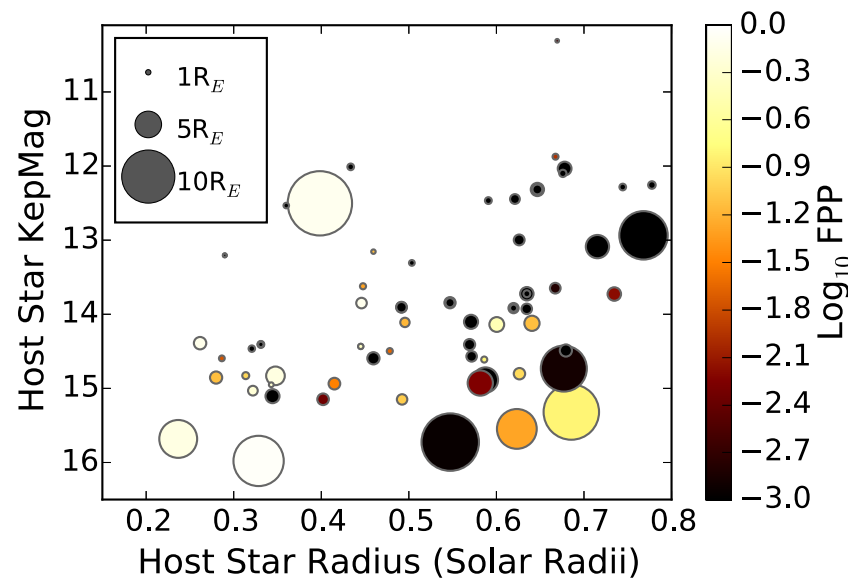

Figure 5. Host star magnitude in the Kepler bandpass vs. estimated stellar radius for K2 targets in our low-mass candidate host star sample. Larger circles indicate larger planets and the planets are colored based on their FPPs using the same scaling as in Figure 2.

1000 times the flux received by the Earth $\left(F_{\oplus}\right)$ and $21 \%$ of the planets receive at least $100 F_{\oplus}$.

Although our ability to discern relationships between planet radii and insolation flux environment is complicated by selection effects, Figure 6 reveals that the population of highly irradiated planets is dominated by smaller planets. As previously discussed, the shortage of highly irradiated intermediate-sized planets may be due to photoevaporation. Of the seven validated planets and planet candidates receiving at least $200 F_{\oplus}$, the only object with a radius between $2 R_{\oplus}$ and $10 R_{\oplus}$ is the evaporating planet EPIC 201637175.01 (K2-22b Sanchis-Ojeda et al. 2015).

At the opposite extreme of the insolation flux distribution, our cool dwarf sample contains 21 planets or planet candidates receiving $<10 F_{\oplus}$. In order to assess whether any of these planets might be habitable, we used the polynomial relations from Kopparapu et al. (2013) to determine the insolation flux boundaries corresponding to conservative habitable zone (HZ) limits of the moist greenhouse inner edge and the maximum greenhouse outer edge and to more optimistic limits of recentVenus inner limit and early-Mars outer limit. A more sophisticated choice would have been to use the planet-mass-dependent relations from Kopparapu et al. (2014), but we do not know the masses of these planets.

Given the temperature distribution of the host stars in our cool dwarf sample, the median $\mathrm{HZ}$ boundaries are $0.26-0.89 F_{\oplus}$ $(0.22-0.40 \mathrm{au})$ for the conservative case and $0.23-1.55 F_{\oplus}$ (0.16-0.42 au) for the more optimistic case. As shown in Figure 6, four of the planets and planet candidates in our sample fall within the optimistic HZ limits (EPIC 206209135.04, 211799258.01, 211988320.01, and 212690867.01). We discuss each of these K2OIs individually in Section 7.

\subsection{Comparison of K2 Photometric Pipelines}

In order to investigate the influence of systematic effects in the reduced $\mathrm{K} 2$ photometry on the derived planet properties, we repeated the transit fits using photometry processed by the K2 Systematics Correction Pipeline ${ }^{26,27}$ (Aigrain et al. 2015, 2016) and the k2phot pipeline (Petigura et al. 2013). We note that

\footnotetext{
26 https://archive.stsci.edu/prepds/k2sc/; https://doi.org/10.17909/T9K591

27 https://github.com/OxES/k2sc
} 


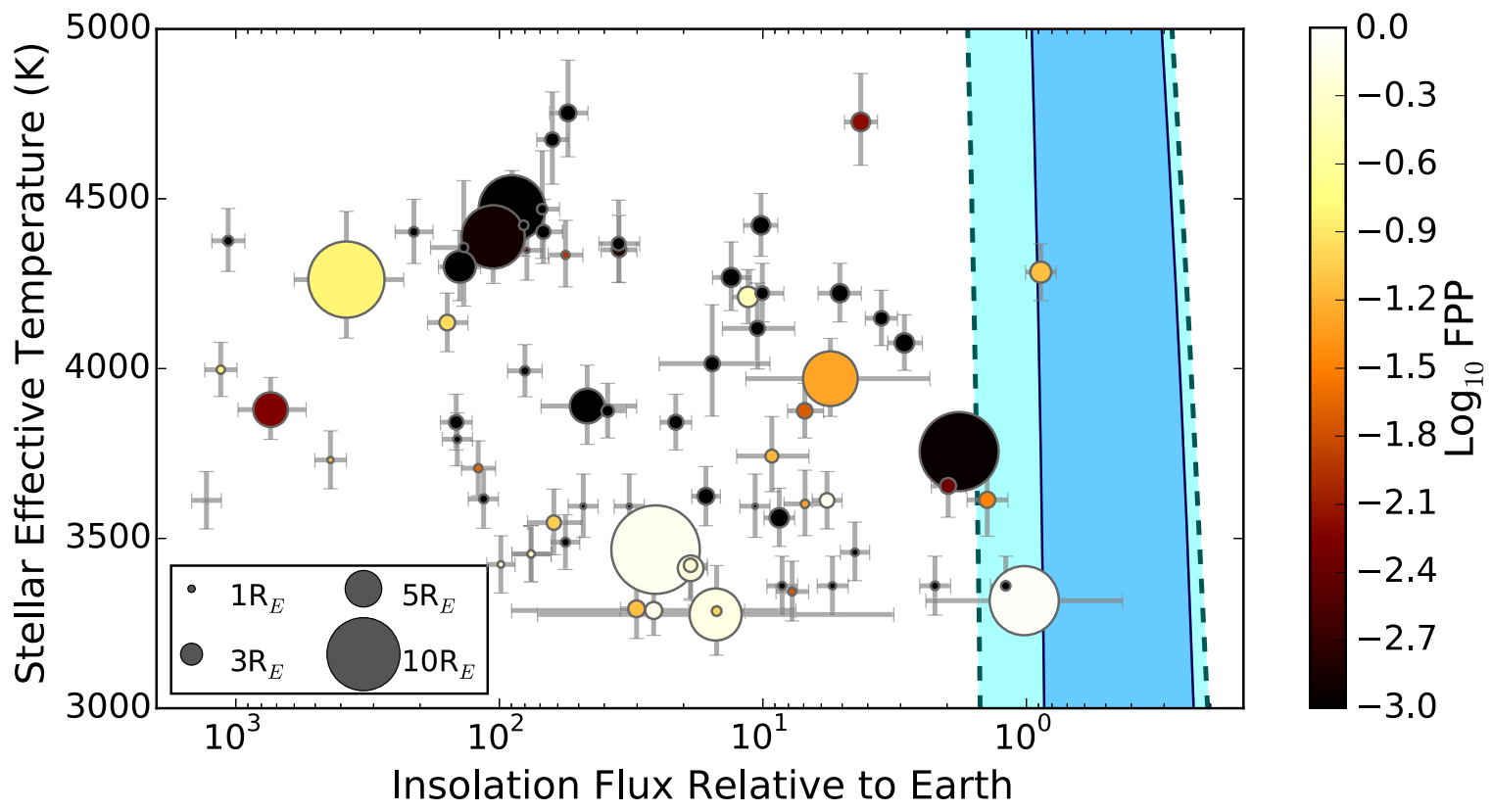

Figure 6. Revised stellar effective temperatures vs. the insolation flux received by associated planets. As indicated by the legend and colorbar, the data points are scaled by planet radius and colored based on FPP. The cyan and blue regions indicate optimistic (early-Venus/recent-Mars) and pessimistic (moist greenhouse/ maximum greenhouse) habitable zone boundaries based as calculated by Kopparapu et al. (2013). The planets plotted within the habitable zone are: EPIC 206209135.04 (small black circle; $1.3 \pm 0.1 R_{\oplus}, F_{p}=1.2 \pm 0.2 F_{\oplus}$, FPP $=1 \times 10^{-5}-8 \times 10^{-5}$ ), EPIC 211799258.01 (large white circle; $R_{p}=9.5_{-1.9}^{+6.3} R_{\oplus}$, $F_{p}=1.0_{-0.6}^{+1.4} F_{\oplus}, \quad$ FPP $=90 \%-94 \%$ ), EPIC $211988320.01 \quad$ (medium yellow-orange circle; $R_{p}=2.9 \pm 0.2 \quad R_{\oplus}, \quad F_{p}=0.9 \pm 0.1 F_{\oplus}, \quad$ FPP $=7 \%$ ), and EPIC 212690867.01 (medium orange circle; $R_{p}=2.2 \pm 0.2 R_{\oplus}, F_{p}=1.4_{-0.2}^{+0.3} F_{\oplus}$, FPP $=3 \%-4 \%$ ).
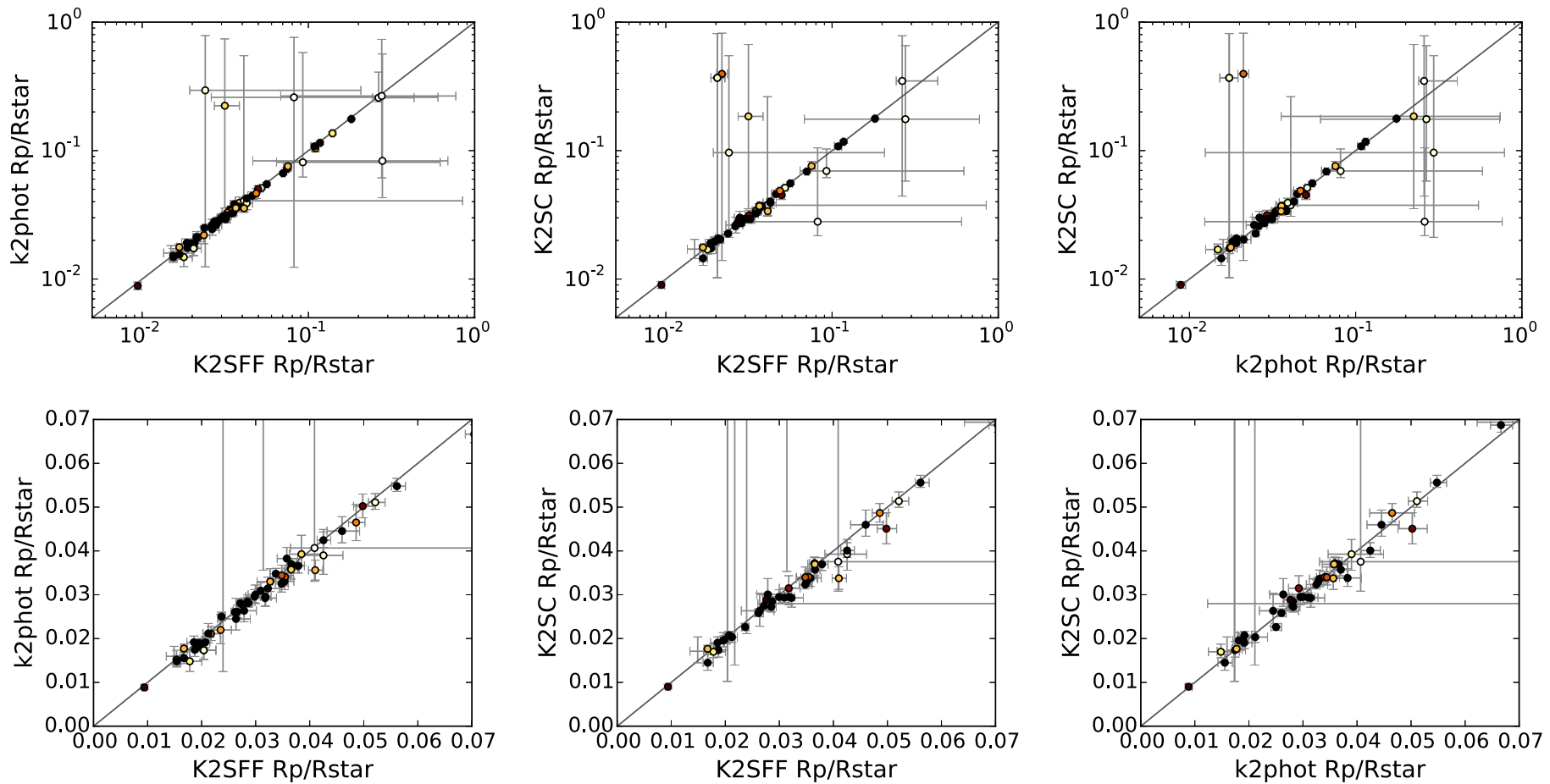

Figure 7. Comparison of planet/star radius ratios found by fitting photometry from different pipelines. The symbols are colored based on FPP using the same color scaling as in Figure 11; K2OIs with lower FPPs have darker colors. The top panels display all K2OIs while the bottom panels zoom in to highlight the K2OIs with small $R_{p} / R_{\star}$ ratios. Only planet candidates and validated planets are shown. Left: k2phot fits vs. K2SFF fits. Middle: K2SC fits vs. K2SFF fits. Right: K2SC fits vs. k2phot fits.

lightcurves produced using the k2varcat (Armstrong et al. 2014, 2015, 2016) and EVEREST (Luger et al. 2016) pipelines are also available on the MAST, but we did not perform fits using those lightcurves.
Figure 7 compares the resulting planet/star radius ratios found by fitting photometry from the $\mathrm{K} 2 \mathrm{SFF}, \mathrm{K} 2 \mathrm{SC}$, and k2phot pipelines. For K2OIs with small $R_{\mathrm{p}} / R_{\star}$, we find generally consistent planet properties regardless of our choice of photometric 
pipeline, but the estimates for K2OIs with $R_{p} / R_{\star}>0.05$ can be quite discrepant. In general, the K2OIs with the largest radius disagreement are false positives for which the transit model provides a poor fit to the data. In contrast, nearly all of the K2OIs with $R_{p} / R_{\star}>0.05$ appear well-fit by a transit model and many were classified as confirmed planets in Section 5 based on their K2SFF lightcurves.

Specifically, the median absolute difference in $R_{p} / R_{\star, \mathrm{KSFF}}$ and $R_{p} / R_{\star, \mathrm{k} 2 \mathrm{phot}}$ is $\Delta R_{p} / R_{\star, \mathrm{K} 2 \mathrm{SFF}-\mathrm{k} 2 \mathrm{phot}}=0.001$ for validated planets, $\Delta R_{p} / R_{\star, \mathrm{K} 2 \mathrm{SFF}-\mathrm{k} 2 \mathrm{phot}}=0.002$ for planet candidates, and $\Delta R_{p} / R_{\star, \mathrm{K} 2 \mathrm{SFF}-\mathrm{k} 2 \mathrm{phot}}=0.05$ for false positives. Comparing the $\mathrm{KSFF}$ and K2SC fits, we find similar median differences of 0.001 for validated planets and 0.003 for planet candidates, but a higher median difference of 0.12 for false positives. These values are comparable to the median differences of $0.001,0.002$, and 0.06 found when comparing the $R_{\mathrm{p}} / R_{\star}$ fits from the k2phot and $\mathrm{K} 2 \mathrm{SC}$ pipelines for validated planets, planet candidates, and false positives, respectively.

Despite the overall agreement between the transit fits produced using distinct sets of photometry, there are a few K2OIs with large $R_{p} / R_{\star}$ differences across pipelines. For instance, we noticed that the K2SC pipeline appeared to remove the transits of EPIC 211799258.01. Furthermore, our k2phot fits for EPIC 211826814.01 and EPIC 212443973.01 and our K2SC fits for EPIC 206312951.01 and EPIC 211694226.01 failed to converge on the transit midpoint. In general, we found that comparing fits from different pipelines was a convenient way to bolster confidence in borderline detections and reject astrophysical false positives due to blended photometry.

\subsection{Updates to Transit Parameters}

As shown in Table 2, many of the K2OIs analyzed in this paper were previously announced in other publications. Consulting the NASA Exoplanet Archive (Akeson et al. 2013), we found that 27, 17, 5, and 3 of our K2OIs were included in the candidate catalogs published by Crossfield et al. (2016), Vanderburg et al. (2016b), Montet et al. (2015), and Adams et al. (2016), respectively. In addition, 18 K2OIs were included in the Barros et al. (2016) catalog, 15 appeared in the Pope et al. (2016) catalog, and one (EPIC 205924614.01 = K2-55b) was part of the Schmitt et al. (2016) catalog. While the first set of catalogs included both transit parameters and physical properties like planetary and stellar radii, the second set did not convert transit parameters to physical properties. We compare the available fits for all K2OIs in Figure 8.

As shown in the top left panel, our period and planet/star radius ratio estimates are generally in agreement with past results. The median differences $\left(R_{p} / R_{\star}\right)_{\text {revised }}-\left(R_{p} / R_{\star}\right)_{\text {literature }}$ between our revised planet/star radius ratios and previously published values are 0.0005 (Adams et al. 2016, three K2OIs), -0.001 (Barros et al. 2016, 18 K2OIs, standard deviation $\sigma=0.003$ ), 0.002 (Crossfield et al. 2016, $27 \mathrm{~K} 2 \mathrm{OIs}$, $\sigma=0.072$ ), 0.002 (Pope et al. 2016, $15 \mathrm{~K} 2 \mathrm{OIs}, \sigma=0.003$ ), -0.0007 (Montet et al. 2015, five K2OIs, $\sigma=0.005$ ), -0.001 (Schmitt et al. 2016, EPIC 205924614.01), and -0.0006 (Vanderburg et al. 2016b, $17 \mathrm{~K} 2 \mathrm{OIs}, \sigma=0.002$ ).

The right panels of Figure 8 reveal that our stellar radius estimates tend to be larger than the values used in previous studies. Specifically, we find median differences of $0.04 R_{\odot}$, $0.13 R_{\odot}, \quad-0.03 R_{\odot}$, and $0.01 R_{\odot}$ compared to the radius estimates from Adams et al. (2016, three K2OIs), Crossfield et al. (2016, $27 \mathrm{~K} 2 \mathrm{OIs}, \sigma=0.07$ ), Montet et al. (2015, five
K2OIs, $\sigma=0.09$ ), and Vanderburg et al. (2016a, 17 K2OIs, $\sigma=0.10$ ). Primarily due to the large difference between our stellar radius estimates, our planet radius estimates are typically $0.5 R_{\oplus}(35 \%)$ larger than those from Crossfield et al. (2016, $\left.\sigma=3 R_{\oplus}\right)$. We measure smaller differences of $-0.01 R_{\oplus}$ $(-1 \%),-0.20 R_{\oplus}(-9 \%)$, and $-0.13(-5 \%)$ between our values and those from Adams et al. (2016, three K2OIs), Montet et al. (2015, five K2OIs, $\sigma=1 R_{\oplus}$ ), and Vanderburg et al. (2016a, $17 \mathrm{~K} 2 \mathrm{OIs}, \sigma=0.5 R_{\oplus}$ ), respectively. In comparison, our typical errors on planet radii are 6\%-13\%.

The two K2OIs with large changes are EPIC 201617985.01 and EPIC 201637175.01 (K2-22b). Crossfield et al. (2016) reported $P=1.143201 \pm 0.000012$ days for EPIC 201637175.01 and $R_{p} / R_{\star}=0.41_{-0.34}^{+0.34}$ for EPIC 201617985.01 whereas we found $P=0.38$ days and $R_{p} / R_{\star}=0.033_{-0.024}^{+0.032}$. Our shorter orbital period for EPIC 201637175.01 is one third of the value reported by Crossfield et al. (2016) and matches earlier estimates by Sanchis-Ojeda et al. (2015), Vanderburg et al. (2016b), and Adams et al. (2016). Similarly, our revised planet/star radius estimate for EPIC 201617985.01 is in agreement with previous estimates by Montet et al. $\left(2015, R_{p} / R_{\star}=0.033\right)$ and Vanderburg et al. (2016a, $\left.R_{p} / R_{\star}=0.032\right)$.

We attribute the period error for EPIC 201637175.01 to the fact that the TERRA search did not consider threshold-crossing events with periods shorter than a day (Crossfield et al. 2016). For EPIC 201617985.01, the Crossfield et al. (2016) fit favored a large impact parameter $\left(b=1.38_{-0.31}^{+0.41}\right)$, which led to a broad posterior distribution of allowed planet radii. Excluding those two candidates does not alter the median differences between our revised values and those published by Crossfield et al. (2016), but considerably shrinks the standard deviations of the differences from 0.072 to 0.002 for the planet/star radius ratios and from $3 R_{\oplus}$ to $0.8 R_{\oplus}$ for the planet radii.

\section{Discussion}

Several of the planets in our sample are particularly interesting because they might be habitable, reside in systems with multiple transiting planets, or orbit particularly bright stars. For reference, we present schematics of all of the multiplanet systems and potentially habitable systems in Figure 9. We also discuss individual systems in the following sections.

For the purpose of planning potential follow-up observations, we first employed the planetary mass-radius relation presented in Weiss \& Marcy (2014) to assign masses to the K2OIs and computed the expected radial velocity (RV) signal due to each planet. We display the resulting estimates in Figure 10. We note that the full range of small planet compositions is not well captured by a one-to-one mass-radius relation (Wolfgang \& Lopez 2015; Chen \& Kipping 2017), but these coarse mass estimates are sufficient for assessing the feasibility of future RV investigations.

In general, we find that these planets would be challenging targets for current RV spectrographs due to the small expected signal (median value $=2.3 \mathrm{~m} \mathrm{~s}^{-1}$ ) and the faintness of their host stars at optical wavelengths. In addition, several of the easiest candidates in Figure 10 are unsuitable for RV observations: EPIC 210558622 was identified as an spectroscopic binary (Crossfield et al. 2016), EPIC 212679181 is accompanied by a fainter star 1."5 away, ${ }^{28}$ and EPIC 216892056.01 is almost certainly a false positive.

\footnotetext{
${ }^{28}$ https://exofop.ipac.caltech.edu/k2/edit_obsnotes.php?id=212679181
} 


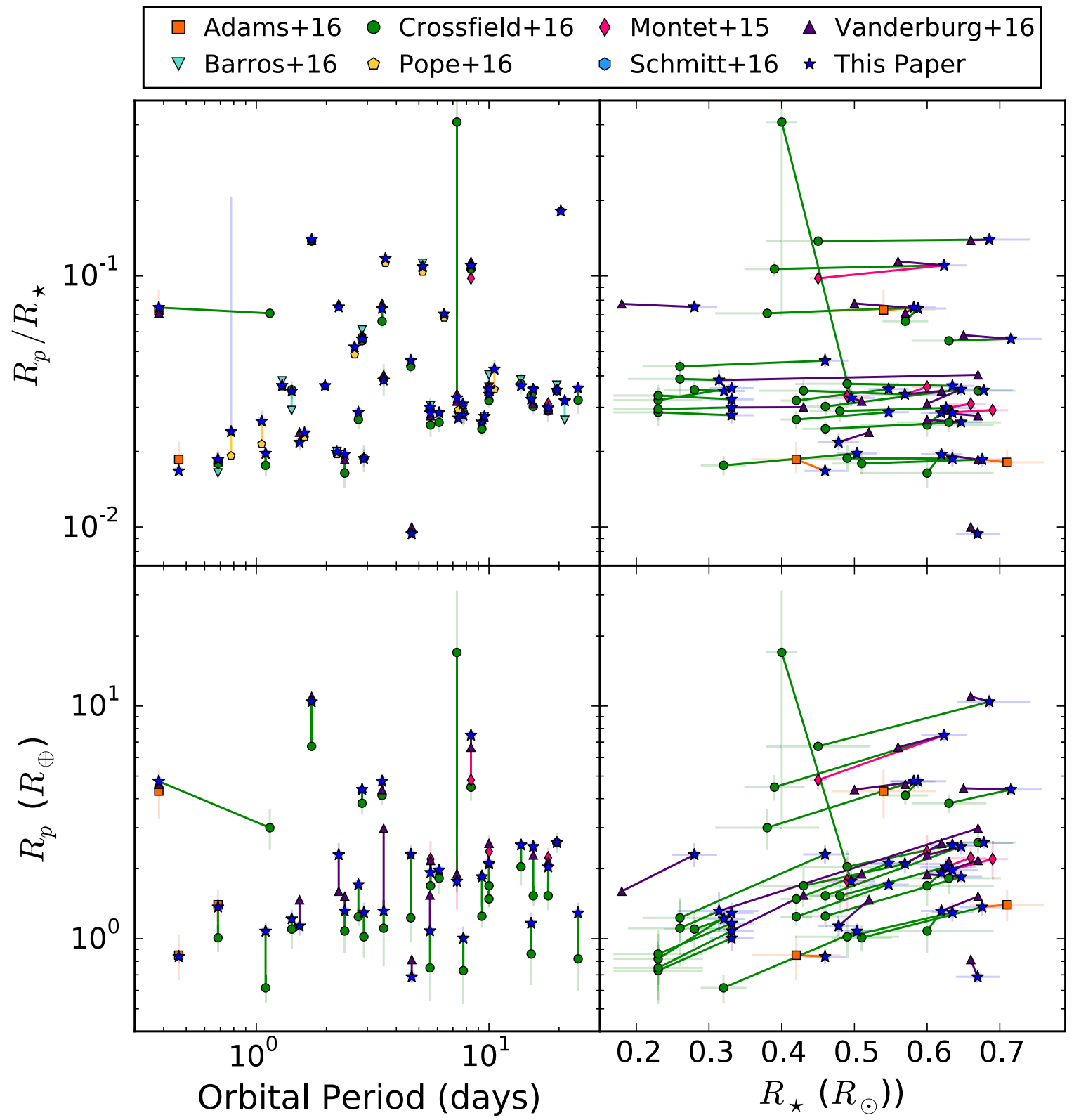

Figure 8. Comparison of our updated stellar and planetary properties (blue stars) to previously published values from Adams et al. (2016, orange squares), Barros et al. (2016, teal downward triangles), Crossfield et al. (2016, green circles), Pope et al. (2016, yellow pentagons), Montet et al. (2015, pink diamonds), Schmitt et al. (2016, light blue hexagons), and Vanderburg et al. (2016a, purple upward triangles). Where available, we display the errors using faded lines in the color assigned to each paper. The darker lines connect our revised values to the previously published estimates. Top left: planet/star radius ratio vs. orbital period. Top right: planet/star radius ratio vs. stellar radius. Bottom left: planet radius vs. orbital period. Bottom right: planet radius vs. stellar radius. Note that not all earlier papers contained estimates of physical planetary and stellar radii.

In contrast, the small Neptune EPIC 205924614.01 (K2-55b) is an ideal follow-up target and has already been observed by both Keck/HIRES and Spitzer. The validated small planets EPIC 210707130.01 and 212460519.01 are also attractive RV targets. We discuss K2-55b in detail in a separate publication (C. D. Dressing et al. 2017, in preparation) and consider the two smaller planets in Section 7.3.

Although our targets are generally poorly suited for RV mass measurements, they are more compelling candidates for transmission spectroscopy. The combination of the small sizes and red colors of their host stars produces larger transmission signals than would be expected for similar planets orbiting Sunlike stars with similar brightnesses in the Kepler bandpass.
While our targets are moderately faint at visible wavelengths, they are modestly bright in the NIR. As shown in Figure 11, several planets should yield detectable transmission signals if our assumptions regarding planet masses and atmospheric compositions are correct.

We approximated the transmission signals shown in Figure 11 by assuming that the planetary atmospheres extend for five scale heights. When calculating the latter, we adopted the same planet masses as for the RV predictions and determined planetary equilibrium temperatures using a fixed planetary albedo of 0.3 . We also assumed that each planet possesses a Jupiter-like atmosphere with a mean molecular weight of $2.2 m_{H}$; planets with water-dominated atmospheres would generate transmission 

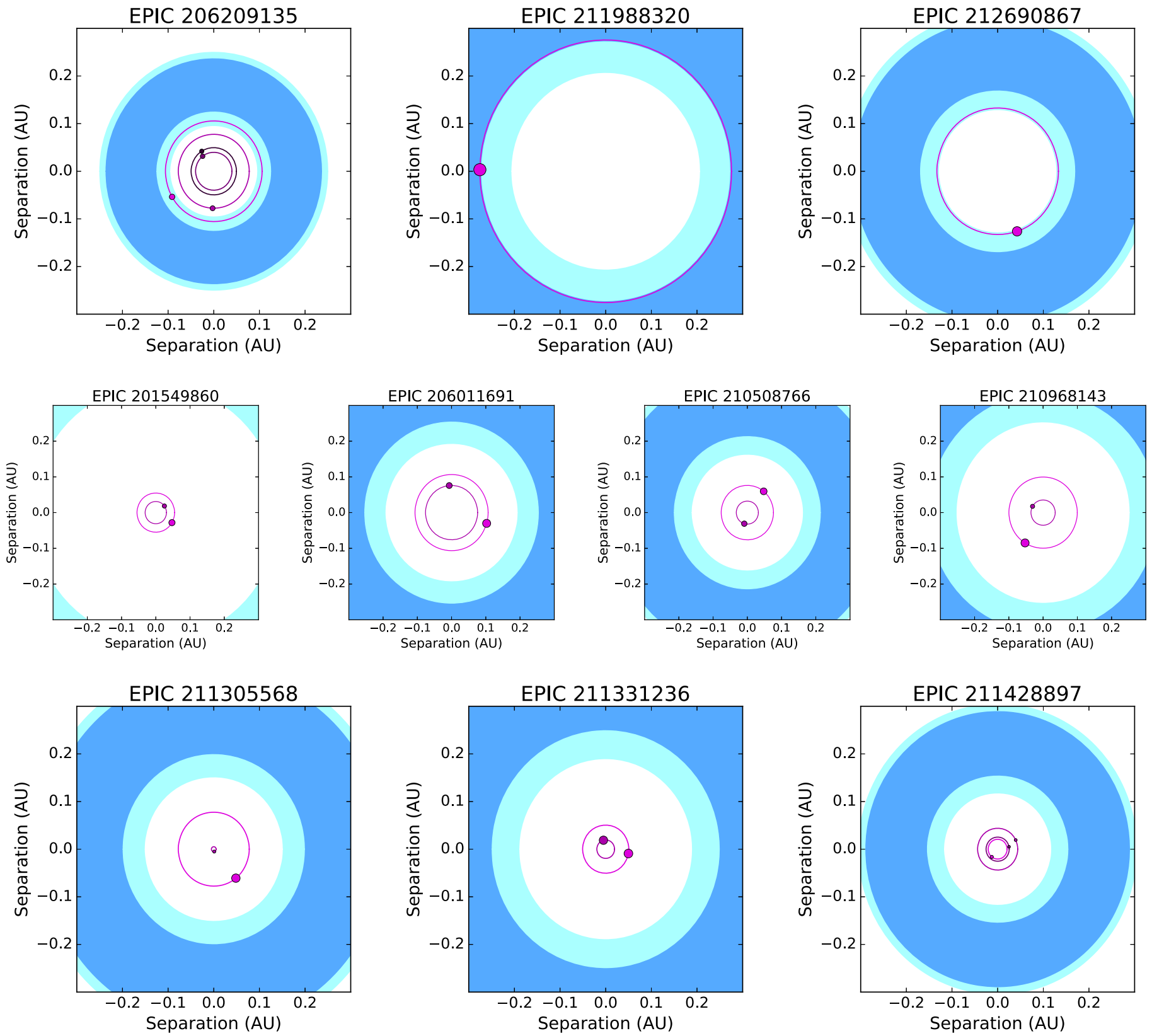

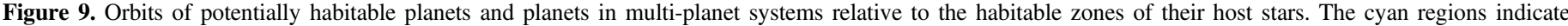

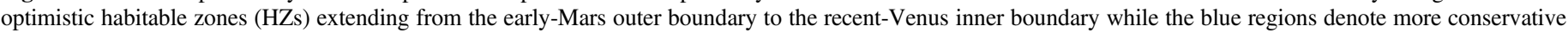

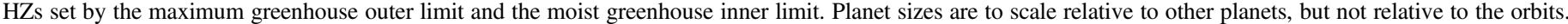

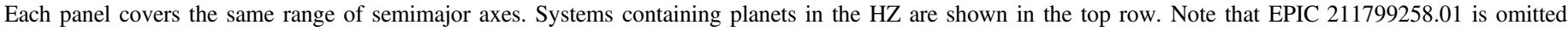
because it is almost certainly a false positive (see Section 7.1.2).

signals an order of magnitude smaller. While exciting from a habitability standpoint, the smallest planets in our sample may be bare rocky cores without substantial atmospheres and would therefore be poor targets for transmission spectroscopy.

Consulting Figure 11, the most intriguing targets for atmospheric characterization are EPIC 201205469.01 (K2-43b, Crossfield et al. 2016), 201345483.01 (K2-45b, Crossfield et al. 2016), 201635569.01 (K2-14b, Montet et al. 2015; Crossfield et al. 2016), 205924614.01 (K2-55b, Crossfield et al. 2016), 206318379.01 (K2-28b, Hirano et al. 2016; G. Chen et al. 2017, in preparation), 211509553.01, 211818569.01 (K2-121b), and 212554013.01
(K2-127b). These planets span a broad range in both size and equilibrium temperature: EPIC 211509553.01 is a cool giant planet $\left(R_{p}=10.8 \pm 0.6 R_{\oplus}, T_{\mathrm{eq}}=355 \mathrm{~K}\right)$; EPIC 201345483.01 is a hot giant planet $\left(R_{p}=10.4_{-0.7}^{+0.9} R_{\oplus}, T_{\mathrm{eq}}=988 \mathrm{~K}\right)$; EPIC 201635569.01, EPIC 211818569.01, and EPIC 212554013.01 are warm giant planets $\left(R_{p}=7.5 \pm 0.5 R_{\oplus}, T_{\mathrm{eq}}=527 \mathrm{~K} ; R_{p}=\right.$ $9.2 \pm 0.7 R_{\oplus}, T_{\mathrm{eq}}=756 \mathrm{~K}$; and $R_{p}=8.7_{-0.6}^{+0.7} R_{\oplus}, T_{\mathrm{eq}}=789 \mathrm{~K}$, respectively); EPIC 201205469.01 and EPIC 205924614.01 are warm large Neptunes $\left(R_{p}=4.8 \pm 0.3 R_{\oplus}, T_{\text {eq }}=676 \mathrm{~K}\right.$ and $R_{p}=4.4 \pm 0.3 R_{\oplus}, T_{\mathrm{eq}}=861 \mathrm{~K}$, respectively); and EPIC 20631 8379.01 is a warm small Neptune $\left(R_{p}=2.3 \pm 0.3 R_{\oplus}, T_{\mathrm{eq}}=\right.$ 

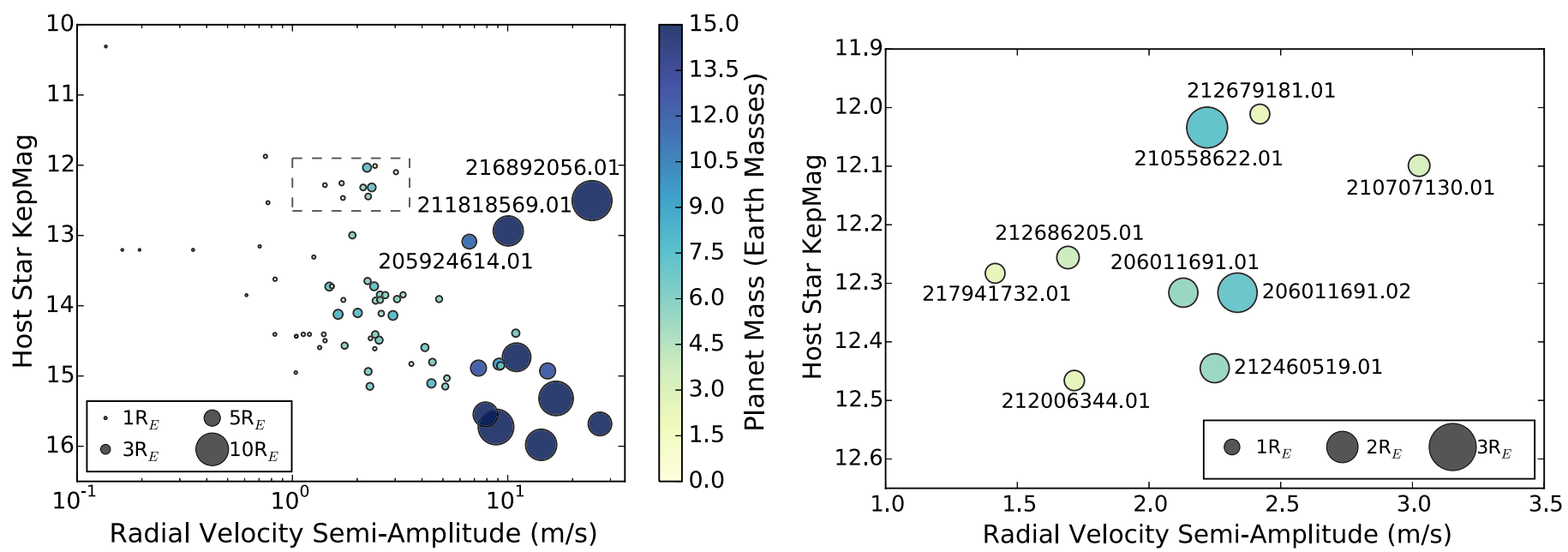

Figure 10. Host star Kepler magnitude vs. expected radial velocity (RV) semi-amplitude. The sizes of data points are scaled so that larger planets have bigger data points and the colors indicate the assumed planet masses. Left: estimated RV semi-amplitudes for all of the planets in our sample. Right: estimated RV semiamplitudes of the small planets most amenable to RV observations. This panel shows a zoom-in of the boxed region in the left panel. We label key planets for ease of reference and discuss the most promising systems individually later in this section.

$547 \mathrm{~K})$. Of these planets, EPIC 205924614.01, 20618379.01, and 211818569.01 have the brightest host stars and are therefore the most favorable targets.

\subsection{Systems with Planets in or Near the $H Z$}

\subsubsection{EPIC 206209135 (K2-72): Validated Four-planet System with an Earth-sized Planet in the $\mathrm{HZ}$}

EPIC 206209135 is an M2 dwarf ( $K p=14.407$, $K s=10.962)$ orbited by four transiting planets with periods of 5.577, 7.760, 15.189, and 24.167 days. All four planets were previously validated by Crossfield et al. (2016) based on careful scrutiny of the $\mathrm{K} 2$ photometry, follow-up imaging with Keck/NIRC2, and low FPPs as computed with VESPA (Morton 2015b). In their analysis, Crossfield et al. (2016) assumed a stellar radius of $0.232 \pm 0.056 R_{\odot}$, which is $30 \%$ smaller than our revised value of $0.33 \pm 0.03 R_{\odot}$ (D17). Despite the significant change in the assumed stellar parameters, our independent VESPA analysis also returned FPPs low enough to validate all four planets.

The outermost planet (EPIC 206209135.04) has a radius of $1.29_{-0.13}^{+0.14} R_{\oplus}$, a semimajor axis of $0.106_{-0.013}^{+0.009}$ au and an insolation flux of $1.2 \pm 0.2 F_{\oplus}$, placing it within the $0.22-1.52 F_{\oplus}(0.09-0.25 \mathrm{au})$ boundaries of the optimistic Venus/Mars HZ but outside the $0.28-0.91 F_{\oplus}(0.13-0.24 \mathrm{au})$ limits of the conservative moist greenhouse/maximum greenhouse HZ. The second outermost planet (EPIC 206209135.02) is slightly smaller $\left(R_{p}=1.16 \pm 0.13 R_{\oplus}\right)$ and lies inside the inner edge of the optimistic $\mathrm{HZ} \quad\left(F_{p}=2.2 \pm 0.3 F_{\oplus}\right.$, $a=0.078_{-0.010}^{+0.007}$ ). The two innermost planets (EPIC 20 6209135.01 and EPIC 206209135.03) have radii of $1.08 \pm 0.11 R_{\oplus}$ and $1.01 \pm 0.12 R_{\oplus}$ and receive $8.5_{-1.1}^{+1.2} F_{\oplus}$ and $5.4_{-0.7}^{+0.8} F_{\oplus}$, respectively. We note that our radius estimates for all four planets are consistent with the revised values found by Martinez et al. (2017), who used NTT/SOFI spectroscopy to characterize the host star and then scaled the $R_{p} / R_{\star}$ values from Crossfield et al. (2016) accordingly.

The orbital periods of the four planets are in near-resonant configurations, which suggests that the system may have experienced disk-driven migration. Specifically, planets .01 and .03 (5.577 days and 7.760 days) are near the second-order 7:5 mean motion resonance and planets .03 and .02 (7.760 days and 15.189 days) are near the first-order 2:1 mean motion resonance. Planets .01 and .04 (5.577 days and 24.167 days) have an orbital period ratio of roughly 3:13.

Assuming that all four planets have rocky compositions and masses of 1.1-2.6 $M_{\oplus}$ as suggested by the Weiss \& Marcy (2014) mass-radius relation, the expected radial velocity perturbations from each planet individually would have semiamplitudes of $0.8-1.4 \mathrm{~m} \mathrm{~s}^{-1}$. Given that the host star is relatively faint $(K p=14.407, K s=10.962)$ the system would be a challenging target for RV surveys. Fortunately, the nearresonant architecture of the system may enable planet mass measurements via transit timing variations (TTVs) rather than RVs. These TTV-based masses will be useful for interpreting the results of subsequent atmospheric characterization studies.

\subsubsection{EPIC 211799258: New Candidate Cool Jupiter}

This previously unknown planet candidate has a high FPP of $90 \%-94 \%$, suggesting that the system is most likely a false positive. Our Gemini-N/NIRI imaging of the system was sensitive to companions 6.6 (7.6) magnitudes fainter than EPIC 211799258 at $0.5\left(1{ }^{\prime \prime} 0\right)$ and did not reveal any companions, but our VESPA analysis suggested that the most likely false positive configuration for this system was an eclipsing binary that we would not expect to resolve in our $\mathrm{AO}$ imaging. If real, the planet candidate is a $9.5_{-1.9}^{+6.3} R_{\oplus}$ planet with an orbital period of 19.5 days. The host star is faint $(K p=15.979, K s=12.185)$, but the planet should generate a large RV signal. Given the paucity of cool Jupiters known to transit low-mass stars, this system may warrant further study despite the low likelihood that the planetary interpretation is correct. If this transit-like event is indeed caused by eclipses involving the target star, high-resolution spectra acquired at opposite quadratures should reveal significant RV variation. In contrast, the absence of RV variation would rule out the foreground eclipsing binary scenario and possibly allow us to validate EPIC 211799258.01 as a genuine planet. 

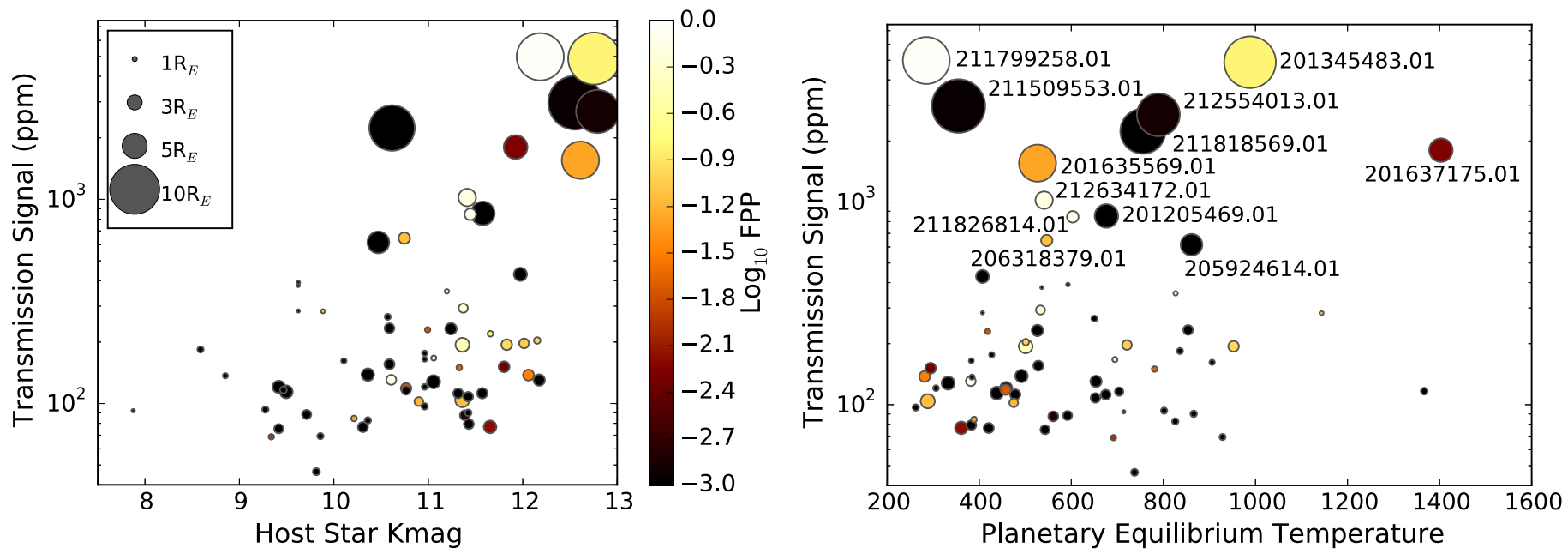

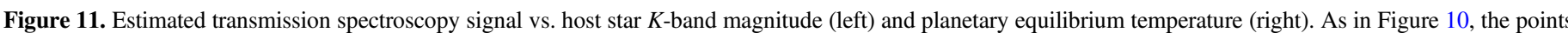

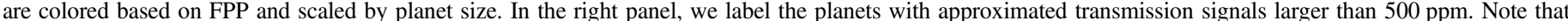

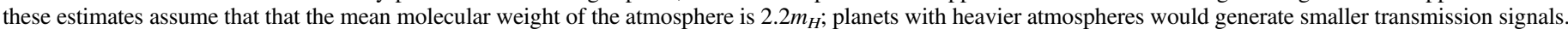

\subsubsection{EPIC 211988320: New Candidate Small Neptune in the HZ}

EPIC $211988320.01\left(2.86_{-0.15}^{+0.16} R_{\oplus}\right)$ was originally identified as a transit-like event with an orbital period of 15 days, but our subsequent inspection of the light curve revealed that the orbital period is actually 63.8 days. The longer orbital period places EPIC 211988320.01 just within the boundaries of the conservative moist greenhouse/maximum greenhouse $\mathrm{HZ}$ of its M0V host star. Although this planet is likely too large to be rocky, its host star is bright enough $(K p=14.122, K s=11.36)$ to enable future atmospheric characterization studies. Our estimated FPP for the candidate is $7 \%$, but our analysis did not include AO or speckle imaging. While the system is too faint for radial velocity $(K p=14.122, K s=11.360)$, acquiring AO or speckle images would be straightforward. Due to the long orbital period of EPIC 211983320.01, only two transits are visible in the $\mathrm{K} 2$ data. We therefore recommend acquiring additional transit observations to refine the orbital ephemeris prior to embarking upon an intensive atmospheric characterization campaign.

\subsubsection{EPIC 212690867: New Candidate Small Neptune in the HZ}

This small planet $\left(2.20_{-0.18}^{+0.19} R_{\oplus}\right)$ has an orbital period of 25.9 days around a $3614_{-107}^{+118} \mathrm{~K}$ host star. The planet receives an insolation flux of $1.4_{-0.2}^{+0.3} F_{\oplus}$ and would be an intriguing target for atmospheric characterization if its host star were slightly brighter $(K p=14.936, K s=12.061)$. Our VESPA analysis returned an FPP too large for validation (FPP $=3 \%-4 \%$ ), but the planet could likely be validated if we acquired an $\mathrm{AO}$ or speckle image to rule out the remaining false positive scenarios.

\subsubsection{EPIC 212398486 (K2-125): Newly Validated Mini-Neptune Near the $H Z$}

The EPIC 212398486 system consists of a mini-Neptune orbiting an M2 dwarf on a 21.8 day orbit. The $3654_{-92}^{+100} \mathrm{~K}$, $0.40 \pm 0.03 R_{\odot}$ host star has optimistic HZ boundaries of $0.2-1.5 F_{\oplus}$, just cooler than the $2.0 \pm 0.3 F_{\oplus}$ insolation flux received by the planet. At $R_{p}=2.19_{-0.18}^{+0.19} R_{\oplus}$, the planet is very unlikely to be rocky, but it is still an attractive target for followup atmospheric characterization studies. The majority of well- characterized mini-Neptunes are hot; the EPIC 212398486 system provides a complementary example of a cooler small planet. The system is fainter than most of our targets $(K p=15.147, \quad K s=11.802)$, but follow-up atmospheric studies should be feasible. Our VESPA analysis of the $\mathrm{K} 2 \mathrm{SFF}$ photometry yielded an FPP $=0.2 \%-0.7 \%$, allowing us to validate this previously unknown planet.

\subsection{Systems with Multiple Transiting Planets}

\subsubsection{EPIC 201549860 (K2-35): Validated Two-planet System}

The EPIC 201549860 (K2-35) system was validated by Sinukoff et al. (2016) as a $4680 \pm 60 \mathrm{~K}, 0.72 \pm 0.04$ star hosting a $1.40 \pm 0.17 R_{\oplus}$ inner planet with a period of 2.4 days and $2.09_{-0.31}^{+0.43} R_{\oplus}$ outer planet with a period of 5.6 days. They characterized the host star by applying the SpecMatch (Petigura 2015) and isochrones (Morton 2015a) packages to optical spectra acquired with Keck/HIRES. Using IRTF/ SpeX NIR spectra, we classified the host star as a K4 dwarf with a cooler temperature of $4402_{-93}^{+96} \mathrm{~K}$ and a smaller radius of $0.62 \pm 0.03$ (D17). After refitting the transit photometry, we revised the planet radii to $1.32 \pm 0.08 R_{\oplus}$ for the inner planet and $1.93_{-0.11}^{+0.13} R_{\oplus}$ for the outer planet. Both of these estimates are consistent with the Sinukoff et al. (2016) estimates.

\subsubsection{EPIC 206011691 (K2-21): Validated Two-planet System}

The NASA Exoplanet Archive describes the EPIC 206011691 system as containing a $1.25 \pm 0.11 R_{\oplus}$ inner planet on a 9.324 day orbit and a $1.53 \pm 0.14 R_{\oplus}$ outer planet with an orbital period of 15.498 days. Both planets were announced by Petigura et al. (2015), independently detected by Vanderburg et al. (2016b) and validated by Crossfield et al. (2016). In addition, Barros et al. (2016) detected the outer planet, but not the inner one.

The two planets are near the second-order 5:3 mean motion resonance, which may be a residual sign of past convergent migration. Neither planet is habitable and at $V=12.316$ the host star is a challenging target for precise planet mass measurements via RV, but the system might exhibit TTVs. Accordingly, the system is one of the targets of our ongoing 
Spitzer program to conduct follow-up transit observations of K2 planets (Program 10067, PI: M. Werner; Beichman et al. 2016).

Combining our new stellar characterization in D17 with our revised transit fits, we estimate the radii of the planets as $1.85 \pm 0.1 R_{\oplus}$ for the inner planet and $2.49_{-0.19}^{+0.17} R_{\oplus}$ for the outer planet. Our results are larger than the Petigura et al. (2015) estimates $\left(1.59 \pm 0.43 \quad R_{\oplus}\right.$ and $1.92 \pm 0.53 R_{\oplus}$, respectively), but they agree within the errors.

\subsubsection{EPIC 210508766 (K2-83): Validated Two-planet System}

The EPIC 210508766 system contains an M1 dwarf orbited by two super-Earths: a $1.71 \pm 0.10 R_{\oplus}$ planet on a 2.747 day orbit and a $2.11 \pm 0.12 R_{\oplus}$ planet on a longer 9.997 day orbit. Neither planet is habitable $\left(F_{p}=39 \pm 6 F_{\oplus}\right.$ and $F_{p}=6.9_{-1.0}^{+1.1} F_{\oplus}$, respectively) and the system is too faint for RV mass measurement $(K p=13.844, K s=10.765)$, but obtaining transmission spectra of both planets would be an interesting exercise in comparative planetology.

Both planets were previously validated by Crossfield et al. (2016). As discussed in Section 5.2, we confirm the validation of both planets as long as we account for the fact that planets in multi-planet systems are less likely to be false positives (Lissauer et al. 2012; Sinukoff et al. 2016; Vanderburg et al. 2016a).

\subsubsection{EPIC 210968143 (K2-90): Validated Two-planet System}

EPIC 210968143 is a K5 dwarf hosting two planets validated by Crossfield et al. (2016) and re-validated in this paper. The outer planet $\left(2.53_{-0.13}^{+0.14} R_{\oplus}\right)$ has a 13.734 day orbital period and the inner planet $\left(1.30_{-0.11}^{+0.13} R_{\oplus}\right)$ has a 2.901 day orbital period. The two planets are far from resonance and the host star is too faint at optical wavelengths for precise mass measurement $(K p=13.723)$, but the brighter IR magnitude of $K s=10.36$ renders the system amenable to atmospheric investigations. The inner planet EPIC 210968143.02 is highly irradiated $\left(F_{p}=81_{-11}^{+13} F_{\oplus}\right)$, but the outer planet EPIC 210968143.01 receives much less insolation $\left(F_{p}=10.2_{-1.4}^{+1.7} F_{\oplus}\right)$. A transmission spectrum of EPIC 210968143.01 might therefore help improve our understanding of less highly irradiated mini-Neptune atmospheres.

\subsubsection{EPIC 211305568: Candidate Two-planet System}

This M1V star was observed by K2 during Campaign 5. There are two K2OIs associated with the target, but neither is particularly compelling. The deeper transit-like events are attributed to 211305568.01 , a $2.0_{-0.3}^{+39.2} R_{\oplus} \mathrm{K} 2 \mathrm{OI}$ with an orbital period of 11.6 days and the shallower events to 211305568.02, a small $\left(0.7 \pm 0.10 R_{\oplus}\right) \mathrm{K} 2 \mathrm{OI}$ with an ultra-short orbital period of 0.2 days. We calculated a high FPP for 211305568.01 $(\mathrm{FPP}=55 \%-100 \%)$, but VESPA could not fit the putative transits of EPIC 211305568.02 and therefore did not return an FPP. We classify both K2OIs as planet candidates in this paper, but we urge readers to obtain additional follow-up observations to verify the veracity of these signals. The star is moderately faint at optical wavelengths $(K p=13.849)$, but easily observable at redder wavelengths $(K s=10.608)$.

\subsubsection{EPIC 211331236 (K2-117): Newly Validated Two-planet System}

This system contains two short-period super-Earths (1.96 $\pm 0.12 R_{\oplus}$ at 1.292 days, $2.03 \pm 0.13 R_{\oplus}$ at 5.444 days) orbiting an M3 dwarf with a temperature of $3842 \pm 82 \mathrm{~K}$ and a radius of $0.49 \pm 0.03 R_{\odot}$. The star is too faint for $\mathrm{RV}$ observations $(K p=13.905, K s=10.589)$, but the system is amenable to atmospheric characterization (see Figure 11).

\subsubsection{EPIC 211428897: Candidate Three-planet System}

The M2 dwarf EPIC 211428897 hosts three transiting Earth-sized planets with orbital periods of $1.611,2.218$, and 4.969 days and radii of $0.75 \pm 0.08 R_{\oplus}, 0.65 \pm 0.07 R_{\oplus}$, and $0.67 \pm 0.08 R_{\oplus}$, respectively. The inner two planet candidates are near the first-order 4:3 mean motion resonance and planet candidates .01 and .03 (1.611 days and 4.969 days) are near the second-order 3:1 mean motion resonance. Due to the small planet sizes, the anticipated RV semi-amplitudes of $0.2-0.3 \mathrm{~m} \mathrm{~s}^{-1}$ are too small to be detected by current spectrographs (and the $K p=13.2, K s=9.624$ host star is too faint at optical wavelengths for high-precision RV observations), but the masses of the planets could be estimated via TTVs. The VESPA FPPs for these planets would be low enough to merit validation in the absence of nearby stars, but the presence of a nearby star precludes the use of VESPA for this system and means that the planets are larger than our estimates would suggest. The nearby star is roughly 1 magnitude fainter than and approximately 1 "! 1 away from the target star. The correction factor for the planet radius estimates depends on which of the targets hosts the planets, but is likely to be on the order of a few (Ciardi et al. 2015, 2017; Furlan et al. 2017).

\subsection{Systems Orbiting Bright Stars}

\subsubsection{EPIC 210707130 (K2-85): Relatively Bright Star Hosting a} Validated Earth-sized Planet with an Ultra-short Orbital Period

As shown in Figure 10, EPIC 210707130.01 (K2-85b) is one of the most attractive RV targets in our sample. This ultrashort-period $1.37 \pm 0.11 R_{\oplus}$ planet orbits a moderately bright $(K p=12.099, K s=9.466) \mathrm{K} 5$ dwarf every 0.685 days and was validated by Crossfield et al. (2016). Using the massradius relation from Weiss \& Marcy (2014), we estimate a planet mass of $3.3 M_{\oplus}$, which is consistent with the expectation that highly irradiated small planets tend to have rocky compositions (Dressing et al. 2015). Given the estimated host star mass of $0.70 M_{\odot}$, the anticipated RV semi-amplitude is $3.0 \mathrm{~m} \mathrm{~s}^{-1}$.

\subsubsection{9 (K2-126): Moderately Bright Star Hosting a Newly Validated Super-Earth}

EPIC 212460519 is a moderately bright star $(K p=12.445$, $K s=9.712$ ) hosting a $1.84_{-0.10}^{+0.11} R_{\oplus}$ planet with a 7.4 day orbital period. The planet is too hot to be habitable $\left(F_{p}=35_{-6}^{+7} F_{\oplus}\right)$, but might be an interesting target for atmospheric characterization. Assuming $m_{p}=5.4 M_{\oplus}$ as predicted by the Weiss \& Marcy (2014) mass-radius relation, EPIC 212460519.01 is expected to induce an RV semiamplitude of $2.2 \mathrm{~m} \mathrm{~s}^{-1}$. 


\section{Conclusions}

Due to the dependence of planet radius estimates on host star characterization, our recent work improving the classification of cool dwarfs observed by K2 significantly affected the assumed properties of the associated planet candidates. In general, we found that the host star radii were underestimated by $8 \%-40 \%$ (D17), implying that the initial planet radius estimates were also undersized. In this paper, we investigated 79 candidate transit signals in the lightcurves of 74 low-mass $\mathrm{K} 2$ target stars and provided an updated catalog of planet properties. Our revisions to the system properties include both improved classifications of the host stars from D17 and our new transit fits. As part of the analysis, we also assessed the credibility of each planet candidate by using the VESPA framework developed by Morton $(2012,2015 b)$ to calculate the probability that each transit-like event was caused by an astrophysical false positive rather than a genuine planetary transit.

In total, we considered 79 putative transit events. We rejected six as false positives, validated 16 as bona fide planets, and classified 17 new planet candidates. We also upheld the previous classifications of two false positives and provided updated planet radius estimates for 16 planet candidates and 22 validated planets announced in earlier publications.

Our cool dwarf planet sample is dominated by small worlds: $55 \%$ (39) are smaller than $2 R_{\oplus}$ and $85 \%$ (60) are smaller than Neptune. Compared to the planets detected during the prime Kepler mission, our candidates tend to orbit brighter stars that are more amenable to follow-up observations. In particular, the thirteen small planets with radii of $1.5-3 R_{\oplus}$ and host stars brighter than $K s=11$ are likely targets for atmospheric characterization studies with Spitzer, $H S T$, and JWST. Furthermore, radial velocity observations may be feasible for the brightest systems, particularly given the advent of red spectrographs like CARMENES (Quirrenbach et al. 2010), the Habitable Zone Planet Finder (Mahadevan et al. 2010), the Infrared Doppler instrument (Tamura et al. 2012; Kotani et al. 2014), iSHELL (Rayner et al. 2012), and SpiROU (Delfosse et al. 2013; Artigau et al. 2014).

As the $\mathrm{K} 2$ mission continues, additional planets will be detected around stars across the ecliptic. We will continue to conduct follow-up observations for future targets and eagerly await the opportunity to characterize the best systems with the JWST. Beginning in 2018, we will expand our follow-up program to include planet candidates detected by the Transiting Exoplanet Survey Satellite (Ricker et al. 2014; Sullivan et al. 2015), which is scheduled to launch in 2018 March.

Many of our targets were provided by the $\mathrm{K} 2$ California Consortium (K2C2). We thank $\mathrm{K} 2 \mathrm{C} 2$ for sharing their candidate lists and vetting products. We are grateful to Tim Morton for making VESPA publicly available and to Jennifer Winters, Nic Scott, and Lea Hirsch for assisting with speckle imaging. We also acknowledge helpful conversations with Chas Beichman, Eric Gaidos, Jessie Christiansen, Michael Werner, and Arturo Martinez. We thank the anonymous referee for providing helpful suggestions to improve the paper.

This work was performed under contract with the Jet Propulsion Laboratory (JPL) funded by NASA through the Sagan Fellowship Program executed by the NASA Exoplanet Science Institute. A.V. is supported by the NSF Graduate
Research Fellowship, grant No. DGE 1144152. This publication was made possible through the support of a grant from the John Templeton Foundation. The opinions expressed here are those of the authors and do not necessarily reflect the views of the John Templeton Foundation. This paper includes data collected by the $\mathrm{K} 2$ mission, which is funded by the NASA Science Mission directorate. This research has made use of the NASA Exoplanet Archive, and the Exoplanet Follow-up Observation Program website, which are operated by the California Institute of Technology, under contract with the National Aeronautics and Space Administration under the Exoplanet Exploration Program. We appreciate the willingness of ExoFOP users to share their follow-up observations with the broader K2 community.

Our stellar characterization follow-up observations were obtained at the Infrared Telescope Facility, which is operated by the University of Hawaii under contract NNH14CK55B with the National Aeronautics and Space Administration and at Palomar Observatory. We thank the staff at both observatories and the Caltech Remote Observing Facilities staff for supporting us during our many observing runs. We are grateful to the IRTF and Caltech TACs for awarding us telescope time.

Our contrast curves were based on observations obtained at Gemini Observatory, Kitt Peak National Observatory via the NN-EXPLORE program time allocation at the WIYN telescope, and the W.M. Keck Observatory. Kitt Peak National Observatory, National Optical Astronomy Observatory, is operated by the Association of Universities for Research in Astronomy (AURA) under a cooperative agreement with the National Science Foundation. Gemini Observatory is operated by the Association of Universities for Research in Astronomy, Inc., under a cooperative agreement with the NSF on behalf of the Gemini partnership: the National Science Foundation (United States), the National Research Council (Canada), CONICYT (Chile), Ministerio de Ciencia, Tecnología e Innovación Productiva (Argentina), and Ministério da Ciência, Tecnologia e Inovação (Brazil). The W.M. Keck Observatory is operated as a scientific partnership among the California Institute of Technology, the University of California and the National Aeronautics and Space Administration. The Observatory was made possible by the generous financial support of the W.M. Keck Foundation.

The authors wish to recognize and acknowledge the very significant cultural role and reverence that the summit of Maunakea has always had within the indigenous Hawaiian community. We are most fortunate to have the opportunity to conduct observations from this mountain.

Facilities: Kepler, K2, IRTF (SpeX), Palomar:Hale (TripleSpec, PALM-3000/PHARO), Gemini-N (DSSI, NIRI), Gemini-S (DSSI), Keck:II (NIRC2), WIYN (DSSI).

\section{Appendix Vetting Plots}

As discussed in Section 5, we have posted transit fits and false positive assessments for all K2OIs on the ExoFOP-K2 website. Figures 12-14 provide an example set of vetting data products. The K2OIs featured are a newly validated planet (EPIC211680698.01 = K2-118b, Figure 12), a newly detected planet candidate (EPIC 212634172.01, Figure 13), and a newly rejected false positive (EPIC 212679798.01, Figure 14). 

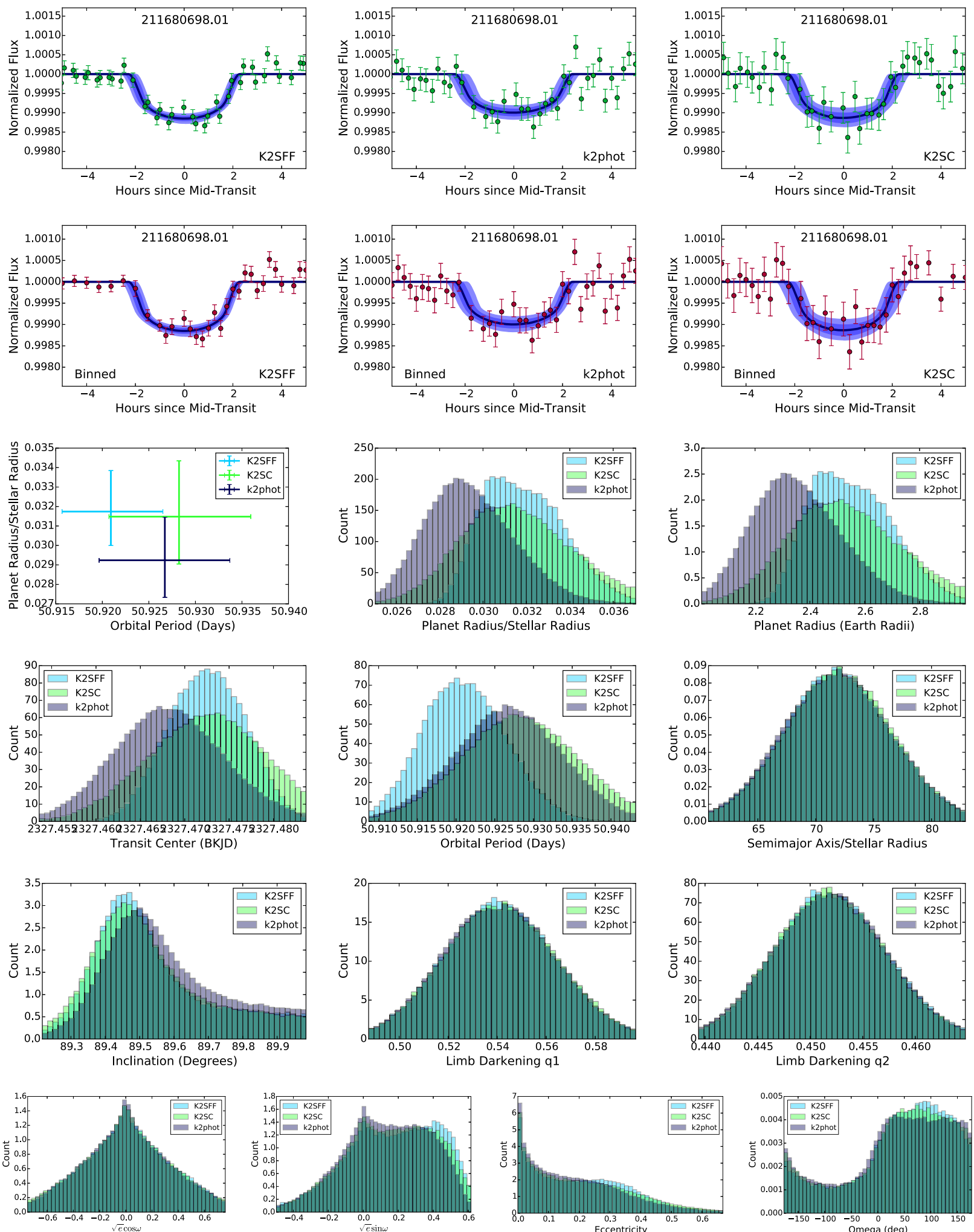

Figure 12. Example set of vetting plots showing transit fits and parameter posterior distributions for EPIC 211680698.01, a previously unknown planet validated in this paper as K2-118b. Top row: K2 data processed by the K2SFF (left), k2phot (middle), and K2SC (right) pipelines and phase-folded to the orbital period of EPIC 211680698.01. The dark blue lines indicate the median transit model fit to each version of the photometry and the medium (light) blue mark $1 \sigma(2 \sigma)$ contours. Second row: same as top row, but the K2 data points are binned to better reveal the quality of the transit fits. Third row, left column: comparison of the planet/star radius ratios and orbital periods found when fitting K2SFF (light blue), K2SC (green), and k2phot (dark purple) photometry. All remaining panels: posterior probability distributions for the indicated transit parameter. The translucent histograms compare the results produced by fitting K2 photometry processed using K2SFF (light blue), K2SC (light green), and k2phot (gray). 

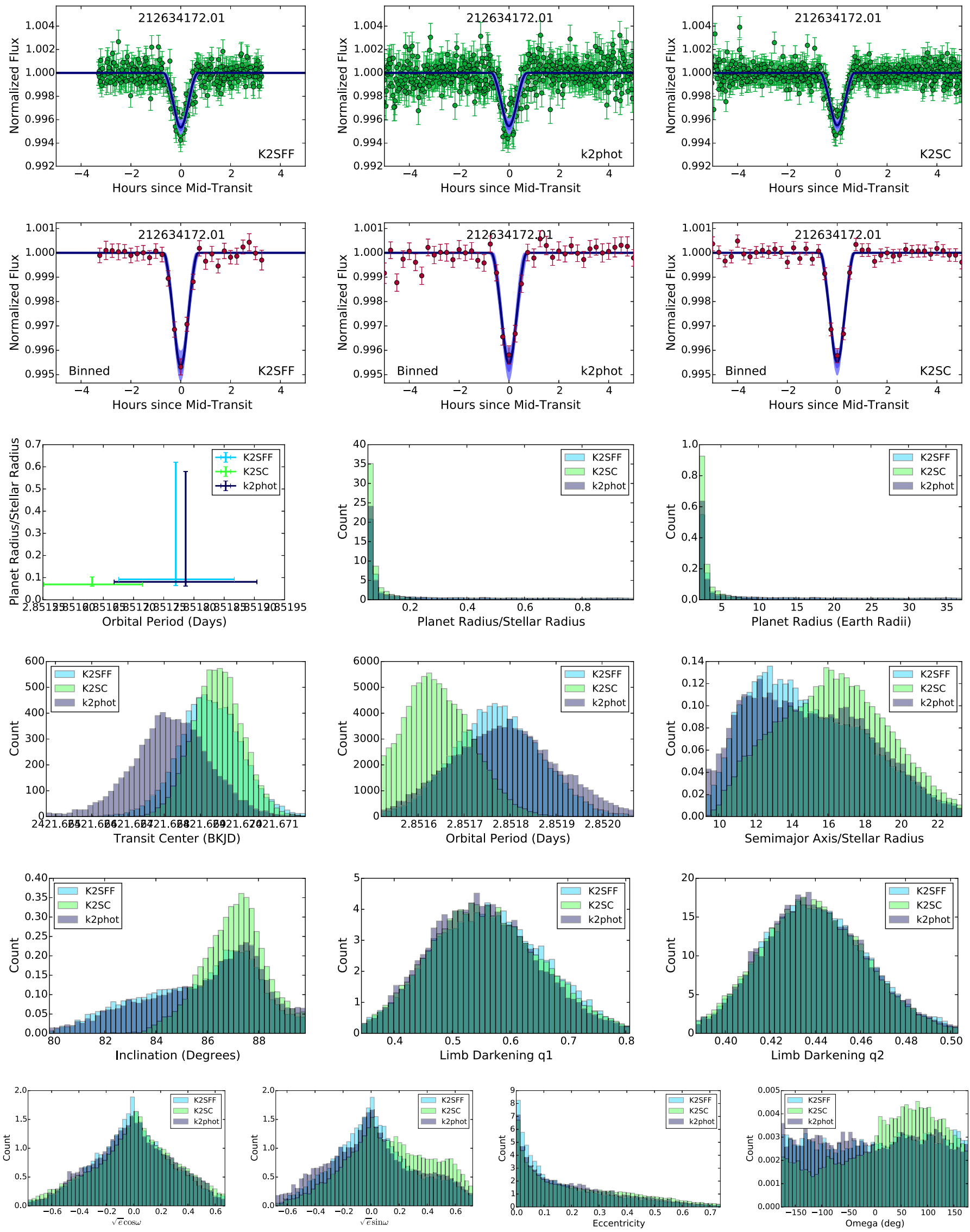

Figure 13. Same as Figure 12 but for the newly detected planet candidate EPIC 212634172.01. 

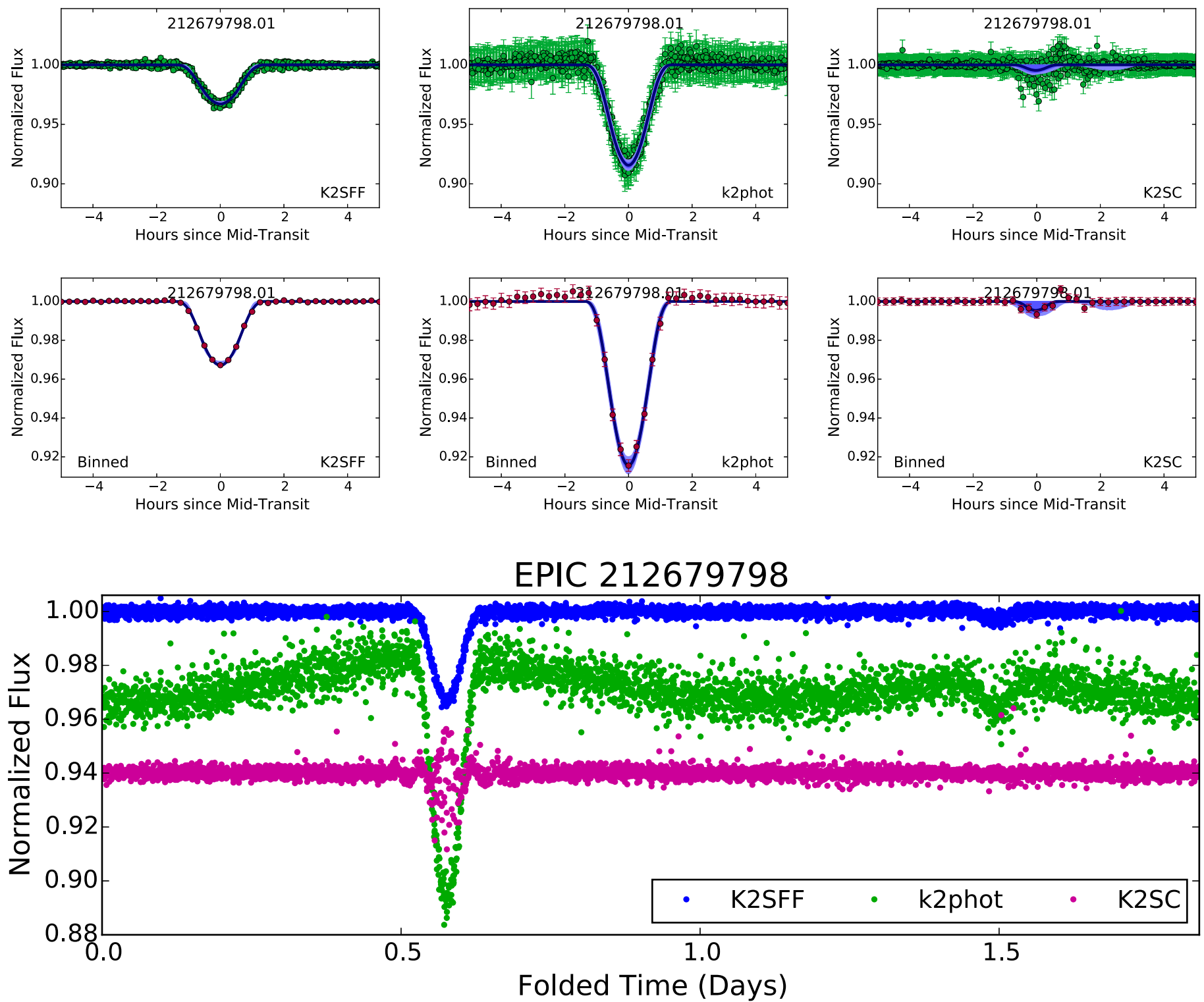

Figure 14. Top two rows: same as Figure 12 but for newly rejected false positive EPIC 212679798.01. Bottom row: phase-folded light curve of EPIC 212679798 shown over the full orbital period of K2OI 212679798.01. The KSFF (blue, top) and k2phot (green, middle) photometry display a clear secondary eclipse near 1.5 days. The $\mathrm{K} 2 \mathrm{SC}$ photometry (magenta, bottom) has removed the secondary eclipse and partially flattened the primary eclipse. Note that the depth of the primary and secondary eclipses are also deeper in the k2phot photometry than in the K2SFF photometry. The event depth discrepancy is likely due to the different default photometric apertures used by both pipelines.

\section{ORCID iDs}

Courtney D. Dressing (i) https://orcid.org/0000-00018189-0233

Andrew Vanderburg $(1)$ https://orcid.org/0000-00017246-5438

Joshua E. Schlieder (10 https://orcid.org/0000-0001-5347-7062

Elisabeth R. Newton (i) https://orcid.org/0000-0003-

4150-841X

Benjamin J. Fulton (1) https://orcid.org/0000-0003-3504-5316

Andrew W. Howard (1) https://orcid.org/0000-0001-

8638-0320

Howard Isaacson (1) https://orcid.org/0000-0002-0531-1073

Erik A. Petigura (ํ) https://orcid.org/0000-0003-0967-2893

Evan Sinukoff 10 https://orcid.org/0000-0002-5658-0601

Mark Everett (i) https://orcid.org/0000-0002-0885-7215
Elliott Horch (1) https://orcid.org/0000-0003-2159-1463

Steve B. Howell (1) https://orcid.org/0000-0002-2532-2853

\section{References}

Adams, E. R., Jackson, B., \& Endl, M. 2016, arXiv:1603.06488 Aigrain, S., Hodgkin, S. T., Irwin, M. J., Lewis, J. R., \& Roberts, S. J. 2015 , MNRAS, 447, 2880

Aigrain, S., Parviainen, H., \& Pope, B. J. S. 2016, MNRAS, 459, 2408

Akeson, R. L., Chen, X., Ciardi, D., et al. 2013, PASP, 125, 989

Armstrong, D. J., Kirk, J., Lam, K. W. F., et al. 2015, A\&A, 579, A19

Armstrong, D. J., Kirk, J., Lam, K. W. F., et al. 2016, MNRAS, 456, 2260

Armstrong, D. J., Osborn, H. P., Brown, D. J. A., et al. 2014, arXiv:1411.6830

Artigau, E, Kouach, D., Donati, J.-F., et al. 2014, Proc. SPIE, 9147, 15

Baraffe, I., Selsis, F., Chabrier, G., et al. 2004, A\&A, 419, L13

Barros, S. C. C., Demangeon, O., \& Deleuil, M. 2016, arXiv:1607.02339

Beichman, C., Livingston, J., Werner, M., et al. 2016, ApJ, 822, 39

Boyajian, T. S., von Braun, K., van Belle, G., et al. 2012, ApJ, 757, 112 
Boyajian, T. S., von Braun, K., van Belle, G., et al. 2013, ApJ, 771, 40 Casagrande, L., Flynn, C., \& Bessell, M. 2008, MNRAS, 389, 585

Chen, J., \& Kipping, D. 2017, ApJ, 834, 17

Ciardi, D. R., Beichman, C. A., Horch, E. P., \& Howell, S. B. 2015, ApJ, 805,16

Ciardi, D. R., Beichman, C. A., Horch, E. P., \& Howell, S. B. 2017, ApJ, 834, 96

Claret, A., Hauschildt, P. H., \& Witte, S. 2012, A\&A, 546, A14

Crossfield, I. J. M., Ciardi, D. R., Petigura, E., et al. 2016, ApJS, 226, 7

Delfosse, X., Bonfils, X., Forveille, T., et al. 2013, A\&A, 553, A8

Díaz, R. F., Almenara, J. M., Santerne, A., et al. 2014, MNRAS, 441, 983

Dressing, C. D., \& Charbonneau, D. 2013, ApJ, 767, 95

Dressing, C. D., Charbonneau, D., Dumusque, X., et al. 2015, ApJ, 800, 135

Dressing, C. D., Newton, E. R., Schlieder, J. E., et al. 2017, ApJ, 836, 167

Eastman, J., Gaudi, B. S., \& Agol, E. 2013, PASP, 125, 83

Feiden, G. A., \& Chaboyer, B. 2012, ApJ, 757, 42

Foreman-Mackey, D., Hogg, D. W., Lang, D., \& Goodman, J. 2013, PASP, 125,306

Foreman-Mackey, D., Montet, B. T., Hogg, D. W., et al. 2015, ApJ, 806, 215

Fressin, F., Torres, G., Charbonneau, D., et al. 2013, ApJ, 766, 81

Fressin, F., Torres, G., Désert, J.-M., et al. 2011, ApJS, 197, 5

Furlan, E., Ciardi, D. R., Everett, M. E., et al. 2017, AJ, 153, 71

González Hernández, J. I., \& Bonifacio, P. 2009, A\&A, 497, 497

Hayward, T. L., Brandl, B., Pirger, B., et al. 2001, PASP, 113, 105

Hirano, T., Fukui, A., Mann, A. W., et al. 2016, ApJ, 820, 41

Hodapp, K. W., Jensen, J. B., Irwin, E. M., et al. 2003, PASP, 115, 1388

Horch, E. P., Howell, S. B., Everett, M. E., \& Ciardi, D. R. 2012, AJ, 144, 165

Howell, S. B., Everett, M. E., Sherry, W., Horch, E., \& Ciardi, D. R. 2011, AJ, 142,19

Howell, S. B., Sobeck, C., Haas, M., et al. 2014, PASP, 126, 398

Huber, D., Bryson, S. T., Haas, M. R., et al. 2016, ApJS, 224, 2

Huber, D., Silva Aguirre, V., Matthews, J. M., et al. 2014, ApJS, 211, 2

Kipping, D. M. 2013a, MNRAS, 435, 2152

Kipping, D. M. 2013b, MNRAS, 434, L51

Kopparapu, R. K., Ramirez, R., Kasting, J. F., et al. 2013, ApJ, 765, 131

Kopparapu, R. K., Ramirez, R. M., SchottelKotte, J., et al. 2014, ApJL, 787, L29

Kotani, T., Tamura, M., Suto, H., et al. 2014, Proc. SPIE, 9147, 14

Kraus, A. L., Tucker, R. A., Thompson, M. I., Craine, E. R., \& Hillenbrand, L. A. 2011, ApJ, 728, 48

Kreidberg, L. 2015, PASP, 127, 1161

Kurokawa, H., \& Kaltenegger, L. 2013, MNRAS, 433, 3239

Lammer, H., Selsis, F., Ribas, I., et al. 2003, ApJL, 598, L121

Lissauer, J. J., Marcy, G. W., Rowe, J. F., et al. 2012, ApJ, 750, 112

Lopez, E. D., \& Fortney, J. J. 2013, ApJ, 776, 2

Lopez, E. D., Fortney, J. J., \& Miller, N. 2012, ApJ, 761, 59

Luger, R., Agol, E., Kruse, E., et al. 2016, AJ, 152, 100

Mahadevan, S., Ramsey, L., Wright, J., et al. 2010, Proc. SPIE, 7735, 77356X

Mandel, K., \& Agol, E. 2002, ApJL, 580, L171
Mann, A. W., Brewer, J. M., Gaidos, E., Lépine, S., \& Hilton, E. J. 2013a, AJ, 145,52

Mann, A. W., Feiden, G. A., Gaidos, E., Boyajian, T., \& von Braun, K. 2015 , ApJ, 804, 64

Mann, A. W., Gaidos, E., \& Ansdell, M. 2013b, ApJ, 779, 188

Mann, A. W., Gaidos, E., Vanderburg, A., et al. 2017, AJ, 153, 64

Martinez, A. O., Crossfield, I. J. M., Schlieder, J. E., et al. 2017, ApJ, 837, 72

Montet, B. T., Morton, T. D., Foreman-Mackey, D., et al. 2015, ApJ, 809, 25

Morton, T. D. 2012, ApJ, 761, 6

Morton, T. D. 2015a, Isochrones: Stellar Model Grid Package, Astrophysics Source Code Library, ascl:1503.010

Morton, T. D. 2015b, VESPA: False positive probabilities calculator, Astrophysics Source Code Library, ascl:1503.011

Muirhead, P. S., Becker, J., Feiden, G. A., et al. 2014, ApJS, 213, 5

Muirhead, P. S., Hamren, K., Schlawin, E., et al. 2012, ApJL, 750, L37

Murray-Clay, R. A., Chiang, E. I., \& Murray, N. 2009, ApJ, 693, 23

Newton, E. R., Charbonneau, D., Irwin, J., \& Mann, A. W. 2015, ApJ, 800, 85

Owen, J. E., \& Wu, Y. 2013, ApJ, 775, 105

Pecaut, M. J., \& Mamajek, E. E. 2013, ApJS, 208, 9

Petigura, E. A. 2015, PhD thesis, Univ. California

Petigura, E. A., Marcy, G. W., \& Howard, A. W. 2013, ApJ, 770, 69

Petigura, E. A., Schlieder, J. E., Crossfield, I. J. M., et al. 2015, ApJ, 811, 102

Pope, B. J. S., Parviainen, H., \& Aigrain, S. 2016, MNRAS, arXiv:1606.01264

Quirrenbach, A., Amado, P. J., Mandel, H., et al. 2010, Proc. SPIE, 7735, 773513

Rayner, J., Bond, T., Bonnet, M., et al. 2012, Proc. SPIE, 8446, 84462C

Ricker, G. R., Winn, J. N., Vanderspek, R., et al. 2014, JATIS, 1, 014003

Sanchis-Ojeda, R., Rappaport, S., Pallè, E., et al. 2015, ApJ, 812, 112

Sanchis-Ojeda, R., Rappaport, S., Winn, J. N., et al. 2014, ApJ, 787, 47

Sanz-Forcada, J., Micela, G., Ribas, I., et al. 2011, A\&A, 532, A6

Schmitt, J. R., Tokovinin, A., Wang, J., et al. 2016, AJ, 151, 159

Seager, S., \& Mallén-Ornelas, G. 2003, ApJ, 585, 1038

Sinukoff, E., Howard, A. W., Petigura, E. A., et al. 2016, ApJ, 827, 78

Sozzetti, A., Torres, G., Charbonneau, D., et al. 2007, ApJ, 664, 1190

Spada, F., Demarque, P., Kim, Y.-C., \& Sills, A. 2013, ApJ, 776, 87

Sullivan, P. W., Winn, J. N., Berta-Thompson, Z. K., et al. 2015, ApJ, 809, 77

Tamura, M., Suto, H., Nishikawa, J., et al. 2012, Proc. SPIE, 8446, 84461 T

Torres, G., Fressin, F., Batalha, N. M., et al. 2011, ApJ, 727, 24

Torres, G., Winn, J. N., \& Holman, M. J. 2008, ApJ, 677, 1324

Valencia, D., Ikoma, M., Guillot, T., \& Nettelmann, N. 2010, A\&A, 516, A20

Van Cleve, J. E., Howell, S. B., Smith, J. C., et al. 2016, PASP, 128, 075002

Vanderburg, A., Bieryla, A., Duev, D. A., et al. 2016a, ApJL, 829, L9

Vanderburg, A., \& Johnson, J. A. 2014, PASP, 126, 948

Vanderburg, A., Latham, D. W., Buchhave, L. A., et al. 2016b, ApJS, 222, 14

von Braun, K., Boyajian, T. S., van Belle, G. T., et al. 2014, MNRAS, 438, 2413

Watson, A. J., Donahue, T. M., \& Walker, J. C. G. 1981, Icar, 48, 150

Weiss, L. M., \& Marcy, G. W. 2014, ApJL, 783, L6

Wolfgang, A., \& Lopez, E. 2015, ApJ, 806, 183 